\title{
Tilings of non-convex Polygons, skew-Young Tableaux and determinantal Processes
}

\author{
Mark Adler* Kurt Johansson ${ }^{\dagger} \quad$ Pierre van Moerbeke ${ }^{\ddagger}$
}

\section{Contents}

1 Introduction and main results 2

2 Interlacing and measures on skew-Young Tableaux 12

3 An alternative integral representation for $h_{y-y_{j}}\left(1^{n}\right)$ and some determinantal identities

4 From Karlin-McGregor to the $\mathbb{K}_{q}$-kernel 26

5 Transforming $M$ into $\tilde{M}$ and computing its inverse 36

6 The integral representation of the $\mathbb{K}_{q}$-kernel 40

7 The $\mathbb{K}^{\text {red }}$-kernel as a limit of the $\mathbb{K}_{q}$-kernel, for $q \rightarrow 1$

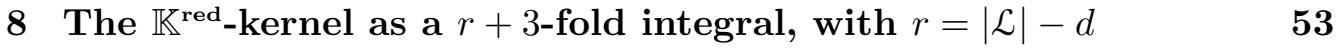

*2000 Mathematics Subject Classification. Primary: 60G60, 60G65, 35Q53; secondary: 60G10, 35Q58. Key words and Phrases:Lozenge tilings, non-convex polygons, kernels. Department of Mathematics, Brandeis University, Waltham, Mass 02453, USA. E-mail: adler@brandeis.edu. The support of a Simons Foundation Grant \# 278931 is gratefully acknowledged. M.A. thanks the Simons Center for Geometry and Physics for its hospitality.

${ }^{\dagger}$ Department of Mathematics, KTH Royal Institute of Technology, Stockholm, Sweden. Email: kurtj@kth.se. The support of the Swedish Research Council (VR) and grant KAW 2010.0063 of the Knut and Alice Wallenberg Foundation are gratefully acknowledged.

${ }^{\ddagger}$ Department of Mathematics, Université de Louvain, 1348 Louvain-la-Neuve, Belgium and Brandeis University, Waltham, Mass 02453, USA. E-mail: pierre.vanmoerbeke@uclouvain.be . The support of a Simons Foundation Grant \# 280945 is gratefully acknowledged. PvM thanks the Simons Center for Geometry and Physics, Stony Brook, and the Kavli Institute of Physics, Santa Barbara, for their hospitality. 


\begin{abstract}
This paper studies random lozenge tilings of general non-convex polygonal regions. We show that the pairwise interaction of the non-convexities leads asymptotically to new kernels and thus to new statistics for the tiling fluctuations. The precise geometrical figure here consists of a hexagon with cuts along opposite edges. For this model we take limits when the size of the hexagon and the cuts tend to infinity, while keeping certain geometric data fixed in order to guarantee interaction beyond the limit. We show in this paper that the kernel for the finite tiling model can be expressed as a multiple integral, where the number of integrations is related to the fixed geometric data above. The limiting kernel is believed to be a universal master kernel.
\end{abstract}

\title{
1 Introduction and main results
}

The purpose of this paper is to study random lozenge tilings of non-convex polygonal regions. Non-convex figures are particularly interesting due to the appearance of new statistics for the tiling fluctuations, caused by the non-convexities themselves or by the interaction of these non-convexities. The final goal will be to study the asymptotics of the tiling statistical fluctuations in the neighborhood of these non-convexities, when the polygons tend to an appropriate scaling limit.

The tiling problems of hexagons by lozenges goes back to the celebrated 1911formula on the enumeration of lozenge tilings of hexagons of sides $a, b, c, a, b, c$ by the Scottish mathematician MacMahon [32. This result has been extended in the combinatorics community to many different shapes, including non-convex domains, in particular to shapes with cuts and holes; see e.g., Ciucu, Fischer and Krattenthaler [11, 30].

Tiling problems have been linked to Gelfand-Zetlin cones by Cohn, Larsen, Propp [12, and to non-intersecting paths, determinantal processes, kernels and random matrices by Johansson [17, 18, 19]. In [20], Johansson showed that the statistics of the lozenge tilings of hexagons was governed by a kernel consisting of discrete Hahn polynomials; see also Gorin 24]. In [21] and [23], it was shown that, in appropriate limits, the tiles near the boundary between the frozen and stochastic region (arctic circle) fluctuate according to the Airy process and near the points of tangency of the arctic circle with the edge as the GUE-minor process.

Tiling of non-convex domains were investigated by Okounkov-Reshetikhin [36] and Kenyon-Okounkov [28] from a macroscopic point of view. Further important phenomena for nonconvex domains appear in the work of Borodin, Gorin and Rains [8], Defosseux [13], Metcalfe [33, Petrov [37, 38], Gorin [25], Novak [34, Bufetov and Knizel [6], Duse and Metcalfe [15, 16], and Duse, Johansson and Metcalfe [14]; see also the recent paper by Betea, Bouttier, Nejjar and Vuletic [5].

The present study consisting of two papers leads to the so-called Discrete Tacnode Kernel (13), which we believe to be a master kernel, from which many other kernels can be deduced (see Fig.1); namely,

(1) the GUE-tacnode kernel for overlapping Aztec diamonds [2, 1, 4] (also a nonconvex geometry), when the size of the overlap remains small compared to the 
size of the diamonds. See also coupled GUE-matrices [4].

(2) the Tacnode kernel in the context of colliding Brownian motions and double Aztec diamonds.

(3) The cusp-Airy kernel ([14]) should also be a scaling limit of the Discrete Tacnode Kernel (13), etc...

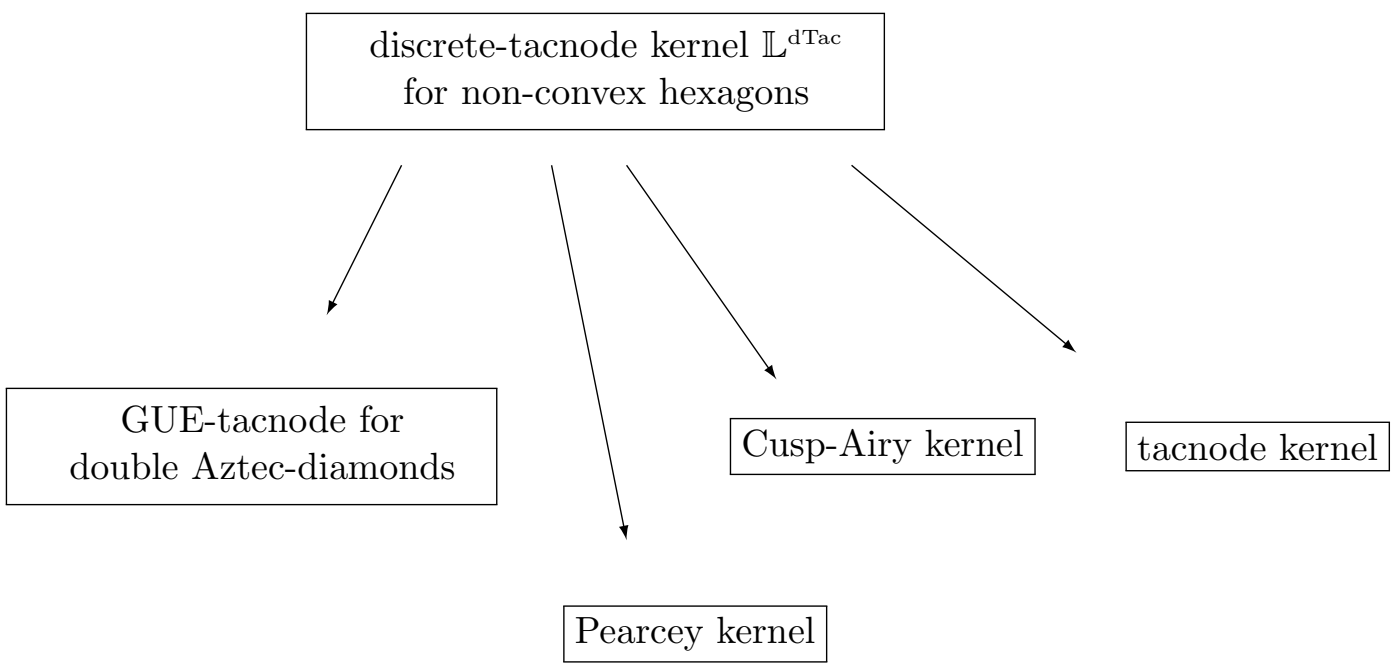

Fig.1. Is the statistics associated with the discrete-tacnode kernel for nonconvex hexagons universal ? Does it imply in some appropriate limit all these known statistics? Is it a master-kernel?

This led us to investigate determinantal processes for lozenge tilings of fairly general non-convex polygons, with non-convexities facing each other. This is going much beyond Petrov's work [38 on the subject, and yet inspired by some of his techniques. Consider a hexagon with several cuts as in Fig.2, and a tiling with lozenges of the shape as in Fig.3, colored blue, red and green; introducing a cut amounts to covering a region with red tiles. Note there is an affine transformation from our tiles to the usual ones in the literature; see e.g. the simulation of Fig.5. The right-leaning blue tiles turn into our blue ones, the up-right red ones into our red ones and the left-leaning green tiles $\left(30^{\circ}\right)$ to our green tiles $\left(45^{\circ}\right)$, all as in Fig.2.

Two different determinantal pocesses, a $\mathbb{K}$-process and an $\mathbb{L}$-process, will be considered, depending on the angle at which one looks at the polygons; south to north for the $\mathbb{K}$-process or south-west to north-east for the $\mathbb{L}$-process. In this series of two papers, the first one will focus on the $\mathbb{K}$-process and its kernel, and the second one [3] on the $\mathbb{L}$-process, its kernel and its asymptotic limit in between the non-convexities. Nevertheless both processes will be introduced in this paper. 


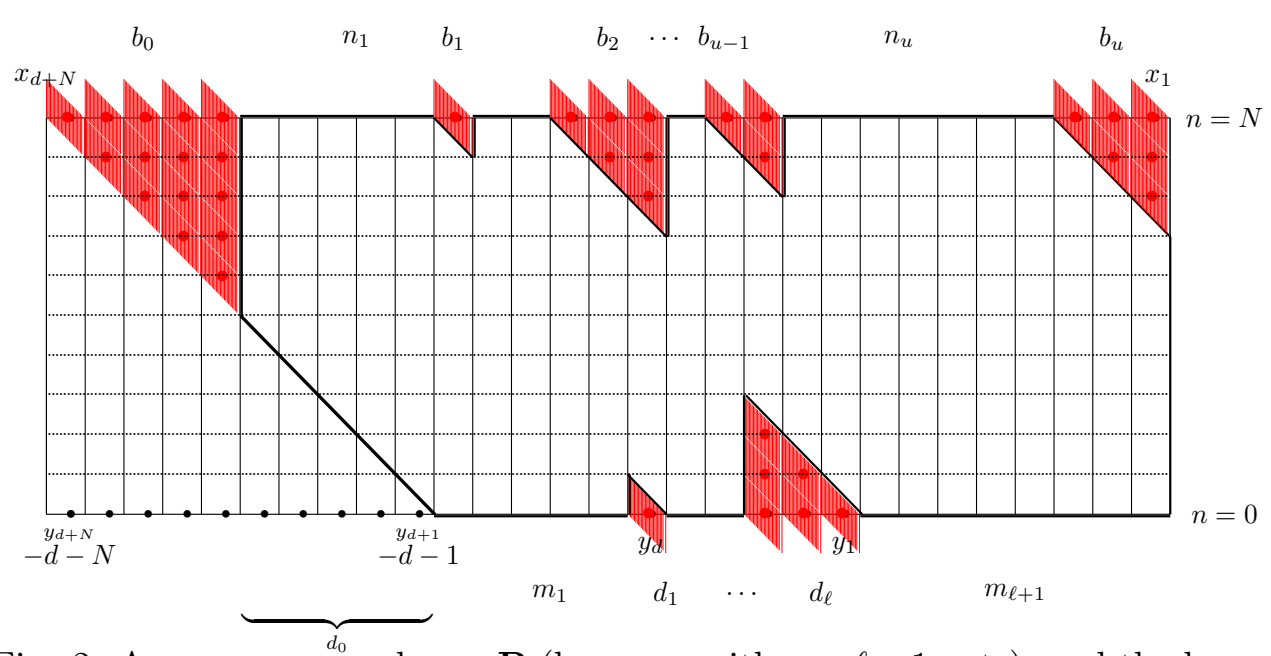

Fig. 2: A non-convex polygon $\mathbf{P}$ (hexagon with $u+\ell-1$ cuts), and the hexagon $\widetilde{\mathbf{P}}=\mathbf{P} \cup\{u+\ell+1$ red triangles $\}$. (multicut model)

A good part of the work will consist of reducing the number of integrations in the $\mathbb{K}$-kernel to $r+3$, where $r$, an integer defined in (5), relates to the geometry of the polygon and of the $\mathbb{L}$-process. In the second paper, the $\mathbb{L}$-kernel will require many more transformations in order to be in the right shape to perform asymptotics. As a preview of the second paper [3] we merely state here the form of the $\mathbb{L}$-kernel and the asymptotics without proof. Incidentally, the $\mathbb{K}$-kernel should also lead to interesting open questions related to the Gaussian Free Field (Petrov [37]) and also to open questions related to Petrov's [37] and Gorin's [25] work; see comments after Theorem 1.1 .

To be precise, and as shown in Figure 2, we consider a general non-convex polygonal region $\mathbf{P}$ (multicut model) consisting of taking a hexagon where two opposite edges have cuts, $u-1$ cuts $b_{1}, b_{2}, \ldots, b_{u-1}$ cut out of the upper-part and $\ell$ cuts $d_{1}, d_{2}, \ldots, d_{\ell}$ cut out of the lower-part 1 let $d:=\sum_{1}^{\ell} d_{i}$. Let $b_{0}$ and $b_{u}$ be the "cuts" corresponding to the two triangles added to the left and the right of $\mathbf{P}$ and let $d_{0}$ be the size of the lower-oblique side. Then $N:=b_{0}+d_{0}$ is the distance between the lower and upper edges. The intervals separating the upper-cuts (resp. lower-cuts) are denoted by $n_{i}$ (resp. $m_{i}$ ) and we require them to satisfy $\sum_{1}^{\ell+1} m_{i}=\sum_{1}^{u} n_{i}$, which is equivalent to $\sum_{0}^{u} b_{i}=d+N$. Define $\widetilde{\mathbf{P}}$ to be the quadrilateral (with two parallel sides) obtained by adding the red triangles to $\mathbf{P}$, as in Fig.1.

Introduce the coordinates $(n, x) \in \mathbb{Z}^{2}$, where $n=0$ and $n=N$ refer to the lower and upper sides of the polygon, with $x$ being the running variable along the lines $n$. The vertices of $\mathbf{P}$ and $\widetilde{\mathbf{P}}$ all belong to the vertical lines $x=\{$ half-integers $\}$ of the grid (in Fig.2). The $d=\sum_{1}^{\ell} d_{i}$ integer points in $\{\widetilde{\mathbf{P}} \backslash \mathbf{P}\} \cap\{n=0\}$ are labeled by $y_{1}>\ldots>y_{d}$; they are the integers in the cuts. We complete that set with the integer points to the left of $\widetilde{\mathbf{P}}$ along $\{n=0\}$; they are labeled by $y_{d+1}>\cdots>y_{d+N}$ and we set $y_{d+1}=-d-1$ and $y_{d+N}=-d-N$. This fixes the origin of the $x$-coordinate. Similarly, the integer points $\{\widetilde{\mathbf{P}} \backslash \mathbf{P}\} \cap\{n=N\}$

\footnotetext{
${ }^{1}$ The $b_{i}$ and $d_{i}$ 's also denote the size of the cuts.
} 
are labeled by $x_{1}>\cdots>x_{d+N}=-d-N$. We assume that $x_{i} \geq y_{i}$ for all $1 \leq i \leq d+N$, and that $y_{d} \notin\{x$-coordinates of an upper-cut $\}$.

Besides the $(n, x)$-coordinates, another set of coordinates $(\eta, \xi)$ will also be convenient (see Fig. 3):

$$
\eta=n+x+\frac{1}{2}, \quad \xi=n-x-\frac{1}{2} \quad \Leftrightarrow \quad n=\frac{1}{2}(\eta+\xi), \quad x=\frac{1}{2}(\eta-\xi-1) .
$$

Assuming $y_{1}-y_{d} \leq N-1$, we define polynomials 2 ,

$$
P(z):=\left(z-y_{d}+1\right)_{N-\left(y_{1}-y_{d}+1\right)} \text { and } Q(z):=\prod_{1}^{d+N}\left(z-x_{i}\right) .
$$

The roots $x_{i}$ of $Q(z)$, compared to the roots $y_{1}-N+1<\cdots<y_{d}-1$ of $P(z)$, can be subdivided into three sets, the $\mathcal{L}$ (eft), the $\mathcal{R}$ (ight), the $\mathcal{C}$ (enter), and a set $\mathcal{G}(\mathrm{ap})$ not containing any $x_{i}$ :

$$
\begin{aligned}
\mathcal{L} & :=\left\{x_{i}, \text { such that } x_{i}<y_{1}-N+1\right\} \\
\mathcal{R} & :=\left\{x_{i}, \text { such that } x_{i} \geq y_{d}\right\} \\
\mathcal{C} & :=\left\{x_{1}, \ldots, x_{d+N}\right\} \backslash(\mathcal{L} \cup \mathcal{R}) \\
\mathcal{G} & :=\left\{y_{1}-N+1, \ldots, y_{d}-1\right\} \backslash \mathcal{C},
\end{aligned}
$$

ensuing the decompositions in polynomials

$$
P(z)=Q_{\mathcal{C}}(z) P_{\mathcal{G}}(z) \text {, and } \quad Q(z)=Q_{\mathcal{L}}(z) Q_{\mathcal{C}}(z) Q_{\mathcal{R}}(z) .
$$

The number $r$, assumed positive, will play an important role:

$$
r:=|\mathcal{L}|-d
$$

Referring to contour integration in this paper, the notation $\Gamma$ (set of points) will denote a contour encompassing the points in question and no other poles of the integrands; e.g., contours like

$$
\Gamma(\mathcal{R}), \Gamma(\mathcal{L}), \Gamma(x+\mathbb{N}), \ldots
$$

The $\mathbb{K}$ and $\mathbb{L}$-processes. Given a covering of this polygonal shape with tiles of three shapes, colored in red, blue and green tiles, as in Fig.2, put a red and blue dot in the middle of the red and blue tiles. The red dots belong to the intersections of the vertical lines $x=$ integers and the horizontal lines $n=0, \ldots, N$; they define a point process $(n, x)$, which we call the $\mathbb{K}^{\text {red }}$-process. The initial condition at the bottom $n=0$ is given by the $d$ fixed red dots at integer locations in the lower-cuts, whereas the final condition at the top $n=N$ is given by the $d+N$ fixed red dots in the upper-cuts, including the red dots to

\footnotetext{
${ }^{2}$ For any integers $k \in \mathbb{Z}$ and $N \geq 0$ we have $k_{0}=1$ and $k_{N}=k(k+1) \ldots(k+N-1)$.
} 
the left and to the right of the figure, all at integer locations. Notice that the process of red dots on $\widetilde{\mathbf{P}}$ form an interlacing set of integers starting from $d$ fixed dots (contiguous for the two-cut and non-contiguous for the multi-cut model) and growing linearly to end up with a set of $d+N$ (non-contiguous) fixed dots. This can be viewed as a "truncated" Gel'fand-Zetlin cone!

The blue dots belong to the intersection $\in \mathbf{P}$ of the parallel oblique lines $n+x=k-\frac{1}{2}$ with the horizontal lines $n=\ell-\frac{1}{2}$ for $k, \ell \in \mathbb{Z}$; in terms of the coordinates (1), the blue dots are parametrized by $(\eta, \xi)=(k, 2 \ell-k-1) \in \mathbb{Z}^{2}$, with $(k, \ell)$ as above. It follows that the $(\eta, \xi)$-coordinates of the blue dots satisfy $\eta+\xi=1,3, \ldots, 2 N-1$. This point process defines the $\mathbb{L}^{\text {blue }}$-process. The blue dots on the oblique lines also interlace, going from left to right, but their numbers go up, down, up and down again. The number of blue dots per oblique line $n+x=k-\frac{1}{2}$ is given by the difference between the heights computed at the points $n=0$ and $n=N$ along that line; see Fig.7. A special feature appears in the two-cut model.

One could also consider an $\mathbb{L}^{\text {green}}$-process, by putting a green dot on the green tiles. It would lead to a a determinantal process of green particles on the vertical lines $x=$ half integers, where the number $r$ in (5) would be replaced by $s=|\mathcal{R}|-d$; see Fig.4. In the end, this kernel would be similar to the $\mathbb{L}^{\text {blue }}$-kernel.

As it turns out, the two kernels $\mathbb{K}^{\text {red }}$ and $\mathbb{L}^{\text {blue }}$ are highly related, as will be shown in [3], where also the asymptotics for the $\mathbb{L}$-kernel will be carried out. Indeed, both point processes can be described by dimers on the points of the associated bipartite graph dual to $\mathbf{P}$; the two kernels are related by the inverse Kasteleyn matrix of the bipartite graph. The aim of this first paper is to find a

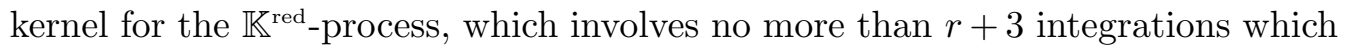
is a conditio sine qua non for taking asymptotics for $\mathbb{L}^{\text {blue }}$ while keeping $r$ fixed.

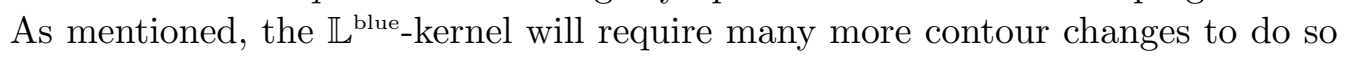
in [3]. This kernel could also be used for showing the Gaussian Free Field result in the bulk, again keeping $r$ fixed.

The following rational function,

$$
h(u):=\frac{Q_{\mathcal{R}}(u)}{P_{\mathcal{G}}(u) Q_{\mathcal{L}}(u)},
$$

appears crucially in the following $k$-fold contour integra $t^{3}$ for $k \geq 0$, (see $(6)$ )

$$
\Omega_{k}^{ \pm}(v, z):=\left(\prod_{\alpha=1}^{k} \oint_{\Gamma(\mathcal{L})} \frac{d u_{\alpha} h\left(u_{\alpha}\right)\left(z-u_{\alpha}\right)^{ \pm 1}}{2 \pi \mathrm{i}\left(v-u_{\alpha}\right)}\right) \Delta_{k}^{2}(u)\left\{\begin{array}{l}
\left.\widetilde{E}_{g}^{\left(\mathbf{y}_{\text {cut }}\right)}\right)\left(u_{1}, \ldots, u_{k}\right) \\
\widetilde{E}_{g}^{\left(\mathbf{y}_{\text {cut }}\right)}\left(z ; u_{1}, \ldots, u_{k}\right)
\end{array} .\right.
$$

\footnotetext{
${ }^{3}$ Set $\Omega_{0}^{+}(v, z)=1$. A shorthand notation for the Vandermonde is $\Delta_{k}(u):=\Delta_{k}\left(u_{1}, \ldots, u_{k}\right)=$ $\prod_{1 \leq i<j \leq k}\left(u_{i}-u_{j}\right)$.
} 
where $\widetilde{E}_{g}^{\left(\mathbf{y}_{\text {cut }}\right)}$ is a symmetric function of the variables $u_{1}, \ldots, u_{k}$, which depend on the integer points $y_{d}<\cdots<y_{1}$ in the lower-cuts, with $g=y_{1}-y_{d}-d+1=$ the number of gaps in that sequence. The precise symmetric function will be given later in (42) and (44). When that sequence is contiguous, we have $g=0$ and the symmetric function equals 1; e.g., this is so when $\mathbf{P}$ has one lower-cut.

Theorem 1.1 For $(m, x)$ and $(n, y) \in \mathbf{P}$, the determinantal process of red dots is given by the kernel, involving at most $r+2$-fold integrals, with $r$ as in (5).

$$
\begin{aligned}
& \mathbb{K}^{\text {red }}(m, x ; n, y) \\
& =-\frac{(y-x+1)_{n-m-1}}{(n-m-1) !} \mathbb{1}_{n>m} \mathbb{1}_{y \geq x}+\frac{(N-n) !}{(N-m-1) !} \oint_{\Gamma(x+\mathbb{N})} \frac{d v(v-x+1)_{N-m-1}}{2 \pi \mathrm{i} Q_{\mathcal{R}}(v) Q_{\mathfrak{C}}(v)} \times \\
& \left(\oint_{\Gamma_{\infty}} \frac{d z Q_{\mathcal{R}}(z) Q_{\mathfrak{C}}(z)}{2 \pi \mathrm{i}(z-v)(z-y)_{N-n+1}} \frac{\Omega_{r}^{+}(v, z)}{\Omega_{r}^{+}(0,0)}+\frac{1}{r+1} \oint_{\Gamma_{\tau}} \frac{d z P(z) Q_{\mathcal{L}}(z)}{2 \pi \mathrm{i}(z-y)_{N-n+1}} \frac{\Omega_{r+1}^{-}(v, z)}{\Omega_{r}^{+}(0,0)}\right),
\end{aligned}
$$

where

$$
\begin{aligned}
\Gamma(x+\mathbb{N}) & :=\text { contour containing the set } x+\mathbb{N}=\{x, x+1, \ldots\} \\
\Gamma_{\infty} & :=\text { very large contour containing all the poles of the } z \text {-integrand } \\
\Gamma_{\tau} & :=\Gamma\left(y+n-N, \ldots, \min \left(y_{1}-N, y\right)\right) \mathbb{1}_{\tau<0}, \text { with } \tau:=(y+n)-\left(y_{1}+1\right) .
\end{aligned}
$$

It is an interesting open problem to investigate the correlation function $\operatorname{det}\left(\mathbb{K}^{\mathrm{red}}\left(m_{i}, x_{i} ; n_{j}, y_{j}\right)\right)_{1 \leq i, j \leq k}$ in the bulk (liquid region) and its limit when the size of the figure tends uniformly to $\infty$. For a configuration with cuts on one side only, Petrov [37] has shown that the limit of the correlation function is given by the correlation of the incomplete beta-kernel for a "slope" satisfying the Burger's equation (translation invariant Gibbs measure); these were introduced in [35. Gorin [25] goes beyond by showing that the result remains valid if one allows the location of the cuts on one side to be random, yielding a measure in the limit. For a figure with two-sided cuts (above and below), what is the analogue of the incomplete beta-kernel and the slope? The present project actually deals with a very different limit, as will be explained below.

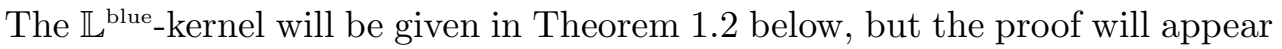
in another paper [3]. It is not clear how to obtain the $\mathbb{L}^{\text {blue }}$-kernel from scratch, due to the intricacy of the interlacing pattern, mentioned earlier. Therefore we must first compute the $\mathbb{K}^{\text {red }}$-kernel and then hope to compute the $\mathbb{L}^{\text {blue }}$-kernel by an alternative method. Indeed, we check that the inverse Kasteleyn matrix of the dimers on the associated bipartite graph dual to $\mathbf{P}$ coincides with the $\mathbb{K}$-kernel. This leads us to the following statement to appear in [3]: 
Theorem 1.2 The $\mathbb{L}^{\text {blue }}$-process of blue dots and the $\mathbb{K}^{\text {red }}$-process of red dots have kernels related as follows:

$$
\mathbb{L}^{\text {blue }}\left(\eta, \xi ; \eta^{\prime}, \xi^{\prime}\right)=-\mathbb{K}^{\text {red }}\left(m-\frac{1}{2}, x ; m^{\prime}+\frac{1}{2}, x^{\prime}\right),
$$

where $(m, x)$ and $\left(m^{\prime}, x^{\prime}\right)$ are the same geometric points as $(\eta, \xi)$ and $\left(\eta^{\prime}, \xi^{\prime}\right)$, expressed in the new coordinates (1); see Fig. 6.

We state the asymptotic result (without proof), suggested by the simulation of Fig.5, for the two-cut model (one cut below and one above) and for even $N \rightarrow \infty$. We do not expect the multi-cut case to lead to a fundamentally new universality class in the limit, as the upper- and lower-cuts will only interact pairwise locally. For the two-cut model, we concentrate on the polygonal shape $\mathbf{P}$, as in Figs.2\&4, with $\ell=1, u=2$ and with equal opposite parallel sides, i.e., $b_{u}=d_{0}$ and $N-b_{u}=b_{0}$; it has two cuts of same size $d:=d_{1}=b_{1}$, one on top and one at the bottom.

For that model, the oblique strip $\{\rho\}$ extending the oblique segments of the upper- and lower-cuts within $\mathbf{P}$ will play an important role (see Fig.4): that is the region containing the parallel lines $x+n=-\frac{1}{2}+k$ (or, what is the same, the lines $\eta=k)$ for $m_{1} \leq k \leq n_{1}+b-d$. Thus the strip $\{\rho\}$ has the following width:

$$
\rho:=n_{1}-m_{1}+b-d=m_{2}-n_{2}+b-d, \text { setting } b:=b_{0},
$$

and we assume $\rho \geq 0$. It will be shown that the $\rho+1$ parallel oblique lines $\eta=k, k \in \mathbb{Z}$ within the strip $\{\rho\}$ each carry the same number $r$ of blue dots, with $r$ defined in (5). In the simulation of Fig.5, $\{\rho\}$ is the "finite" oblique strip separating the two "large" hexagons; see also Fig.4.

The discrete-tacnode kernel $\mathbb{L}^{\mathrm{dTac}}\left(\tau_{1}, \theta_{1} ; \tau_{2}, \theta_{2}\right)$ in the variables $\left(\tau_{i}, \theta_{i}\right) \in \mathbb{Z} \times \mathbb{R}$ is defined by the following expression, where the integrations are taken along upwards oriented vertical lines $\uparrow L_{0+}$ to the right of a (counterclock) contour $\Gamma_{0}$ about the origin and with integer $r \geq 0$ :

$$
\begin{aligned}
\mathbb{L}^{\mathrm{dTac}}\left(\tau_{1}, \theta_{1} ; \tau_{2}, \theta_{2}\right):=-\mathbb{H}^{\tau_{1}-\tau_{2}}\left(\theta_{2}-\theta_{1}\right) & \\
& +\oint_{\Gamma_{0}} \frac{d V}{(2 \pi \mathrm{i})^{2}} \oint_{\uparrow L_{0+}} \frac{d Z}{Z-V} \frac{V^{\rho-\tau_{1}}}{Z^{\rho-\tau_{2}}} \frac{e^{-V^{2}-\theta_{1} V}}{e^{-Z^{2}-\theta_{2} Z}} \frac{\Theta_{r}(V, Z)}{\Theta_{r}(0,0)} \\
& +\oint_{\Gamma_{0}} \frac{d V}{(2 \pi \mathrm{i})^{2}} \oint_{\uparrow L_{0+}} \frac{d Z}{Z-V} \frac{V^{\tau_{2}}}{Z^{\tau_{1}}} \frac{e^{-V^{2}+\left(\theta_{2}-\beta\right) V}}{e^{-Z^{2}+\left(\theta_{1}-\beta\right) Z}} \frac{\Theta_{r}(V, Z)}{\Theta_{r}(0,0)} \\
& +r \oint_{\uparrow L_{0+}} \frac{d V}{(2 \pi \mathrm{i})^{2}} \oint_{\uparrow L_{0+}} d Z \frac{V^{-\tau_{1}}}{Z^{\rho-\tau_{2}}} \frac{e^{V^{2}-\left(\theta_{1}-\beta\right) V}}{e^{-Z^{2}-\theta_{2} Z}} \frac{\Theta_{r-1}^{+}(V, Z)}{\Theta_{r}(0,0)} \\
& -\frac{1}{r+1} \oint_{\Gamma_{0}} \frac{d V}{(2 \pi \mathrm{i})^{2}} \oint_{\Gamma_{0}} d Z \frac{V^{\rho-\tau_{1}}}{Z^{-\tau_{2}}} \frac{e^{-V^{2}-\theta_{1} V}}{e^{Z^{2}-\left(\theta_{2}-\beta\right) Z}} \frac{\Theta_{r+1}^{-}(V, Z)}{\Theta_{r}(0,0)}=: \sum_{0}^{4} \mathbb{L}_{i}^{\mathrm{dTac}},
\end{aligned}
$$




$\begin{array}{lll}\mathrm{h} \quad \mathrm{h}+1 & \mathrm{~h} & \mathrm{~h} \\ \mathrm{~h}{ }_{\mathrm{hlue}}^{\mathrm{h}+1} & \mathrm{~h} \cdot \mathrm{h} & \mathrm{h} \\ \mathrm{h} & \text { red } & \text { green }\end{array}$

Fig. 3. Three types of tiles, with the height function and with corresponding level line. The red tiles (blue tiles) have a red dot (blue dot) in the middle.

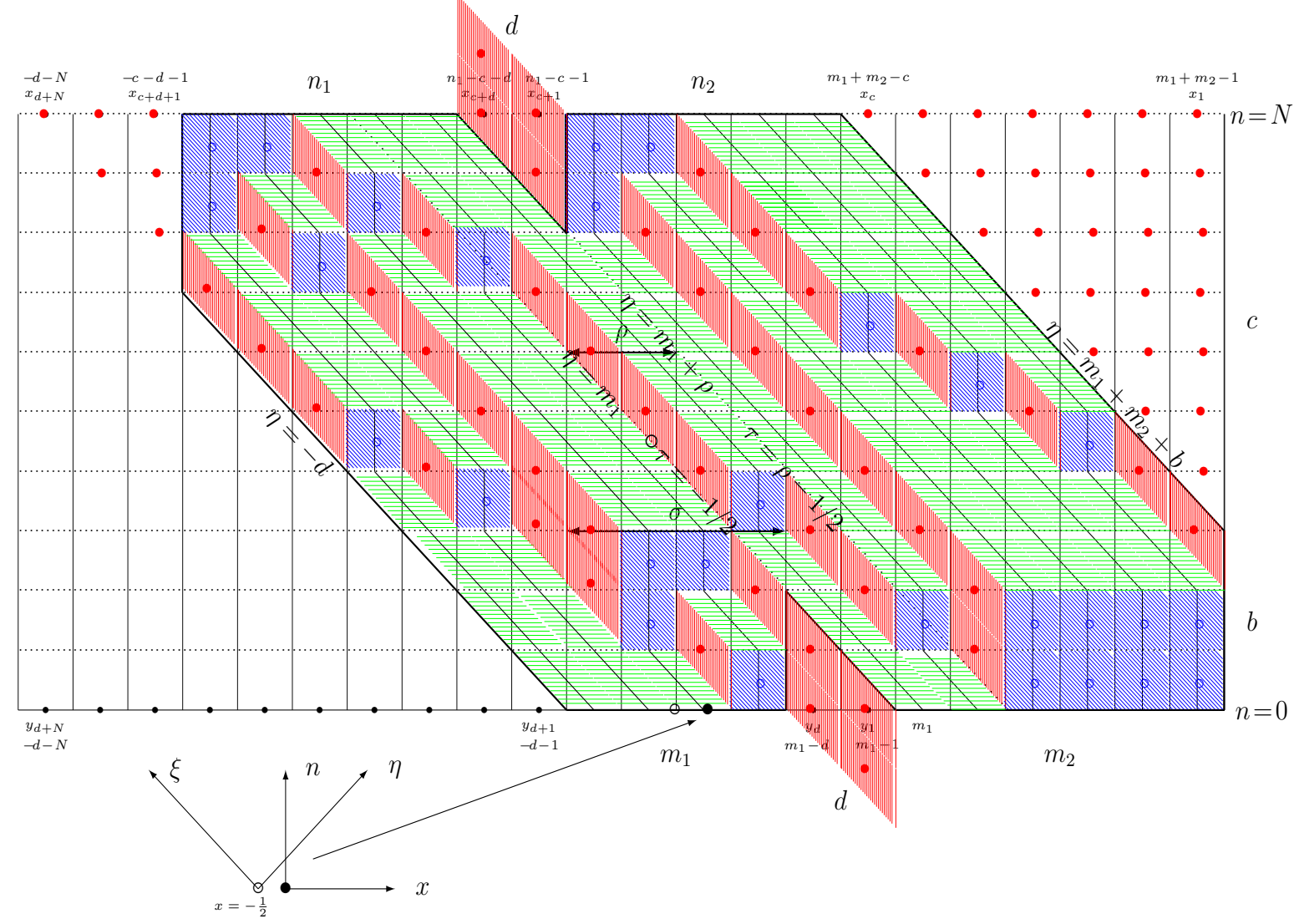

Fig. 4 : Tiling of a hexagon with two opposite cuts of equal size (Twocut model), with red, blue and green tiles. Here $d=2, n_{1}=n_{2}=5, m_{1}=$ $4, m_{2}=6, b=3, c=7$, and thus $r=1, \rho=2, \sigma=4$. The $(x, n)$-coordinates have their origin at the black dot and the $(\xi, \eta)$-coordinates at the circle given by $(x, n)=\left(-\frac{1}{2}, 0\right)$. Red tiles carry red dots on horizontal lines $n=k$ for $0 \leq k \leq N$ ( $\mathbb{K}$-process) and blue tiles blue dots on oblique lines $\eta=k$ for $-d+1 \leq k \leq m_{1}+m_{2}+b-1$ (L्L-process). The left and right boundaries of the strip $\{\rho\}$ are given by the dotted oblique lines $\eta=m_{1}$ and $\eta=m_{1}+\rho$. Finally, the tilings define $m_{1}+m_{2}$ non-intersecting paths. 

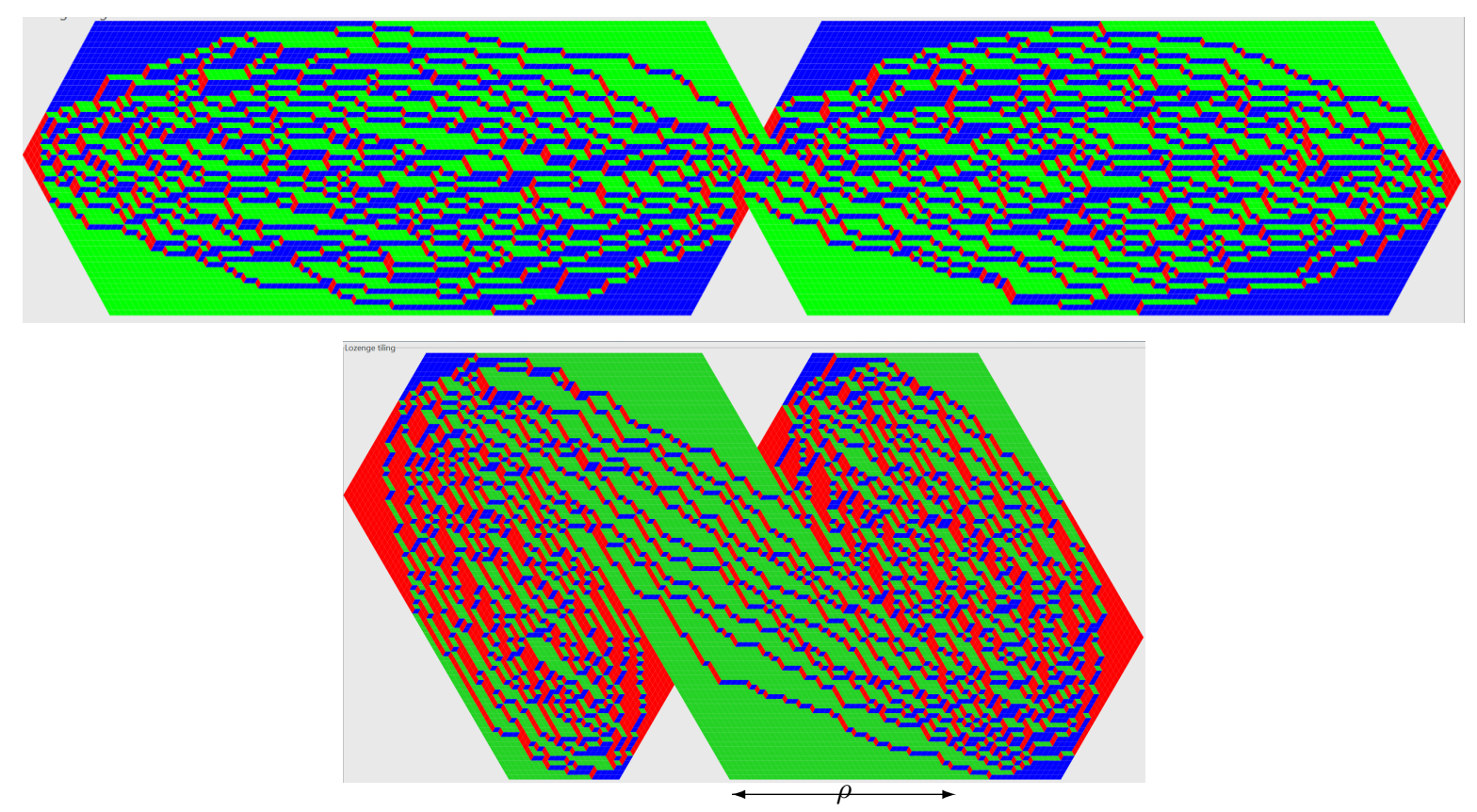

Fig. 5. Computer simulation for $n_{1}=105, n_{2}=95, m_{1}=m_{2}=100, b=25, c=$ $30, d=20$ and $n_{1}=50, n_{2}=30, m_{1}=20, m_{2}=60, b=30, c=60, d=20$. Courtesy of Antoine Doeraene.
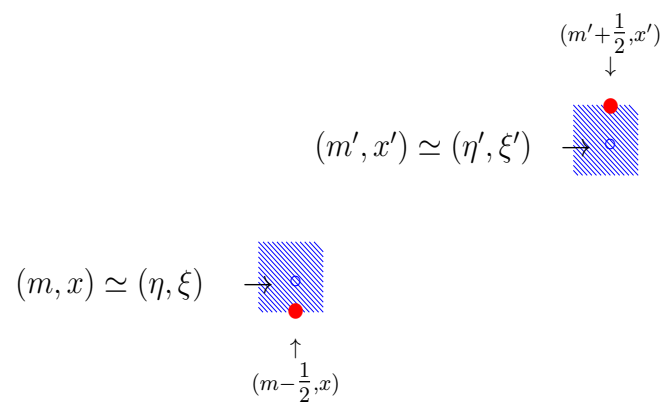

Fig. 6. The $\mathbb{L}^{\text {blue }}$-kernel of blue dots expressed in terms of the $\mathbb{K}^{\text {red-kernel of }}$ neighbouring red dots, using the $(\eta, \xi)$-variables in (1).

where

$$
\begin{aligned}
& \mathbb{H}^{m}(z):=\frac{z^{m-1}}{(m-1) !} \mathbb{1}_{z \geq 0} \mathbb{1}_{m \geq 1}, \quad \text { (Heaviside function) } \\
& \Theta_{r}(V, Z):=\left[\prod_{1}^{r} \oint_{\uparrow L_{0+}} \frac{e^{2 W_{\alpha}^{2}+\beta W_{\alpha}}}{W_{\alpha}^{\rho}}\left(\frac{Z-W_{\alpha}}{V-W_{\alpha}}\right) \frac{d W_{\alpha}}{2 \pi \mathrm{i}}\right] \Delta_{r}^{2}\left(W_{1}, \ldots, W_{r}\right) \\
& \Theta_{r \mp 1}^{ \pm}(V, Z):=\left[\prod_{1}^{r \mp 1} \oint_{\uparrow L_{0+}} \frac{e^{2 W_{\alpha}^{2}+\beta W_{\alpha}}}{W_{\alpha}^{\rho}}\left(\left(Z-W_{\alpha}\right)\left(V-W_{\alpha}\right)\right)^{ \pm 1} \frac{d W_{\alpha}}{2 \pi \mathrm{i}}\right] \\
& \Delta_{r \mp 1}^{2}\left(W_{1}, \ldots, W_{r \mp 1}\right) .
\end{aligned}
$$

We now state the main Theorem of this project, which will appear in [3]: 
Theorem 1.3 Given the polygon $\mathbf{P}$ with cuts of equal size d, above and below, keeping $\rho, r \geq 0$ fixed, as defined in (12),(5), let the polygon $\mathbf{P}$ and the size $d$ of the two cuts go to $\infty$, according to the following scaling of the geometric variables $b, c, m_{i}, n_{i}>0$, in terms of $d \rightarrow \infty$ and new parameters $1<\gamma<3, a:=2 \sqrt{\frac{\gamma}{\gamma-1}}$, $\beta_{1}<0, \beta_{2}, \gamma_{1}, \gamma_{2} \in \mathbb{R}$,

$$
\begin{array}{ll}
b=d+r & c=\gamma d \\
n_{i}=m_{i}-(-1)^{i}(\rho-r) & m_{i}=\frac{\gamma+1}{\gamma-1}\left(d+\frac{a}{2} \beta_{i} \sqrt{d}+\gamma_{i}\right) \text { for } i=1,2 .
\end{array}
$$

The variables $(\eta, \xi) \in \mathbb{Z}^{2}$ with $\xi-\eta \in 2 \mathbb{Z}+1$ get rescaled into new variables $(\tau, \theta) \in \mathbb{Z} \times \mathbb{R}$, having their origin at the halfway point $\left(\eta_{0}, \xi_{0}\right)$ along the left boundary of the strip $\{\rho\}$, shifted by $\left(-\frac{1}{2}, \frac{1}{2}\right)$ (see little circle along the line $\eta=m_{1}$ in Fig.4):

$$
\left(\eta_{i}, \xi_{i}\right)=\left(\eta_{0}, \xi_{0}\right)+\left(\tau_{i}, \frac{\gamma+1}{a}\left(\theta_{i}+\beta_{2}\right) \sqrt{d}\right) \text { with }\left(\eta_{0}, \xi_{0}\right)=\left(m_{1}, N-m_{1}-1\right) .
$$

With this scaling and after a conjugation, the kernel (11) of the $\mathbb{L}$-process tends to the new kernel $\mathbb{L}^{d T a c}$, as in (13), depending only on the width $\rho$ of the strip $\{\rho\}$, the number $r=b-d$ of blue dots on the oblique lines in the strip $\{\rho\}$ and the parameter $\beta:=-\beta_{1}-\beta_{2}$, to be precise,

$$
\begin{aligned}
\lim _{d \rightarrow \infty}(-1)^{\frac{1}{2}\left(\eta_{1}+\xi_{1}-\eta_{2}-\xi_{2}\right)} & \left(\sqrt{d} \frac{\gamma+1}{2 a}\right)^{\eta_{2}-\eta_{1}} \mathbb{L}^{\text {blue }}\left(\eta_{1}, \xi_{1} ; \eta_{2}, \xi_{2}\right) \frac{1}{2} \Delta \xi_{2} \\
& =\mathbb{L}^{d T a c}\left(\tau_{1}, \theta_{1} ; \tau_{2}, \theta_{2}\right) d \theta_{2} .
\end{aligned}
$$

The kernel satisfies the following involution:

$$
\mathbb{L}^{d T a c}\left(\tau_{1}, \theta_{1} ; \tau_{2}, \theta_{2}\right)=\mathbb{L}^{d T a c}\left(\rho-\tau_{2}, \beta-\theta_{2} ; \rho-\tau_{1}, \beta-\theta_{1}\right) .
$$

This involution exchanges $\mathbb{L}_{1}^{d T a c} \leftrightarrow \mathbb{L}_{2}^{d T a c}$, with $\mathbb{L}_{k}^{d T a c}$ being self-involutive for $k=$ 3, 4. Also $\mathbb{L}_{1}^{d T a c}$ has support on $\left\{\tau_{1}>\rho\right\}, \mathbb{L}_{2}^{d T a c}$ has support on $\left\{\tau_{2}<0\right\}$ and $\mathbb{L}_{4}^{\text {dTac }}$ on $\left\{\tau_{1}>\rho\right\} \cap\left\{\tau_{2}<0\right\}$.

These formulas of Theorems 1.1 and 1.2 can be specialized to known situations: to hexagons with no cuts (Johansson[20]), to non-convex polygons $\mathbf{P}$ with cuts at the top only (Petrov [38]), and to the case where the strip $\rho$ reduces to a line (i.e., $\rho=0$ ), in which the polygon $\mathbf{P}$ (as in Fig.3) can be viewed as two hexagons glued together along one side (Duse-Metcalfe [15]).

Outline. Many steps are necessary to prove Theorem 1.1 .

Section 2: Instead of putting the uniform distribution on the red dot-configuration on $\mathbf{P}$, it will be more convenient to first consider a non-uniform distribution depending on a parameter $0<q \leq 1$; this distribution will tend to the uniform 
one when $q \rightarrow 1$. The red dot-configuration will be shown to be equivalent to the set of semi-standard skew-Young Tableaux of a given shape; the latter can be read off the geometry of $\mathbf{P}$. The $q$-probability on this set will have a KarlinMcGregor type of formula, which is not surprising due an equivalent formulation in terms of non-intersecting paths. The use of $q$-deformations have been initiated by Okounkov-Reshetikhin [36] and Kenyon-Okounkov [28]. Also it has been used effectively in the work of Petrov [38] for lozenge tilings of hexagons with cuts on the upper-side only. Section 2 also contains a brief description of the two-cut model.

Section 3 deals with some determinantal identities, but also with a useful, but unusual, integral representation of the elementary symmetric function $h_{y-y_{j}}\left(1^{n}\right)$. Section 4: Adapting Eynard-Mehta techniques, further refined in [9] and [7], will lead to the construction a kernel $\mathbb{K}_{q}$, involving the inverse of a matrix $M$.

Section 5: The matrix $M$ can be transformed so that the inverse is readily computable.

Section 6: The transformed kernel $\mathbb{K}_{q}$ has a multiple integral representation, using integral representations of the different ingredients.

Section 7: Taking the limit when $q \rightarrow 1$ yields a kernel $\mathbb{K}^{\text {red }}$, involving contour integrals about $\mathcal{R}$, with at the worst $d+2$-fold integrations, where $d$ is the size of the cuts.

Section 8: The kernel $\mathbb{K}^{\text {red }}$ will then further be reduced to a sum of contour integrations, mostly about $\mathcal{L}$, with at the worst $r+3$-fold integrals.

\section{Interlacing and measures on skew-Young Tableaux}

The two-cut model, already mentioned before, deserves some further discussion. Remember for the two-cut model we have $b:=b_{0}=|\mathcal{L}|$ and $c:=b_{u}$; see Fig. 2\&4. In other terms, this is now a hexagon with edges of size $m_{1}+m_{2}+d, b, c \sqrt{2}, n_{1}+n_{2}+d, b, c \sqrt{2}$ with two cuts, one below and one above, both of same size $d$, satisfying $m_{1}+m_{2}=n_{1}+n_{2}$ and with $N=b+c$ (see Fig.4). The quadrilateral $\widetilde{\mathbf{P}}$ associated with $\mathbf{P}$ is depicted in Fig.7. Note that the most-right point $y_{1}=m_{1}-1$ in the lower-cut plays an important role!

In the two-cut model, two strips within $\mathbf{P}$ will play a special role: (Fig.4) (i) the oblique strip $\{\rho\}$, already introduced in $(12)$.

(ii) a vertical strip $\{\sigma\}$ extending the vertical segments of the upper- and lowercuts; that is the region between the lines $x=n_{1}-c-\frac{1}{2}$ and $x=m_{1}-d-\frac{1}{2}$. The strip $\{\sigma\}$ has width (again same notation for the name and the width of the strip!)

$$
\sigma:=m_{1}-n_{1}+c-d=n_{2}-m_{2}+c-d \geq 0 .
$$




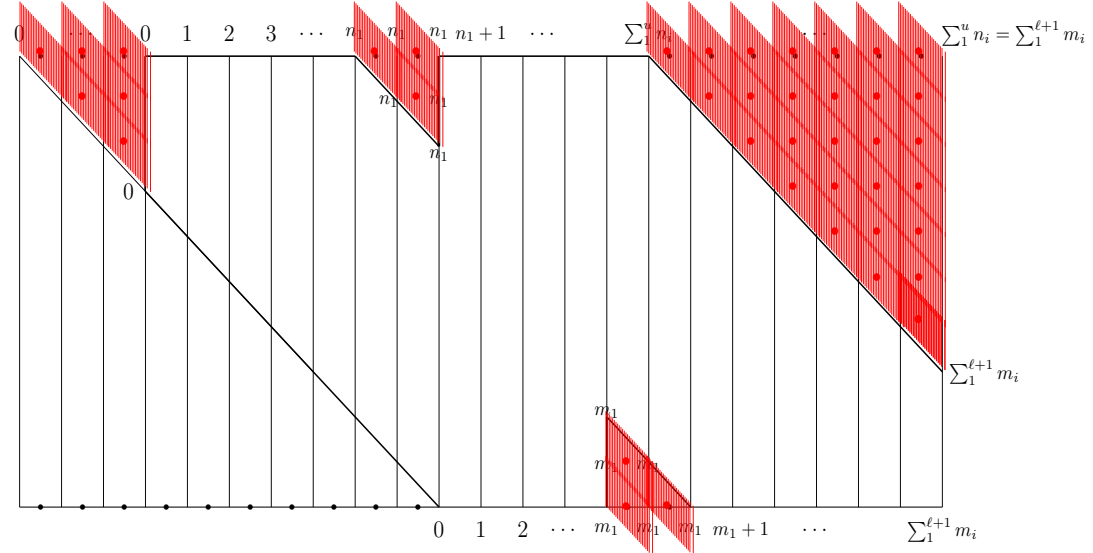

Fig. 7. The polygon $\mathbf{P}$, with $u-1$ upper-cuts and $\ell$ lower-cuts and the parallelogram $\widetilde{\mathbf{P}}=\mathbf{P} \cup\{4$ red triangles $\}$. The heights along the boundary of $\mathbf{P}$ are given by the numbers next to the figure.

That $\rho, \sigma \geq 0$ amounts to the inequalities:

$$
d-b \leq n_{1}-m_{1}=m_{2}-n_{2} \leq c-d .
$$

It is natural to assume that the strips $\{\rho\}$ (respectively $\{\sigma\}$ ) have no point in common with the vertical parts (respectively oblique parts) of the boundary $\partial \mathbf{P}$. This condition for $\{\rho\}$ implies $\left\{x+n=-\frac{1}{2}+m_{1}\right\} \cap\{n=N\}>-c-d-\frac{1}{2}$ and $\left\{x+n=-\frac{1}{2}+n_{1}+b-d\right\} \cap\{n=0\}<m_{1}+m_{2}-\frac{1}{2}$, implying $d-b>$ $\max \left(-m_{1},-n_{2}\right)$. The condition for the strip $\{\sigma\}$ implies $n_{1}-c-\frac{1}{2}>-d-\frac{1}{2}$ and $m_{1}-d-\frac{1}{2}<m_{1}+m_{2}-c-\frac{1}{2}$, implying $c-d<\min \left(n_{1}, m_{2}\right)$. These inequalities combined with condition $\rho, \sigma \geq 0$ above imply

$$
\max \left(-n_{2},-m_{1}\right)<d-b \leq m_{2}-n_{2}=n_{1}-m_{1} \leq c-d<\min \left(m_{2}, n_{1}\right) .
$$

The four regions $\subset$ line $\{n=N\}$ mentioned in (3) can now be written as

$$
\begin{aligned}
\mathcal{L} & =\left\{x_{d+c+b}, \ldots, x_{d+c+1}\right\}, \quad \mathcal{R}=\left\{x_{c}, \ldots, x_{1}\right\}, \quad \mathcal{C}=\left\{x_{c+d}, \ldots, x_{c+1}\right\} \\
\mathcal{G} & =\{\rho\} \cup\{\sigma\},
\end{aligned}
$$

and so the polynomials $P$ and $Q$, as in (4), spelled out, are given by:

$$
\begin{aligned}
& P(z)=: P_{\rho}(z) Q_{\mathfrak{C}}(z) P_{\sigma}(z)=\left(z-x_{d+c}+1\right)_{\rho}\left(z-x_{c+1}\right)_{d}\left(z-y_{d}+1\right)_{\sigma} \\
& Q(z):=Q_{\mathcal{L}}(z) Q_{\mathcal{C}}(z) Q_{\mathcal{R}}(z)=\left(z-x_{d+c+1}\right)_{b}\left(z-x_{c+1}\right)_{d}\left(z-x_{1}\right)_{c},
\end{aligned}
$$

where $P_{\rho}, P_{\sigma}, Q_{\mathcal{L}}, Q_{\mathcal{C}}, Q_{\mathcal{R}}$ are monic polynomials whose roots are given by the sets $\{\bar{\rho}\},\{\bar{\sigma}\}, \mathcal{L}, \mathcal{C}$ and $\mathcal{R}$, respectively, where $\{\bar{\rho}\}:=\{\rho\} \cap\{n=N\}$ and $\{\bar{\sigma}\}:=\{\sigma\} \cap\{n=N\}$.

The inequalities $(19)$ imply that each of the sets $\mathcal{C} \cup\{\bar{\sigma}\}$ and $\{\bar{\rho}\} \cup \mathcal{C}$ form a contiguous set of integers, such that each of the three sets are completely separated: $\mathcal{C}<\{\bar{\sigma}\}<\mathcal{R}$ and $\mathcal{L}<\{\bar{\rho}\}<\mathcal{C}$. 
Nonintersecting paths, level lines and the $\mathbb{K}^{\text {red }}$ - and $\mathbb{L}^{\text {blue }}$-processes. The height function on the tiles, given in Fig.3, imply that the heights along the boundary of the polygon $\mathbf{P}$ are independent of the tiling; for the heights along $\partial \mathbf{P}$, see Fig. 7. The level lines of heights $\frac{1}{2}, \frac{3}{2}, \ldots, m_{1}-\frac{1}{2}, m_{1}+\frac{1}{2}, \ldots, \sum_{1}^{\ell+1} m_{i}-\frac{1}{2}$ pass obliquely through green tiles, vertically through blue tiles, and avoid the red tiles; the level lines are the nonintersecting paths going from top to bottom in Fig.4. It follows that the intersection (within $\mathbf{P}$ ) of the level lines with the oblique lines $\{\eta=$ integer $\}$ determine the blue dots and with the lines $\{n=$ integer $\}$ the red dot at the integers $x \notin$ level lines. In other terms drawing a horizontal line from left to right through the middle of the blue and green tiles (say, in Fig. 4) increases the height by 1 and remains flat along the red tiles. Thus the tilings of the hexagon $\mathbf{P}$ are equivalent to $\sum_{1}^{\ell+1} m_{i}=\sum_{1}^{u} n_{i}$ non-intersecting level-lines. It follows that

$$
\begin{aligned}
& \#\{\text { red dots of the } \mathbb{K} \text {-process on the horizontal line } n=k \text { within } \widetilde{\mathbf{P}}\} \\
& =\#\{\text { flat segments of the heigth function along the line } n=k\} \\
& =[\text { length of the }(\text { line } n=k) \cap \widetilde{\mathbf{P}}]-\left[\begin{array}{l}
\text { increment in height between the } \\
\text { two points }(\text { line } n=k) \cap \partial \widetilde{\mathbf{P}}
\end{array}\right] \\
& =\left(\sum_{1}^{\ell+1} m_{i}+d+k\right)-\left(\sum_{1}^{\ell+1} m_{i}\right)=d+k .
\end{aligned}
$$

When an oblique line $\eta=k$ traverses a tile, as in Fig.2, the height increases by 1 for a blue tile and stays flat for a red and green tile. Therefore, we have

$$
\begin{aligned}
& \#\{\text { blue dots of the } \mathbb{L} \text {-process on the oblique line } \eta=k \text { within } \widetilde{\mathbf{P}}\} \\
& \quad=[\text { increment in height along }(\text { line } \eta=k) \cap \partial \widetilde{\mathbf{P}}]
\end{aligned}
$$


leading to the following pattern for the blue dots for the two-cut model:

$\begin{array}{cc}k & \#\{\text { blue dots on } \eta \\ n_{1}+n_{2}+b & 0 \\ \vdots & \\ n_{1}+n_{2} & b \\ \vdots & \\ n_{1}+b & b \\ \vdots & \\ m_{1}+\rho & b-d=r \\ \vdots & \\ m_{1} & b-d=r \\ \vdots & \\ m_{1}-d & b \\ \vdots & \\ b-d & b \\ \vdots & \\ -d & 0\end{array}$

This implies that the number of blue dots are $=r$ (local minimum) along each of the oblique lines $\eta=k$ within the strip $\{\rho\}$, including its boundary. Outside, that number starts growing linearly in steps of 1 up to $b$, stays fixed for a while and then goes down to 0 linearly in steps of 1 .

The red dot-configurations and skew-Young tableaux. The red dots on the horizontal lines $\{n=k\} \cap \widetilde{\mathbf{P}}$ for $0 \leq k \leq N$ are parametrized by $x^{(k)} \in \mathbb{Z}^{d+k}$

$$
x_{N}^{(k)}<\ldots<x_{1}^{(k)},
$$

subjected to the interlacing pattern below, in short $x^{(k-1)} \prec x^{(k)}$,

$$
x_{i+1}^{(k)}<x_{i}^{(k-1)} \leq x_{i}^{(k)} .
$$

This interlacing follows from an argument similar to [38, 1]. So, we have a truncated Gelfand-Tsetlin cone with prescribed top and bottom and for completion, set $x_{k}^{(0)}=y_{k}$ and $x_{k}^{(N)}=x_{k}$ :

$$
\begin{aligned}
& x_{d+N}<\ldots<x_{d+c}<\ldots<x_{1+c}<\ldots<x_{1} \\
& x_{d+N-1}^{(N-1)}<\quad \ldots \quad<x_{1}^{(N-1)} \\
& y_{d}<\cdots<y_{2}<\cdots<y_{1} \text {. }
\end{aligned}
$$


The interlacing pattern above, with $x^{(k-1)} \prec x^{(k)}$, is equivalent to a red dot configuration of a polygon $\mathbf{P}$.

As an example, for the two-cut model, the top consists of the three regions $\mathcal{L}, \mathcal{C}$ and $\mathcal{R}$ of contiguous integers; and the bottom of one contiguous region, given by the lower-cut.

Moreover, setting for $0 \leq k \leq N, 4$

$$
\nu_{i}^{(k)}=x_{i}^{(k)}+i
$$

leads to a sequence of partitions

$$
\nu^{(0)} \subset \nu^{(1)} \ldots \subset \nu^{(N)},
$$

with prescribed initial and final condition,

$$
\mu:=\nu^{(0)} \quad \text { and } \quad \lambda:=\nu^{(N)}
$$

and such that each skew diagram $\nu^{(i)} \backslash \nu^{(i-1)}$ is a horizontal strip ${ }^{5}$. Note the condition $x_{i} \geq y_{i}$ for all $1 \leq i \leq d+N$ on the cuts, mentioned just before (2), guarantees that $\mu \subset \lambda$. In fact the consecutive diagrams $\nu^{(i)} \backslash \nu^{(i-1)}$ are horizontal strips, if and only if the precise inequalities $x_{i+1}^{(m)}<x_{i}^{(m-1)} \leq x_{i}^{(m)}$ hold.

Putting the integer $i$ 's in each skew-diagram $\nu^{(i)} \backslash \nu^{(i-1)}$, for $1 \leq i \leq N$, is equivalent to a skew-Young tableau filled with $N$ numbers $1, \ldots, N$ and exactly $N$. This leads to the following semi-standard skew-Young tableau in the two-cut case, as in Fig.8. An argument, using the height function, which is similar to the one in [1, shows that all configurations are equally likely. So we have uniform distribution on the set of configurations. To conclude, we have:

\{All configuration of red dots\}

$\Longleftrightarrow$ \{skew-Young Tableaux of shape $\lambda \backslash \mu$ filled with numbers 1 to $N$ \}

$$
\Longleftrightarrow\left\{\begin{array}{l}
\mu=\nu^{(0)} \subset \nu^{(1)} \ldots \subset \nu^{(N)}=\lambda, \text { with } \mu \subset \lambda \text { fixed, } \\
\text { with } \nu^{(m)} \backslash \nu^{(m-1)}=\text { horizontal strip, }
\end{array}\right\}
$$

${ }^{4}$ Define $\left|x^{(k)}\right|:=\sum_{i=1}^{d+k} x_{i}^{(k)}$ for $0 \leq k \leq N$; in particular for $k=0$ and $k=N$, we have $|y|=\sum_{1}^{d} y_{i}$ and $|x|=\sum_{1}^{d+N} x_{i}$. That means, the sum is always taken within the parallelogram $\widetilde{\mathbf{P}}$. Also define $\left|\nu^{(k)}\right|:=\sum_{i} \nu_{i}^{(k)}$

${ }^{5}$ An horizontal strip is a Young diagram where each row has at most one box in each column. 
with equal probability for each configuration.

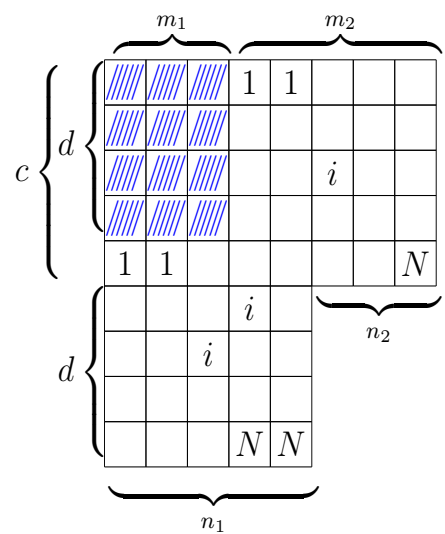

\begin{tabular}{|c|c|c|c|c|c|c|c|c|}
\hline & \multirow{2}{*}{\begin{tabular}{l|l}
1 & 1 \\
2 & 3 \\
\end{tabular}} & \multirow{2}{*}{\begin{tabular}{|l|}
3 \\
4 \\
\end{tabular}} & & \multirow{2}{*}{\multicolumn{2}{|c|}{\begin{tabular}{|l|l|}
3 & 3 \\
4 & 4 \\
\end{tabular}}} \\
\hline & & & & & \\
\hline \multicolumn{4}{|c|}{ 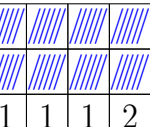 } & 32 & 45 & 5 & 5 & \\
\hline & & & 5 & & & & \\
\hline \multicolumn{2}{|r|}{\begin{tabular}{|l|}
2 \\
3 \\
\end{tabular}} & & & 6 & & 8 & 8 & \\
\hline & \begin{tabular}{|l|}
3 \\
5 \\
\end{tabular} & 6 & 6 & 7 & 8 & 9 & 99 & \\
\hline \multicolumn{2}{|c|}{\begin{tabular}{|l|l}
5 \\
\end{tabular}} & 7 & 7 & 8 & 9 & & & \\
\hline \multicolumn{2}{|c|}{77} & 8 & 9 & & & & & \\
\hline 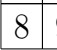 & & & & \\
\hline
\end{tabular}

Fig. 8: Example for the two-cut model. Semi-standard skew-Young Tableau $\lambda \backslash \mu$ filled with numbers $1, \cdots, N$ in one-to one correspondence with the red dotprocess. On the right is the precise semi-standard skew-Young Tableau associated with Fig. 4.

Uniform and $q$-probability on the set of red dot-configurations. The last statement above implies that

$$
\#\{\text { configuration of red dots }\}=s_{\lambda \backslash \mu}(\underbrace{1, \ldots, 1}_{N}, 0,0 \ldots),
$$

and thus we have uniform probability measure $\mathbb{P}$ on the space of all $N+1$ uples of partitions $\left\{\nu^{(0)}, \ldots, \nu^{(N)}\right\}$

$\mathbb{P}$ (given configuration of red dots)

$$
\begin{aligned}
& =\mathbb{P}\left(\nu^{(0)}, \nu^{(2)}, \ldots \nu^{(N)}, \text { such that } \mu=\nu^{(0)} \subset \nu^{(1)} \subset \ldots \subset \nu^{(N)}=\lambda\right) \\
& =\frac{1}{s_{\lambda \backslash \mu}(\underbrace{1, \ldots, 1}_{N}, 0,0 \ldots)} \mathbb{1}_{\nu^{(0)} \subset \nu^{(1)} \subset \ldots \subset \nu^{(N)}} \mathbb{1}_{\nu^{(0)}=\mu} \mathbb{1}_{\nu^{(N)}=\lambda} .
\end{aligned}
$$

As will be seen, we define a $q$-dependent probability measure $\mathbb{P}_{q}$, for $0<q \leq 1$, on the same space of all $N+1$-uples of partitions, as follows

$$
\begin{aligned}
& \mathbb{P}_{q}\left(\nu^{(0)}, \nu^{(2)}, \ldots \nu^{(N)}, \text { such that } \mu=\nu^{(0)} \subset \nu^{(1)} \subset \ldots \subset \nu^{(N)}=\lambda\right) \\
& \quad=\frac{\left(q^{-1}\right)^{\sum_{i=1}^{N-1}\left|\nu^{(i)}\right|-(N-1)\left|\nu^{(0)}\right|}}{s_{\lambda \backslash \mu}\left(q^{1-N}, q^{2-N}, \ldots, q^{-1}, q^{0}\right)} \mathbb{1}_{\nu^{(0)} \subset \nu^{(1)} \subset \ldots \subset \nu^{(N)}} \mathbb{1}_{\nu^{(0)}=\mu^{1}} \mathbb{1}_{\nu^{(N)}=\lambda},
\end{aligned}
$$

which for $q \rightarrow 1$ leads to the uniform probability (25). This formula will be explained below. 
MacDonald [31] is an excellent reference for the following discussion. Recall skew-Schur polynomials are defined as: for any $\mu \subset \lambda$,

$$
s_{\lambda \backslash \mu}(x):=\operatorname{det}\left(h_{\lambda_{i}-i-\mu_{j}+j}(x)\right)_{1 \leq i, j \leq n}=\operatorname{det}\left(h_{x_{i}-y_{j}}(x)\right)_{1 \leq i, j \leq n}, \quad \text { for } n \geq \ell(\lambda),
$$

where $h_{r}(x)$ is defined for $r \geq 0$ as

$$
\prod_{i \geq 1}\left(1-x_{i} z\right)^{-1}=\sum_{r \geq 0} h_{r}(x) z^{r}
$$

and $h_{r}(x)=0$ for $r<0$. Skew Schur polyomials also satisfy the properties

$$
s_{\lambda \backslash \mu}(x, y)=\sum_{\nu} s_{\lambda \backslash \nu}(x) s_{\nu \backslash \mu}(y)
$$

and for $x=\left(x_{1}, \ldots, x_{N}\right)$,

$$
s_{\lambda \backslash \mu}\left(x_{1}, \ldots, x_{N}\right)=\sum_{\mu=\nu^{(0)} \subset \ldots \subset \nu^{(N)}=\lambda} \prod_{i=0}^{N-1} x_{i+1}^{\left|\nu^{(i+1)}\right|-\left|\nu^{(i)}\right|} .
$$

In particular, for $0<q \leq 1$, from 30

$$
\begin{aligned}
s_{\lambda \backslash \mu}\left(q^{1-N}, q^{2-N}, \ldots, q^{-1}, q^{0}\right) & =\sum_{\mu=\nu^{(0)} \subset \nu^{(1)} \subset \ldots \subset \nu^{(N)}=\lambda}\left(q^{-1}\right)^{\sum_{i=1}^{N}(N-i)\left(\left|\nu^{(i)}\right|-\left|\nu^{(i-1)}\right|\right)} \\
& =\sum_{\mu=\nu^{(0)} \subset \nu^{(1)} \subset \ldots \subset \nu^{(N)}=\lambda}\left(q^{-1}\right)^{\sum_{i=1}^{N-1}\left|\nu^{(i)}\right|-(N-1)\left|\nu^{(0)}\right|} .
\end{aligned}
$$

The expression $\sum_{i=1}^{N}(N-i)\left(\left|\nu^{(i)}\right|-\left|\nu^{(i-1)}\right|\right)$ in formula 31 is the volume of the three-dimensional figure obtained by putting $N-i$ cubes of size $1 \times 1 \times 1$ on top of the squares containing $i$ in the skew-Young diagram, for $1 \leq i \leq N-1$. This shows why (26) defines a probability measure.

Some useful formulas: Notice that

$$
h_{r}\left(\alpha^{\gamma}, \ldots, \alpha^{\gamma-N+1}\right)=\alpha^{r(\gamma-N+1)} h_{r}\left(\alpha^{0}, \ldots, \alpha^{N-1}\right)
$$

and thus for $\alpha=q^{-1}$ and $\gamma=-d$, we have

$$
h_{r}\left(q^{0}, q^{-1}, \ldots, q^{1-N}\right)=q^{r(1-N-d)} h_{r}\left(q^{d}, \ldots, q^{d+N-1}\right) .
$$

Defining for $y, z \in \mathbb{R}$ (in terms of the $q$-Pochhammer symbol $(a ; q)_{k}=\prod_{j=0}^{k-1}(1-$ $\left.a q^{j}\right)$ for $k>0$ and $=1$ for $k=0$ ),

$$
\mathcal{P}_{n}(z):=\prod_{i=1}^{n} \frac{1-z q^{i}}{1-q^{i}}=: \frac{(z q ; q)_{n}}{(q ; q)_{n}}=: \sum_{i=0}^{n} e_{i} z^{i} \text { and } \tilde{\mathcal{P}}_{n}^{y}(z):=q^{-d y} \mathcal{P}_{n}\left(z q^{-y}\right)
$$


one checks, using the generating series $(28)$ and the definition of $\mathcal{P}_{n}$, that for $n \geq 0$,

$$
\begin{aligned}
h_{r}\left(q^{0}, \ldots, q^{n}\right) & =\frac{1}{2 \pi \mathrm{i}} \oint_{\Gamma_{0}} \frac{d u}{u^{r+1}} \prod_{\ell=0}^{n} \frac{1}{1-u q^{\ell}} \\
& =\mathcal{P}_{n}\left(q^{r}\right) \text { for } r \geq 0 .
\end{aligned}
$$

This is valid for general $r \in \mathbb{Z}$ and $n, d \geq 0$, upon inserting an indicator function:

$$
\begin{aligned}
h_{r}\left(q^{d}, \ldots, q^{d+n}\right)=q^{r d} h_{r}\left(q^{0}, \ldots, q^{n}\right) & =\frac{1}{2 \pi \mathrm{i}} \oint_{\Gamma_{0}} \frac{d u}{u^{r+1}} \prod_{\ell=d}^{d+n} \frac{1}{1-u q^{\ell}} \\
& =q^{r d} \mathcal{P}_{n}\left(q^{r}\right)\left(\mathbb{1}_{r \geq-n} \text { or } \mathbb{1}_{r \geq 0}\right) .
\end{aligned}
$$

Indeed the left hand side equals 0 for $r<0$, whereas $\mathcal{P}_{n}\left(q^{r}\right)=0$ for $-n \leq r<0$, but $\neq 0$ for $r<-n$. The integral equals 0 for $r<0$. So, the identity is valid for each of the two indicator functions; both of them will be used. Also by (28),

$$
\begin{aligned}
s_{\lambda \backslash \mu}\left(q^{0}, q^{-1}, \ldots, q^{1-N}\right) & =\operatorname{det}\left(h_{x_{i}-y_{j}}\left(q^{0}, q^{-1}, \ldots, q^{1-N}\right)\right)_{i \leq i, j \leq N+d} \\
& =q^{(d+N-1)\left(\sum_{1}^{d+N} y_{i}-\sum_{1}^{d+N} x_{i}\right)} \operatorname{det}\left(h_{x_{i}-y_{j}}\left(q^{d}, \ldots, q^{d+N-1}\right)\right)_{1 \leq i, j \leq N+d} \\
& =q^{-N(d+N-1)(d+(N+1) / 2)} q^{(d+N-1)(|y|-|x|)} \operatorname{det}(H),
\end{aligned}
$$

where $H$ is a square matrix of size $d+N$ :

$$
\begin{aligned}
H=\left(H_{i j}\right)_{1 \leq i, j \leq N+d} & :=\left(h_{x_{i}-y_{j}}\left(q^{d}, q^{d+1}, \ldots, q^{d+N-1}\right)\right)_{1 \leq i, j \leq N+d} \\
& =\left(q^{\left(x_{i}-y_{j}\right) d} h_{x_{i}-y_{j}}\left(q^{0}, \ldots, q^{N-1}\right)\right)_{1 \leq i, j \leq N+d},
\end{aligned}
$$

where formula $(33)$ is used in the second line.

q-Calculus. The polynomial $Q(z)$ has a natural $q$-extension, namely (we often write $\left.q_{i}:=q^{x_{i}}\right)$

$$
Q_{q}(z):=\prod_{1}^{d+N}\left(z-q^{x_{i}}\right)=\prod_{1}^{d+N}\left(z-q_{i}\right)
$$

and from (34) we have

$$
\mathcal{P}_{N-m}\left(v q^{-x}\right)=\left.\prod_{i=1}^{N-m} \frac{1-z q^{i}}{1-q^{i}}\right|_{z=v q^{-x}}=\prod_{i=1}^{N-m} \frac{1-v q^{i-x}}{1-q^{i}}=\frac{\left(v q^{-x+1} ; q\right)_{N-m}}{(q ; q)_{N-m}} .
$$


Following Petrov [38, we define the $q$-hypergeometric function ${ }_{2} \phi_{1}$ by means of

$$
\begin{aligned}
z \sum_{k=1}^{n} z^{-k} q^{(k-1) y} \prod_{r=n+1}^{N}\left(1-q^{r-k}\right) & =:\left(q^{N-1} ; q^{-1}\right)_{N-n 2} \phi_{1}\left(q^{-1}, q^{n-1} ; q^{N-1} \mid q^{-1}, \frac{q^{y}}{z}\right) \\
& =: \Phi_{q}\left(\frac{q^{y}}{z}\right) .
\end{aligned}
$$

$\Delta_{k}$ stands for the Vandermonde determinant of size $k$.

Lemma 2.1 For any $a \in \mathbb{Z}$, remembering the definitions (38) and (34) of $Q(z)$ and $\tilde{\mathcal{P}}_{n}^{\beta}(z)$, the following limits hold

$$
\begin{gathered}
\lim _{q \rightarrow 1} \mathcal{P}_{n}\left(q^{x}\right)=\prod_{k=1}^{n} \frac{k+x}{k}=\frac{(x+1)_{n}}{n !}, \quad \lim _{q \rightarrow 1} \tilde{\mathcal{P}}_{n}^{y}\left(q^{x}\right)=\frac{(x-y+1)_{n}}{n !}, \\
\lim _{q \rightarrow 1} \frac{Q_{q}^{\prime}\left(q^{x_{i}}\right)}{(q-1)^{d+N-1}}=Q^{\prime}\left(x_{i}\right), \quad \lim _{q \rightarrow 1} \frac{\Delta_{d}\left(q^{x_{1}}, \ldots, q^{x_{d}}\right)}{(q-1)^{d(d-1) / 2}}=\Delta_{d}\left(x_{1}, \ldots, x_{d}\right),
\end{gathered}
$$

and (due to Petrov[38])

$$
\begin{aligned}
\lim _{q \rightarrow 1} \frac{1}{(q-1)^{N-1}} \oint_{\Gamma_{\infty}} \frac{d z}{2 \pi \mathrm{i} z} \Phi_{q}\left(z^{-1}\right) \prod_{1}^{N-1}\left(z-q^{\rho_{r}-y}\right) \\
\quad=(N-n+1) ! \oint_{\Gamma_{\infty}} \frac{d z}{2 \pi \mathrm{i}(z-y)_{N-n+2}} \prod_{1}^{N-1}\left(z-\rho_{r}\right) .
\end{aligned}
$$

Proof: The limits are straightforward, except for Petrov's limit formula.

\section{An alternative integral representation for $h_{y-y_{j}}\left(1^{n}\right)$ and some determinantal identities}

Proposition 3.1 Given $d \geq 1$ and integers $\mathbf{y}=\left\{y_{d}<\cdots<y_{1}\right\}$ satisfying $N-1+y_{d}-y_{1} \geq 0$, and given the sum of the gaps $g:=y_{1}-y_{d}-d+1$ between these integers, the following holds, with the constant ${ }^{6} C_{N, d}=C_{N, d}^{\prime} C_{N, d}^{\prime \prime}$ and with $C_{N, d}^{\prime}=\prod_{j=1}^{d} \frac{1}{(N-j) !}$ and $C_{N, d}^{\prime \prime}=\frac{\Delta_{d}\left(y_{1}, \ldots, y_{d}\right)}{\prod_{j=1}^{d}(d-j) !}$ :

$$
\begin{aligned}
\operatorname{det} & \left(\frac{\left(x_{\alpha}-y_{\beta}+1\right)_{N-1}}{(N-1) !}\right)_{1 \leq \alpha, \beta \leq d} \\
& =C_{N, d} \Delta_{d}\left(x_{1}, \ldots, x_{d}\right) E_{g}^{(\mathbf{y})}\left(x_{1}, \ldots, x_{d}\right) \prod_{\alpha=1}^{d}\left(x_{\alpha}-y_{d}+1\right)_{N-\left(y_{1}-y_{d}+1\right)} .
\end{aligned}
$$

\footnotetext{
${ }^{6}$ The explicit form of the constant will never be used.
} 
where $E_{g}^{(\mathbf{y})}$ is a symmetric function of $x_{1}, \ldots, x_{d}$ with coefficients depending on the $y_{i}$ 's,

$$
E_{g}^{(\mathbf{y})}\left(x_{1}, \ldots, x_{d}\right)=\left(x_{1} \ldots x_{d}\right)^{g}+\text { lower order terms, }
$$

where the "lower order terms" refer to terms of total degree $<g d$, with degree in any $x_{i} \leq g$. For contiguous $\left(y_{1}, \ldots, y_{d}\right)=(d, \ldots, 1)$, we have $g=0$, and so the symmetric function $=1$ and $C_{N, d}^{\prime \prime}=1$.

Given an arbitrary choice of $k+\ell$ points $\left(x_{1}, \ldots, x_{k}, x_{1}^{\prime}, \ldots, x_{\ell}^{\prime}\right)$ in the set $\mathcal{L}$ (having $k+\ell$ points), and the associated power sum symmetric polynomial ${ }^{7}$ $t_{\alpha}(\mathcal{L}):=\sum_{i=1}^{k} x_{i}^{\alpha}+\sum_{i=1}^{\ell} x_{i}^{\prime \alpha}$, we have that any symmetric function $S\left(x_{1}, \ldots, x_{k}\right)$ (resp. $S\left(z ; x_{1}, \ldots, x_{k}\right)$ with some additional variable $z$ ) can be expressed in terms of a symmetric function in the complementary variables $x_{1}^{\prime}, \ldots, x_{\ell}^{\prime}$ (resp. $\left.z, x_{1}^{\prime}, \ldots, x_{\ell}^{\prime}\right)$. To be precise,

$$
\begin{aligned}
S\left(z, x_{1}, \ldots, x_{k}\right) & =F\left(z+\sum_{1}^{\alpha} x_{i}, \ldots, z^{\alpha}+\sum_{1}^{\alpha} x_{i}^{\alpha}\right) \\
& =F\left(z+t_{1}(\mathcal{L})-\sum_{1}^{\ell} x_{i}^{\prime}, \ldots, z^{\alpha}+t_{\alpha}(\mathcal{L})-\sum_{1}^{\ell} x_{i}^{\prime \alpha}\right) \\
& =: \widetilde{S}\left(z, x_{1}^{\prime}, \ldots, x_{\ell}^{\prime}\right),
\end{aligned}
$$

the latter being a new symmetric function, with coefficients depending polynomially on the variables $t_{\alpha}(\mathcal{L})$. Formula 44$)$ is also valid in the absence of the $z$-variable.

As an example, with one gap $(g=1)$, upon defining $\sigma_{i}(x)$ by $\prod_{1}^{d}\left(u+x_{i}\right)=$ $\sum_{i=0}^{d} \sigma_{i} u^{d-i}$

for

$$
\begin{aligned}
\mathbf{y}=(2<4<5<6): & E_{1}^{\mathbf{y}}(x)=\sigma_{4}-\sigma_{3}-\sigma_{2}+11 \sigma_{1}-49 \\
\mathbf{y}=(2<3<5<6<7): & E_{1}^{\mathbf{y}}(x)=\sigma_{5}-\frac{4}{5}\left(\sigma_{4}+\sigma_{3}\right)+4\left(\sigma_{2}+\sigma_{1}\right)-\frac{604}{5} .
\end{aligned}
$$

Proof: We give the proof for $\left(y_{d}, \ldots, y_{1}\right)=(1, \ldots, d)$. The determinant above has the general form $\operatorname{det}\left(\left(x_{\alpha}+\beta\right)_{N-1}\right)_{1 \leq \alpha, \beta \leq d}$. Each column of the matrix in the determinant $\operatorname{det}\left(\left(x_{\alpha}+\beta\right)_{N-1}\right)_{1 \leq \alpha, \beta \leq d}$ has the common factor $\left(x_{\alpha}+d\right)_{N-d}$, which, taken out, leaves us a determinant of the type below; by Lemma 3 in Krattenthaler [29], this determinant equals:

$$
\begin{aligned}
& \underset{1 \leq i, j \leq n}{\operatorname{det}}\left(\left(x_{i}+A_{n}\right)\left(x_{i}+A_{n-1}\right) \ldots\left(x_{i}+A_{j+1}\right)\left(x_{i}+B_{j}\right)\left(x_{i}+B_{j-1}\right) \ldots\left(x_{i}+B_{2}\right)\right) \\
& =\Delta_{n}(x) \prod_{2 \leq i, j \leq n}\left(B_{i}-A_{j}\right) .
\end{aligned}
$$

\footnotetext{
${ }^{7}$ Given variables $u_{1}, \ldots, u_{k}$, any symmetric polynomial $E(u)$ in these variables can be written as a polynomial in power sum symmetric polynomials $t_{\alpha}\left(u_{1}, \ldots, u_{k}\right)=\sum_{i=1}^{k} u_{i}^{\alpha}$ for integer $\alpha \geq 0$ with rational coefficients in the coefficients of the symmetric polynomial.
} 
In this expression $A_{j}=j-1, B_{j}=N+j-2$ for $2 \leq j \leq d$, from which it follows that $\prod_{2 \leq i, j \leq n}\left(B_{i}-A_{j}\right)=((N-1) !)^{d} \prod_{k=1}^{d}((N-k) !)^{-1}$, proving identity 42 .

Defining $B(y):=\max \left(y+n, y_{1}+1\right)-N$, we now define the contours

$$
\begin{aligned}
\gamma_{y} & :=\Gamma(y, y-1, \ldots, B(y)), \text { if } B(y) \leq y \\
& =\emptyset \quad, \text { if } B(y)>y,
\end{aligned}
$$

and the contour $\Gamma_{\tau}$, defined such that

$$
\gamma_{y}=\Gamma(y, y-1, \ldots, y+n-N) \backslash \Gamma_{\tau},
$$

given that a contour integral will be taken over a rational function with denominator equal to $(z-y)_{N-n+1}$. Then it is easily seen that

$$
\Gamma_{\tau}:=\left\{\begin{array}{l}
\Gamma\left(\min \left(y_{1}-N, y\right), \ldots, y+n-N\right), \text { if } \tau<0 \\
\emptyset, \text { if } \tau \geq 0
\end{array},\right.
$$

with $\Gamma_{\tau}$ containing exactly $-\tau>0$ points, if $y_{1}-N \leq y$ and $N-n+1$ points, if $y_{1}-N \geq y$, and none, when $\tau \geq 0$. This confirms definition 10.

We now state the following general Lemma, which will play a crucial role in the expression of the $\mathbb{K}$-kernel.

Proposition 3.2 Given $0 \leq n \leq N-1$ and integer points $\left(y_{1}>\cdots>y_{j}>\ldots\right)$ satisfying $0 \leq y_{1}-y_{j} \leq N-1$, the following holds, with the contours $\gamma_{y}$ and $\Gamma_{\tau}$ as in 45 and 47

$$
\begin{aligned}
h_{y-y_{j}}\left(1^{n}\right) & =\oint_{\Gamma_{0}} \frac{d u}{2 \pi \mathrm{i} u^{y-y_{j}+1}(1-u)^{n}} \\
& =\frac{(N-n) !}{(N-1) !} \oint_{\gamma_{y}} \frac{d z\left(z-y_{j}+1\right)_{N-1}}{2 \pi \mathrm{i}(z-y)_{N-n+1}} \\
& =\frac{(N-n) !}{(N-1) !}\left(\oint_{\Gamma(y, y-1, \ldots, y-N+n)}-\oint_{\Gamma_{\tau}}\right) \frac{d z\left(z-y_{j}+1\right)_{N-1}}{2 \pi \mathrm{i}(z-y)_{N-n+1}} .
\end{aligned}
$$

Proof: At first, notice that by Cauchy's theorem, the integral below, for $n \geq 0$, equals minus the sum of the residues at $z=1$ and $\infty$, with the residue at $\infty$ vanishing when $n+k-1 \geq 0$,

$$
\oint_{\Gamma_{0}} \frac{d z}{2 \pi \mathrm{i} z^{1+k}(1-z)^{n}}=\prod_{\ell=1}^{n-1} \frac{k+\ell}{\ell}-\operatorname{Res}_{z=\infty}=\frac{(k+1)_{n-1}}{(n-1) !}-\operatorname{Res}_{z=\infty} \mathbb{1}_{n+k \leq 0} .
$$


Consider the contour $\gamma_{y}$ as in 45). If $B(y)>y$, then we have $\max (y+$ $\left.n, y_{1}+1\right)>y+N$, implying that $y_{1}-N>y-1$, since $0 \leq n \leq N$, and thus $N \geq y_{1}-y_{j}+1>y-y_{j}+N$, and so $y-y_{j}+1 \leq 0$, implying that both sides of (48) vanish. In that case, since $\gamma_{y}=\emptyset$, we have that, in view of (46),

$$
\Gamma_{\tau}=\Gamma(y, y-1, \ldots, y+n-N) .
$$

So, we now consider $B(y) \leq y$. We have thus $\emptyset \neq\{y, y-1, \ldots, B(y)\} \subseteq\{y, y-$ $1, \ldots, y-N+n\}$. Then, since $y-B(y)=N-n-\max \left(y_{1}+1-y-n, 0\right)$, we have for the right hand side of (48), upon evaluating by residues and upon using the change of variables $\ell=y-k$ and $i=y-j$ in $\stackrel{*}{=}$,

$$
\begin{aligned}
& \frac{(N-n) !}{(N-1) !} \sum_{\ell=B(y)}^{y} \frac{\left(\ell-y_{j}+1\right) \ldots\left(\ell-y_{j}+N-1\right)}{\prod_{\substack{i=y-N+n \\
i \neq \ell}}^{y}(\ell-i)} \\
& \quad \stackrel{*}{=} \frac{(N-n) !}{(N-1) !} \sum_{k=0}^{y-B(y)} \frac{\left(y-y_{j}-k+1\right) \ldots\left(y-y_{j}-k+N-1\right)}{\prod_{\substack{j=0 \\
j \neq k}}^{N-n}(j-k)} \\
& =\frac{(N-n) !}{(N-1) !} \sum_{k=0}^{y-B(y)} \frac{(-1)^{k}\left(y-y_{j}-k+1\right)_{N-1}}{k !(N-n-k) !} \\
& =\sum_{k=0}^{N-n-\max \left(0, y_{1}+1-y-n\right)}(-1)^{k}\left(\begin{array}{c}
N-n \\
k
\end{array}\right) \frac{\left(y-y_{j}-k+1\right)_{N-1}}{(N-1) !} \\
& \stackrel{* * *}{=} \sum_{k=0}^{N-n-\max \left(0, y_{1}+1-y-n\right)}(-1)^{k}\left(\begin{array}{c}
N-n \\
k
\end{array}\right) \oint_{\Gamma_{0}} \frac{d z}{z^{y-y_{j}-k+1}(1-z)^{N}},
\end{aligned}
$$

provided $y-y_{j}-k+N-1 \geq 0(*)$ holds for all $0 \leq k \leq N-n-\max \left(0, y_{1}+1-y-n\right)$ in the expression on the right hand side of $\stackrel{* *}{=}$. It suffices to check this for the largest value $k=N-n-\max \left(0, y_{1}+1-y-n\right)$. Indeed, this is so:

(i) If $y+n \geq y_{1}+1$, then $k=N-n$ and $y-y_{j}-k+N-1=y+n-y_{j}-1 \geq$ $y_{1}-y_{j} \geq 0$.

(ii) If $y+n<y_{1}+1$, then $k=N+y-y_{1}-1$ and so $y-y_{j}-k+N-1=y_{1}-y_{j} \geq 0$; this checks the inequality $(*)$ above. For this same case, namely $y+n<y_{1}+1$, and for $N-n-\left(y_{1}+1-y-n\right)+1 \leq k \leq N-n$, we also have that the integrand in the last expression (50) has no residue at $z=0$, because the $z$-exponent in its 
denominator equals

$y-y_{j}-k+1 \leq y-y_{j}-\left(N-n-\left(y_{1}+1-y-n\right)+1\right)+1=y_{1}-y_{j}-N+1 \leq 0$,

using the inequality in the statement.

Therefore, in the last expression in (50) the sum can be extended to $0 \leq k \leq N-n$ and so the final expression reads

$$
\begin{aligned}
\sum_{k=0}^{N-n}(-1)^{k}\left(\begin{array}{c}
N-n \\
k
\end{array}\right) \oint_{\Gamma_{0}} \frac{z^{k} d z}{2 \pi \mathrm{i} z^{y-y_{j}+1}(1-z)^{N}} & =\oint_{\Gamma_{0}} \frac{d z(1-z)^{N-n}}{2 \pi \mathrm{i} z^{y-y_{j}+1}(1-z)^{N}} \\
& =\oint_{\Gamma_{0}} \frac{d z}{2 \pi \mathrm{i} z^{y-y_{j}+1}(1-z)^{n}}
\end{aligned}
$$

The last formula of (48) follows from formula (46), thus ending the proof of Proposition 3.2 .

Both Propositions will be applied to certain determinants, which will come up later, and which depend on the geometry of $\mathbf{P}$. In the corollary below, the $u_{1}, \ldots, u_{d}$ are arbitrary complex variables. Referring to the model $\mathbf{P}$, remember $\mathbf{y}_{\text {cut }}=\left\{y_{1}>\cdots>y_{d}\right\}$ are the integer points in the \{lower-cuts $\} \cap\{n=0\}$ and $P(z)$ is as in (2). We define three expressions, which will play an important role in Section 7:

$$
\begin{aligned}
\Delta_{d}^{\left(\mathbf{y}_{\text {cut }}\right)}\left(u_{1}, \ldots, u_{d}\right) & :=\operatorname{det}\left(\frac{\left(u_{\alpha}-y_{\beta}+1\right)_{N-1}}{(N-1) !}\right)_{1 \leq \alpha, \beta \leq d} \\
\widetilde{\Delta}_{d}^{\left(\mathbf{y}_{\text {cut }}\right)}\left(w ; u_{2}, \ldots, u_{d}\right) & :=\operatorname{det}\left(\begin{array}{c}
w^{y_{1}} \\
\left(\frac{\left(u_{\alpha}-y_{\beta}+1\right)_{N-1}}{(N-1) !}\right)_{\substack{2 \leq \alpha \leq d \\
1 \leq \beta \leq d}}^{y_{d}}
\end{array}\right) \\
\widetilde{\Delta}_{d, n}^{\left(\mathbf{y}_{\text {cut }}\right)}\left(y ; u_{2}, \ldots, u_{d}\right) & :=\oint_{\Gamma_{0}} \frac{d w}{2 \pi \mathrm{i} w^{y+1}(1-w)^{n}} \widetilde{\Delta}_{d}^{\left(\mathbf{y}_{\mathrm{cut}}\right)\left(w ; u_{2}, \ldots, u_{d}\right)} \\
& =\operatorname{det}\left(\begin{array}{c}
h_{y-y_{1}\left(1^{n}\right)} \\
\left(\frac{\left(u_{\alpha}-y_{\beta}+1\right)_{N-1}}{(N-1) !}\right)_{\substack{2 \leq \alpha \leq d \\
1 \leq \beta \leq d}}\left(1^{n}\right)
\end{array}\right) .
\end{aligned}
$$

We now apply formula (42) (or the first formula (51)) to the (integer) points $y_{1}>\cdots>y_{d}$ in the lower cuts, thus leading to the symmetric function $E_{g}^{\left(\mathbf{y}_{\text {cut }}\right)}\left(u_{1}, \ldots, u_{d}\right)$, as in 42 . 
Corollary 3.3 The following identities hold for the determinantal expressions in (51), with $C_{N, d}$ as in (42), $P(u)$ as in (2), and the contour $\Gamma_{\tau}$ as in (47):

$$
\Delta_{d}^{\left(\mathbf{y}_{c u t}\right)}\left(u_{1}, \ldots, u_{d}\right)=C_{N, d} E_{g}^{\left(\mathbf{y}_{c u t}\right)}(u) \Delta_{d}(u) \prod_{\alpha=1}^{d} P\left(u_{\alpha}\right),
$$

and

$$
\begin{aligned}
\widetilde{\Delta}_{d, n}^{\left(\mathbf{y}_{\text {cut }}\right)}\left(y ; u_{2}, \ldots, u_{d}\right)= & C_{N, d}\left(\oint_{\Gamma(y, y-1, \ldots, y-N+n)}-\oint_{\Gamma_{\tau}}\right) \frac{(N-n) ! d z}{2 \pi \mathrm{i}(z-y)_{N-n+1}} \\
& \times E_{g}^{\left(\mathbf{y}_{c u t}\right)}\left(z, u_{2}, \ldots, u_{d}\right) \Delta_{d}\left(z, u_{2}, \ldots, u_{d}\right) P(z) \prod_{\alpha=2}^{d} P\left(u_{\alpha}\right) .
\end{aligned}
$$

Proof: The first identity (52) follows at once from Proposition 3.1. The second identity (53) follows from moving the $w$-integral to the first row of $\widetilde{\Delta}_{d}^{\left(\mathbf{y}_{\text {cut }}\right)}\left(w, \ldots, u_{d}\right)$, using Proposition 3.2 :

$$
\begin{aligned}
& \widetilde{\Delta}_{d, n}^{\left(\mathbf{y}_{\text {cut }}\right)}\left(y ; u_{2}, \ldots, u_{d}\right) \\
& =\left.\oint_{\gamma_{y}} \frac{(N-n) ! d z}{2 \pi \mathrm{i}(z-y)_{N-n+1}} \operatorname{det}\left(\frac{\left(u_{\alpha}-y_{\beta}+1\right)_{N-1}}{(N-1) !}\right)_{1 \leq \alpha, \beta \leq d}\right|_{u_{1}=z} \\
& =\oint_{\gamma_{y}} \frac{(N-n) ! d z}{2 \pi \mathrm{i}(z-y)_{N-n+1}} \Delta_{d}^{\left(\mathbf{y}_{\text {cut }}\right)}\left(z, u_{2}, \ldots, u_{d}\right) .
\end{aligned}
$$

Finally using the first identity 52 , applied to $\Delta_{d}^{\left(\mathbf{y}_{\text {cut }}\right)}\left(z, u_{2}, \ldots, u_{d}\right)$ ends the proof of Corollary 3.3 .

The next lemma deals with identities involving Vandermonde's with certain rows removed. For $\mathbf{x} \in \mathbb{R}^{m}$ and $\mathbf{y} \in \mathbb{R}^{n}$, define the Vandermonde

$$
\begin{aligned}
& \Delta_{m}(\mathbf{x})=\operatorname{det}\left(\begin{array}{ccc}
x_{1}^{m-1} & \ldots & x_{1}^{0} \\
\vdots & \ldots & \vdots \\
x_{m}^{m-1} & \ldots & x_{m}^{0}
\end{array}\right)=\prod_{1 \leq i<j \leq m}\left(x_{i}-x_{j}\right) \\
& \Delta_{m}^{\hat{k}}(\mathbf{x})=\operatorname{det}\left(\begin{array}{cccccc}
x_{1}^{m} & \ldots & x_{1}^{k+1} & x_{1}^{k-1} & \ldots & x_{1}^{0} \\
\vdots & & \vdots & \vdots & & \vdots \\
x_{m}^{m} & \ldots & x_{m}^{k+1} & x_{m}^{k-1} & \ldots & x_{m}^{0}
\end{array}\right)=\Delta_{m}(\mathbf{x}) e_{m-k}(\mathbf{x}),
\end{aligned}
$$

where $\hat{k}$ refers to removing the column containing the $k$ th power. Also

$$
e_{k}(\mathbf{y})=\sum_{i_{1}<i_{2}<\cdots<i_{k}} y_{i_{1}} \ldots y_{i_{k}} .
$$

We will need the following Lemma: 
Lemma 3.4 Then we have (here $\left.Q(z):=\prod_{1}^{m}\left(z-x_{i}\right) \prod_{1}^{n}\left(z-y_{i}\right)\right)$

$$
\frac{\Delta_{n}^{n-k}(\mathbf{y})}{\Delta_{n+m}(\mathbf{x}, \mathbf{y})}=(-1)^{m(m-1) / 2} \frac{\Delta_{m}(\mathbf{x})}{\prod_{1}^{m} Q^{\prime}\left(x_{\ell}\right)} e_{k}(\mathbf{y}),
$$

and so in particular, setting $k=0$,

$$
\frac{\Delta_{n}(\mathbf{y})}{\Delta_{n+m}(\mathbf{x}, \mathbf{y})}=(-1)^{m(m-1) / 2} \frac{\Delta_{m}(\mathbf{x})}{\prod_{1}^{m} Q^{\prime}\left(x_{\ell}\right)} .
$$

The following symmetric function, with variables removed, has the integral representation: (with $Q_{q}$ defined in (38))

$$
\begin{aligned}
(-1)^{r-1} e_{r-1} & \left(q_{1}, \ldots, \hat{q}_{k}, \hat{q}_{i_{1}}, \ldots, \hat{q}_{i_{d}}, \ldots, q_{d+N}\right) \\
= & \oint_{\Gamma_{\infty}} \frac{d z}{2 \pi \mathrm{i} z^{N-r+1}} \frac{Q_{q}(z)}{\left(z-q_{k}\right) \prod_{\ell=1}^{d}\left(z-q_{i_{\ell}}\right)},
\end{aligned}
$$

Proof: The first relation is shown by multiplication by $z^{n-k}(-1)^{k}$ and summing from $k=0$ to $n$, which leads to an obvious identity . The second is straightforward and the third is just the residue Theorem.

\section{From Karlin-McGregor to the $\mathbb{K}_{q}$-kernel}

Since the $\mathbb{K}$-process of red dots has a nonintersecting paths description, one expects to have a Karlin-McGregor formula for the $q$-dependent probability measure $\mathbb{P}_{q}$, as in $(26)$ above. This will be shown in Proposition 4.1. Then in Proposition 4.2 , we will obtain a kernel by adapting the arguments in Borodin-FerrariPrähofer [7] and in Borodin-Rains [9] to these new circumstances.

We first need some notation. Remember from (22) the interlacing pattern $\mathbb{1}_{x^{(m-1)} \prec x^{(m)}}$ for $1 \leq m \leq N$. To the left of each sequence $x^{(m-1)}$, we add an extra point, a so-called virtual point $x_{d+m}^{(m-1)}=$ virt; it can be thought of as a point at $-\infty$. Then we define for $x \in\{$ virt $\} \cup \mathbb{Z}, z, k \in \mathbb{Z}$ and $k \geq 0$, the following functions, where the matrix $H$ was defined in (56):

$$
\begin{aligned}
& \chi_{i}(z):=\mathbb{1}_{z=y_{i}} \text {, for } 1 \leq i \leq d \\
& \varphi_{k}(x, z):=q^{(k-1)(z-x)} \mathbb{1}_{x \in \mathbb{Z}} \mathbb{1}_{x \leq z}+q^{(k-1) z} \mathbb{1}_{x=\text { virt }} \\
& =\frac{1}{2 \pi \mathrm{i}} \oint_{\Gamma_{0}} \frac{d u}{1-u q^{k-1}}\left(\frac{1}{u^{z-x+1}}\right)+q^{(k-1) z} \mathbb{1}_{x=\text { virt }} \\
& =h_{z-x}\left(q^{k-1}\right)+q^{(k-1) z} \mathbb{1}_{x=\text { virt }} \\
& \psi_{i}(z):=\sum_{j=1}^{N+d}\left(H^{-1}\right)_{i j} \mathbb{1}_{z=x_{j}} \text {, for } 1 \leq i \leq d+N \text {. }
\end{aligned}
$$


So, in particular we have $\varphi_{k}($ virt, $z)=q^{(k-1) z}$.

Proposition 4.1 The probability (26) can be written as a product of $N+2$ determinants

$$
\begin{aligned}
& \mathbb{P}_{q}\left(\nu^{(0)}, \nu^{(1)}, \ldots \nu^{(N)}, \text { such that } \mu=\nu^{(0)} \subset \nu^{(1)} \subset \ldots \subset \nu^{(N)}=\lambda\right) \\
& =C_{N, d, q} \operatorname{det}\left(\chi_{i}\left(x_{j}^{(0)}\right)\right)_{1 \leq i, j \leq d} \\
& \quad \times \prod_{m=1}^{N} \operatorname{det}\left(\varphi_{d+m}\left(x_{i}^{(m-1)}, x_{j}^{(m)}\right)\right)_{1 \leq i, j \leq d+m} \operatorname{det}\left(\psi_{i}\left(x_{j}^{(N)}\right)\right)_{1 \leq i, j \leq N+d},
\end{aligned}
$$

with

$$
C_{N, d, q}=q^{d N(d+N)+\frac{1}{3} N\left(N^{2}-1\right)} .
$$

Define the following $*$-operation between two real or complex functions $f(k)$ and $g(k)$ defined for $k \in \mathbb{Z}$ :

$$
f * g:=\sum_{k \in \mathbb{Z}} f(k) g(k)
$$

e.g., for functions $\varphi_{k}$ as above, the operation $*$ means a convolution $\left(\varphi_{k} *\right.$ $\left.\varphi_{k+1}\right)(x, y)=\varphi_{k}(x, \circ) * \varphi_{k+1}(\circ, y)=\sum_{z \in \mathbb{Z}} \varphi_{k}(x, z) \varphi_{k+1}(z, y)$. Subsequently, define

$$
\varphi^{n, m+1}(x, y):=\left(\varphi_{n} * \ldots * \varphi_{m}\right)(x, y) \delta_{n \leq m} .
$$

The functions $\psi_{j}$, as in (56), have a natural extension in terms of the geometry of $\mathbf{P}$ : namely, for $0 \leq m \leq N-1$ and $1 \leq k \leq d+N$, define:

$\psi_{k}^{(m+1)}(x):=\varphi_{d+m+1}(x, \bullet) * \cdots * \varphi_{d+N}(\bullet, \circ) * \psi_{k}(\circ)=\varphi^{d+m+1, d+N+1}(x, \circ) * \psi_{k}(\circ)$

and for $m=N$ we have $\psi_{j}^{(N+1)}=\psi_{j}$.

Proposition 4.2 The $\mathbb{K}_{q}$-point-process is determinantal with kernel, for $0 \leq$ $m, n \leq N$, given by

$$
\begin{aligned}
& \mathbb{K}_{q}(m, x ; n, y) \\
& =-\varphi_{d+m+1} * \ldots * \varphi_{d+n}(x, y) \\
& \quad+\sum_{\substack{1 \leq k \leq d+N \\
1 \leq \ell \leq d}} \psi_{k}^{(m+1)}(x)\left(M^{-1}\right)_{k \ell}\left(\chi_{\ell} * \varphi^{d+1, d+1+n}\right)(y) \\
& \quad+\sum_{\substack{1 \leq k \leq d+N \\
d+1 \leq \ell \leq d+n}} \psi_{k}^{(m+1)}(x)\left(M^{-1}\right)_{k \ell}\left(\varphi_{\ell}(\text { virt }, \bullet) * \varphi^{\ell+1, d+1+n}(\bullet, y)\right),
\end{aligned}
$$

\footnotetext{
${ }^{8}$ The $\ell$-summation in the third term of 61 goes up to $\ell=d+n$; that term contains $\varphi^{d+n+1, d+n+1}$, which we declare $=1$.
} 
where for $1 \leq i, j \leq N+d$ :

$$
M_{k \ell}:= \begin{cases}\chi_{k}(\circ) * \psi_{\ell}^{(1)}(\circ), & 1 \leq k \leq d \\ \varphi_{k}(\text { virt }, \circ) * \psi_{\ell}^{(k+1-d)}(\circ), & d+1 \leq k \leq d+N .\end{cases}
$$

Before giving the proof of Propositions 4.1 and 4.2 , the following Lemma will be needed:

Lemma 4.3 The following determinantal identities hold: for $1 \leq m \leq N$,

$$
\begin{aligned}
& \operatorname{det}\left(\chi_{i}\left(x_{j}^{(0)}\right)\right)_{1 \leq i, j \leq d}=\prod_{1}^{d} \mathbb{1}_{x_{j}^{(0)}=y_{j}} \\
& \left.\operatorname{det}\left(\varphi_{k}\left(x_{i}^{(m-1)}, x_{j}^{(m)}\right)\right)_{1 \leq i, j \leq d+m}=q^{(k-1)\left(\left|x^{(m)}\right|-\left|x^{(m-1)}\right|\right)_{1}} \mathbb{1}_{x^{(m-1)} \prec x^{(m)}}\right)_{1 \leq i, j \leq N+d} \prod_{j=1}^{d+N} \mathbb{1}_{x_{j}^{(N)}=x_{j}} . \\
& \operatorname{det}\left(\psi_{i}\left(x_{j}^{(N)}\right)\right)_{1 \leq i, j \leq N+d}=\operatorname{det}\left(h_{x_{i}-y_{j}}\left(q^{d}, \ldots, q^{d+N-1}\right)\right.
\end{aligned}
$$

Remembering the *-operation (58), we have the following convolution properties for $x, y \in \mathbb{Z}$, with the last identity valid for $1 \leq k \leq d+N, 1 \leq m \leq N$ :

$$
\begin{gathered}
\varphi^{n+1, m+1}(x, y)=\left(\varphi_{n+1} * \ldots * \varphi_{m}\right)(x, y)=h_{y-x}\left(q^{n}, \ldots, q^{m-1}\right) \mathbb{1}_{n<m} \\
=\frac{1}{2 \pi \mathrm{i}} \oint_{\Gamma_{0}} \frac{d z}{z^{y-x+1}} \prod_{\ell=n}^{m-1} \frac{1}{1-u q^{\ell}} \mathbb{1}_{n<m} \\
\chi_{\ell} *\left(\varphi_{n+1} * \ldots * \varphi_{m}\right)(y)=h_{y-y_{\ell}}\left(q^{n}, \ldots, q^{m-1}\right) \mathbb{1}_{n<m} \\
\varphi_{n}(\text { virt }, \cdot) *\left(\varphi_{n+1} * \ldots * \varphi_{m}\right)(\cdot, y)=\mathbb{1}_{n<m} \frac{q^{(n-1) y}}{(1-q) \ldots\left(1-q^{m-n}\right)} \\
\varphi_{n}(v i r t, \cdot) *\left(\varphi_{n+1} * \ldots * \varphi_{m}\right)(\cdot, \circ) * \psi_{j}(\circ)= \\
\frac{1}{(1-q) \ldots\left(1-q^{m-n}\right)} \sum_{\ell=1}^{N+d}\left(H^{-1}\right)_{j \ell} q^{(n-1) x_{\ell}}, \\
\varphi^{n+1, m+1}(x, \circ) * \psi_{j}(\circ)=\sum_{\ell=1}^{d+N}\left(H^{-1}\right)_{j \ell} h_{x_{\ell}-x}\left(q^{n}, \ldots, q^{m-1}\right) \\
\chi_{i}(\cdot) * \varphi^{n+1, m+1}(\cdot, \circ) * \psi_{j}(\circ)=\sum_{\ell=1}^{d+N}\left(H^{-1}\right)_{j \ell} h_{x_{\ell}-y_{i}}\left(q^{n}, \ldots, q^{m-1}\right) . \\
\psi_{k}^{(m)}(x)=\sum_{\ell=1}^{d+N}\left(H^{-1}\right)_{k \ell} h_{x_{\ell}-x}\left(q^{d+m-1}, \ldots, q^{d+N-1}\right) .
\end{gathered}
$$


Proof: The proof of the first three determinantal identities is straightforward; for instance,

$$
\begin{aligned}
\operatorname{det} & \left(\varphi_{k}\left(x_{i}^{(m-1)}, x_{j}^{(m)}\right)\right)_{1 \leq i, j \leq d+m} \\
& =q^{(k-1)\left(\sum_{i=1}^{d+m} x_{i}^{(m)}-\sum_{i=1}^{d+m-1} x_{i}^{(m-1)}\right)} \operatorname{det}\left(\mathbb{1}_{x_{i}^{(m-1)} \leq x_{j}^{(m)}}\right)_{1 \leq i, j \leq d+m} \\
& =q^{(k-1)\left(\left|x^{(m)}\right|-\left|x^{(m-1)}\right|\right)} \mathbb{1}_{x^{(m-1)} \prec x^{(m)}},
\end{aligned}
$$

taking into account the fact that the last row of the matrix above is given by $\left(q^{(k-1) x_{1}^{(m)}}, \ldots, q^{(k-1) x_{d+m}^{(m)}}\right)$.

The convolution properties (64) follow from the identity (29), applied to the partition $\lambda=(r, 0,0, \ldots)$ and $\mu=(s, 0,0, \ldots)$ with $r>s$, i.e., $\mu \subset \lambda$. Indeed one has:

$$
s_{\lambda}(x)=h_{r}(x), \quad s_{\lambda \backslash \mu}(x)=h_{r-s}(x),
$$

and so, setting $\nu=(\alpha, 0,0, \ldots)$, one has

$$
h_{r-\alpha}(x, y)=\sum_{\alpha \in \mathbb{Z}} h_{r-\alpha}(x) h_{\alpha-s}(y)
$$


Another example of proof is:

$$
\begin{aligned}
\varphi_{n}(\operatorname{virt}, \cdot) *\left(\varphi_{n+1}\right. & \left.* \ldots * \varphi_{m}\right)(\cdot, \circ) * \psi_{j}(\circ) \\
& =\sum_{z \in \mathbb{Z}} \frac{q^{(n-1) z}}{(1-q) \ldots\left(1-q^{m-n}\right)} \sum_{\ell=1}^{N+d}\left(H^{-1}\right)_{j \ell} \mathbb{1}_{z=x_{\ell}} \\
& =\frac{1}{(1-q) \ldots\left(1-q^{m-n}\right)} \sum_{\ell=1}^{N+d}\left(H^{-1}\right)_{j \ell} q^{(n-1) x_{\ell}},
\end{aligned}
$$

ending the proof of Lemma 4.3 .

Proof of Proposition 4.1. The denominator of the probability $(26)$ can be expressed as a determinant, using formula (36). Also from the second formula (63), it follows that

$$
\begin{aligned}
\prod_{m=1}^{N} \operatorname{det} & \left(\varphi_{d+m}\left(x_{i}^{(m-1)}, x_{j}^{(m)}\right)\right)_{1 \leq i, j \leq d+m} \\
& =q^{(N-1)\left|x^{(N)}\right|+d\left(\left|x^{(N)}\right|-\left|x^{(0)}\right|\right)} q^{-\sum_{1}^{N-1}\left|x^{(i)}\right|} \mathbb{1}_{x^{(0)} \prec x^{(1)} \prec \ldots \prec x^{(N)}} \\
& =q^{(d+N-1)|x|-d|y|)} q^{-\sum_{1}^{N-1}\left|x^{(i)}\right|} \mathbb{1}_{x^{(0)} \prec x^{(1)} \prec \ldots \prec x^{(N)}}
\end{aligned}
$$

and then using the two remaining formulas of $(63)$, one checks 9 :

$$
\begin{aligned}
& \mathbb{P}\left(\nu^{(0)}, \nu^{(2)}, \ldots \nu^{(N)}, \text { such that } \mu=\nu^{(0)} \subset \nu^{(1)} \subset \ldots \subset \nu^{(N)}=\lambda\right) \\
& =\frac{\left(q^{-1}\right)^{\sum_{i=2}^{N}\left|\nu^{(i-1)}\right|-(N-1)\left|\nu^{(0)}\right|}}{s_{\lambda \backslash \mu}\left(q^{1-N}, q^{2-N}, \ldots, q^{-1}, q^{0}\right)} \mathbb{1}_{\nu^{(0)} \subset \nu^{(1)} \subset \ldots \subset \nu^{(N)} \mathbb{1}_{\nu^{(0)}=\mu} \mathbb{1}_{\nu^{(N)}=\lambda}} \\
& =q^{N(d+N-1)(d+(N+1) / 2)} q^{-\sum_{1}^{N}(d+i-1)(d+i) / 2+d(d+1) / 2} q^{(N-1) d(d+1) / 2} \\
& \quad \frac{q^{(d+N-1)(|x|-|y|)}\left(q^{-1}\right)^{\sum_{i=1}^{N-1}\left|x^{(i)}\right|-(N-1)|y|}}{\operatorname{det}\left(h_{x_{i}-y_{j}}\left(q^{d}, \ldots, q^{d+N-1}\right)\right)_{1 \leq i, j \leq N+d}} \mathbb{1}_{\nu^{(0)} \subset \nu^{(1)} \subset \ldots \subset \nu^{(N)}} \mathbb{1}_{\nu^{(0)}=\mu} \mathbb{1}_{\nu^{(N)}=\lambda} \\
& =C_{N, d, q} \frac{q^{(d+N-1)|x|-d|y|}\left(q^{-1}\right)^{\sum_{i=1}^{N-1}\left|x^{(i)}\right|}}{\operatorname{det}\left(h_{x_{i}-y_{j}}\left(q^{d}, \ldots, q^{d+N-1}\right)\right)_{1 \leq i, j \leq N+d}} \mathbb{1}_{\nu^{(0)} \subset \nu^{(1)} \subset \ldots \subset \nu^{(N)} \mathbb{1}_{\nu^{(0)}=\mu} \mathbb{1}_{\nu^{(N)}=\lambda}} \\
& =C_{N, d, q} \operatorname{det}\left(\chi_{i}\left(x_{j}^{(0)}\right)\right)_{1 \leq i, j \leq d} \\
& \quad \times \prod_{m=1}^{N} \operatorname{det}\left(\varphi_{d+m}\left(x_{i}^{(m-1)}, x_{j}^{(m)}\right)\right)_{1 \leq i, j \leq d+m} \operatorname{det}\left(\psi_{i}\left(x_{j}^{(N)}\right)\right)_{1 \leq i, j \leq N+d},
\end{aligned}
$$

establishing the Karlin-MacGregor formula of Proposition 4.1.

Proof of Proposition 4.2, The technology explained in [7, 9] will now be adapted to these new circumstances. Given a set $\mathfrak{X}$, let $L$ be a positive-definite $|\mathfrak{X}| \times|\mathfrak{X}|$ matrix with rows and columns parametrized by the points $x \in \mathfrak{X}$, with $L_{X}$ a $X \times X$-submatrix with rows and columns parametrized by $x \in X$. A random point process $\left(L\right.$-ensemble) on $\mathfrak{X}$ will be defined by the probability $\mathbb{P}(X)=\frac{\operatorname{det}\left(L_{X}\right)}{\operatorname{det}(\mathbb{1}+L)}$

\footnotetext{
${ }^{9}$ One uses $\sum_{1}^{N}(d+i-1)(d+i) / 2=\frac{1}{2}\left(d N(d+N)+\frac{1}{3} N\left(N^{2}-1\right)\right)$
} 
for $X \in \mathfrak{X}$. It is determinantal with kernel $K=L(\mathbb{1}+L)^{-1}$; that means that the correlation $\rho(Y)=\mathbb{P}(X \subset \mathfrak{X} \mid Y \subset X)=\operatorname{det} K_{Y}$. Given a decomposition $\mathfrak{X}=\mathfrak{Y} \cup \mathfrak{Y}^{c}$, with $\mathfrak{Y} \neq \emptyset$ and with corresponding block matrix $\mathbb{1}_{\mathfrak{Y}}$, the conditional $L$-ensemble process on $\mathfrak{Y}$ is defined by $\mathbb{P}(Y)=\frac{\operatorname{det} L_{Y \cup \mathfrak{Y} c}}{\operatorname{det}\left(\mathbb{1}_{\mathfrak{Y}}+L\right)}$ and is determinantal with correlation kernel $K=\mathbb{1}_{\mathfrak{Y}}-\left(\mathbb{1}_{\mathfrak{Y}}+L\right)_{\mathfrak{Y} \times \mathfrak{Y}}^{-1}$.

This is now applied to $\mathfrak{X}=\mathfrak{Y} \cup \mathfrak{Y}^{c}=:\left(\mathfrak{X}^{(0)} \cup \cdots \cup \mathfrak{X}^{(N)}\right) \cup$ $\left\{1,2, \ldots, d, x_{d+1}^{(0)}, \ldots, x_{d+N}^{(N-1)}\right\}$, where $\mathfrak{X}^{(k)}=\mathbb{Z}$ are all the point configurations at level $0 \leq k \leq N$ and the $x_{d+k+1}^{(k)}=$ virt are virtual variables, playing the role of point at $-\infty$ for each set $\mathfrak{X}^{(k)}$ for $0 \leq k \leq N-1$; .

We now apply this set-up to the specific situation of the paper, although this is completely general. Indeed, we now define (infinite) matrices in terms of the functions appearing in the Karlin-McGregor formula (61), where $\Phi^{(d)}$ contains the initial condition (points not in $\mathbf{P}$ at level $y=0$, in particular the lower-cut) for the red dots, where the $T^{k-1, k}$ are the transition functions from one level to another and where $\Psi^{(d+N)}$ contains the final condition for the red dots (points not in $\mathbf{P}$ at level $y=N$, in particular the upper-cuts):

$$
\begin{gathered}
\text { - } \Phi^{(d)}=\{1, \ldots, d\} \times \mathfrak{X}^{(0)} \text {-matrix, with }\left(\Phi^{(d)}\right)\left(i, x^{(0)}\right)=\chi_{i}\left(x^{(0)}\right) \\
\text { - } T^{k-1, k}=\left(\mathfrak{X}^{(k-1)} \cup\left\{x_{d+k}^{(k-1)}\right\}\right) \times \mathfrak{X}^{(k)} \text {-matrix, for } 1 \leq k \leq N \\
\quad \text { with } T^{k-1, k}\left(x^{(k-1)}, x^{(k)}\right)=\varphi_{d+k}\left(x^{(k-1)}, x^{(k)}\right) \\
\text { - } \Psi^{(d+N)}=\left(\mathfrak{X}^{(N)} \times\{1, \ldots, d+N\}\right) \text {-matrix, } \\
\text { with } \Psi^{(d+N)}\left(x^{(N)}, j\right):=\psi_{j}\left(x^{N}\right) .
\end{gathered}
$$

The conditional $L$-ensemble on $\mathfrak{X}$ with $\mathfrak{Y}$ as above is given by the block matrix, where each block has infinite size. The only nonzero determinants of the form $\operatorname{det} L_{Y \cup \mathfrak{Y}^{c}}$ appearing in $\mathbb{P}(Y)$ above are suitable finite minors of this matrix $L$ below. These lead exactly to the Karlin-McGregor formula (57), as is easily seen by picking appropriate rows and columns according to the coordinates on top and to the left of the matrix $L$ below :

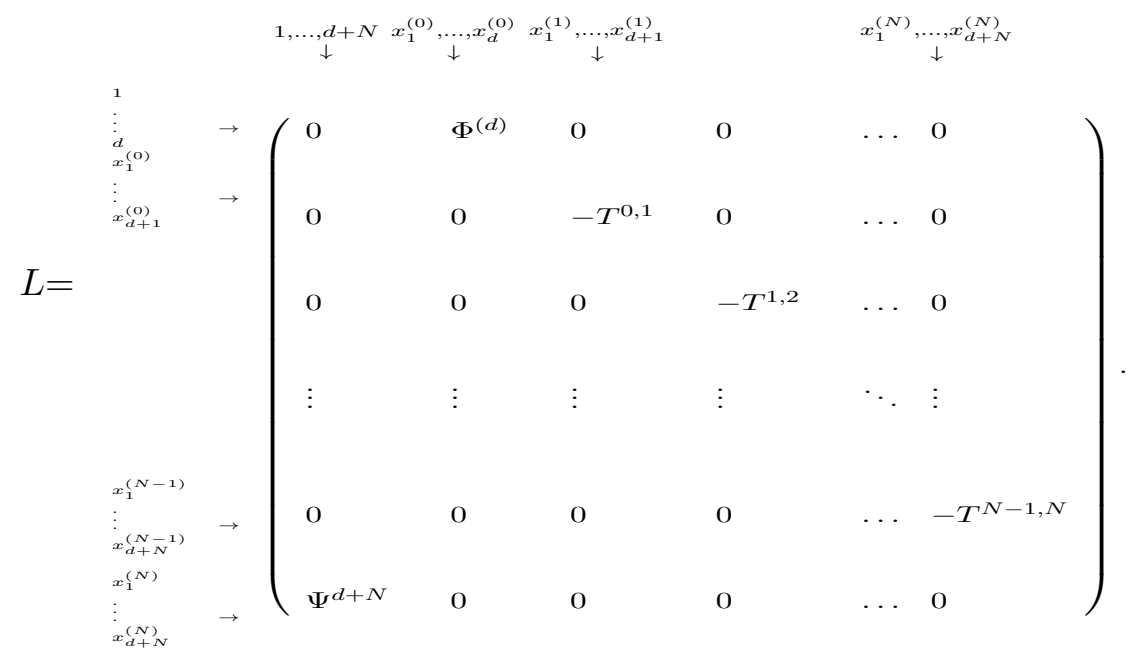


We now move rows and columns of $L$ in order to write the matrix as a block $\left(\mathfrak{Y}^{c} \otimes \mathfrak{Y}\right)$-matrix $L_{\mathfrak{Y}^{c} \otimes \mathfrak{Y}}$. Indeed, move the rows corresponding to the virtual variables

$$
x_{d+1}^{(0)}, x_{d+2}^{(1)}, \ldots, x_{d+k}^{(k-1)}, \ldots, x_{d+N}^{(N-1)}
$$

respectively to the $d+1, d+2, \ldots, d+k, \ldots, d+N^{\text {th }}$ row, yielding the matrix below, where the $\mathfrak{X}^{(k-1)} \times \mathfrak{X}^{(k)}$-matrices $W_{k-1, k}$ are the matrices $T^{k-1, k}$ with the last row removed,

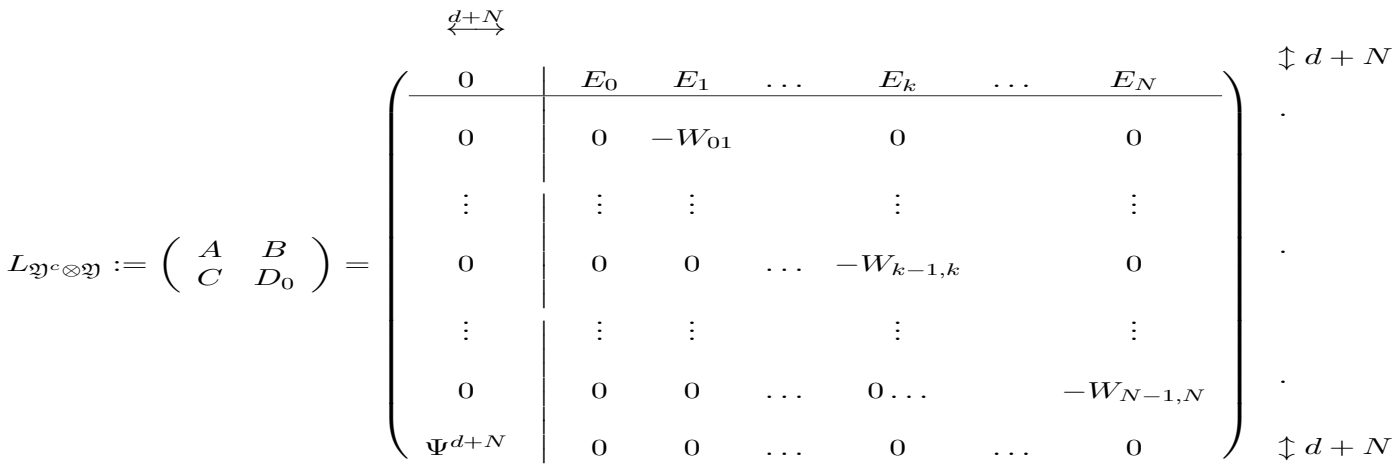

and

$$
\mathbb{1}_{\mathfrak{Y}}=\left(\begin{array}{ll}
0 & 0 \\
0 & \mathbb{1}
\end{array}\right)
$$

with

$$
\begin{gathered}
A=0, \quad B:=\left(E_{0}, \ldots, E_{N}\right), \\
D_{0}:=\left(\begin{array}{ccccc}
0 & -W_{01} & 0 & \cdots & 0 \\
0 & 0 & -W_{12} & \cdots & 0 \\
\vdots & \vdots & \vdots & & \vdots \\
0 & 0 & 0 & \cdots & -W_{N-1, N} \\
0 & 0 & 0 & \cdots & 0
\end{array}\right)
\end{gathered}
$$

In the matrix $B$, the $E_{k}$ is a $(d+N) \times \mathfrak{X}^{(k)}$-matrix for $0 \leq k \leq N$, defined by, for $k>0$,

$$
E_{k}\left(i, x^{(k)}\right)=\left\{\begin{array}{ll}
0 & \text { for } 1 \leq i \leq d+k-1 \\
T^{k-1, k}\left(x_{d+k}^{(k-1)}, x^{(k)}\right)=\varphi_{d+k}\left(x_{d+k}^{(k-1)}, x^{(k)}\right) & \text { for } i=d+k \\
0 & d+k+1 \leq i \leq d+N
\end{array},\right.
$$


and, for $k=0$,

$$
E_{0}=\left(\begin{array}{c}
\Phi^{d} \\
0 \\
\vdots \\
0
\end{array}\right) \cdot \begin{aligned}
& \uparrow d \\
&
\end{aligned}
$$

Defining $D:=\mathbb{1}_{\mathfrak{Y}}+D_{0}$, the point measure on $\mathfrak{Y}$ is determinantal with correlation kernel, given by

$$
\begin{aligned}
\widetilde{\mathbb{K}}:=\mathbb{1}_{\mathfrak{Y}}-\left.\left(\mathbb{1}_{\mathfrak{Y}}+L_{\mathfrak{Y} \otimes} \otimes \mathfrak{Y}\right)^{-1}\right|_{\mathfrak{Y} \times \mathfrak{Y}} & =\mathbb{1}_{\mathfrak{Y}}-\left(\begin{array}{cc}
A & B \\
C & D
\end{array}\right)^{-1} \\
& =\mathbb{1}_{\mathfrak{Y}}-\left.\left(\begin{array}{cc}
\star & \star \\
\star & D^{-1}-D^{-1} C M^{-1} B D^{-1}
\end{array}\right)\right|_{\mathfrak{Y} \times \mathfrak{Y}} \\
& =\mathbb{1}_{\mathfrak{Y}}-D^{-1}+D^{-1} C M^{-1} B D^{-1},
\end{aligned}
$$

with $M=B D^{-1} C-A$; it will be shown that this is precisely the matrix $M$ in 62). To do so, we need to compute the different matrices appearing in the expression 68. It is easily seen that

$$
D^{-1}=\left(\begin{array}{cccc}
\mathbb{1} & W_{[0,1)} & \cdots & W_{[0, N)} \\
& & & \vdots \\
0 & \mathbb{1} & & \\
\vdots & \ddots & & W_{[N-1, N)} \\
0 & 0 & 0 & \mathbb{1}
\end{array}\right) \text { with } W_{[n, m)}:=W_{n, n+1} \ldots W_{m-1, m} \mathbb{1}_{m>n} .
$$

Coordinatewise, the $\mathfrak{X}^{(n)} \times \mathfrak{X}^{(m)}$-matrices $W_{[n, m)}$ are given by

$$
\begin{aligned}
W_{[n, m)}\left(x^{(n)}, x^{(m)}\right) & =\varphi_{d+1+n}\left(x^{(n)}, x^{(n+1)}\right) * \ldots * \varphi_{d+m}\left(x^{(m-1)}, x^{(m)}\right) \\
& =\varphi^{d+1+n, d+1+m}\left(x^{(n)}, x^{(m)}\right) .
\end{aligned}
$$

Moreover,

$B D^{-1}=\left(\stackrel{\stackrel{x^{(0)}}{\leftrightarrow}}{E_{0}}, \quad E_{0} W_{[0,1)} \stackrel{\stackrel{x^{(1)}}{\rightleftarrows}}{+} E_{1}, \ldots, \sum_{k=0}^{m-1} E_{k} W_{[k, m)} \stackrel{\stackrel{x_{(m)}}{\rightleftarrows}}{\longrightarrow} E_{m}, \ldots, \sum_{k=0}^{N-1} E_{k} W_{[k, N)} \stackrel{\stackrel{x^{(N)}}{\rightleftarrows}}{+} E_{N}\right) \downarrow d+N$, 
where

$$
\begin{aligned}
E_{k} W_{[k, m)}= & \left(\begin{array}{c}
O \\
\varphi_{d+k}\left(x_{d+k}^{(k-1)}, x^{(k)}\right) \\
O
\end{array}\right) \leftarrow d+k \\
& \times\left(\varphi^{d+1+k, d+1+m}\left(x^{(k)}, x_{j}^{(m)}\right) \delta_{m>k}\right) \\
= & \left(\begin{array}{c}
O \\
\left(\varphi_{d+k} * \varphi^{d+1+k, d+1+m}\right)\left(x_{d+k}^{(k-1)}, x^{(m)}\right) \delta_{m>k}
\end{array}\right) \leftarrow d+k .
\end{aligned}
$$

Hence $B D^{-1}$ consists of $N+1$ column-blocks, the $m$ th block being given below, for $0 \leq m \leq N$, with $O_{N-m}$ a $(N-m) \times \mathfrak{X}^{(m)}$ matrix of zeros,

$$
\begin{aligned}
& \sum_{k=0}^{m-1} E_{k} W_{[k, m)}+E_{m}=
\end{aligned}
$$

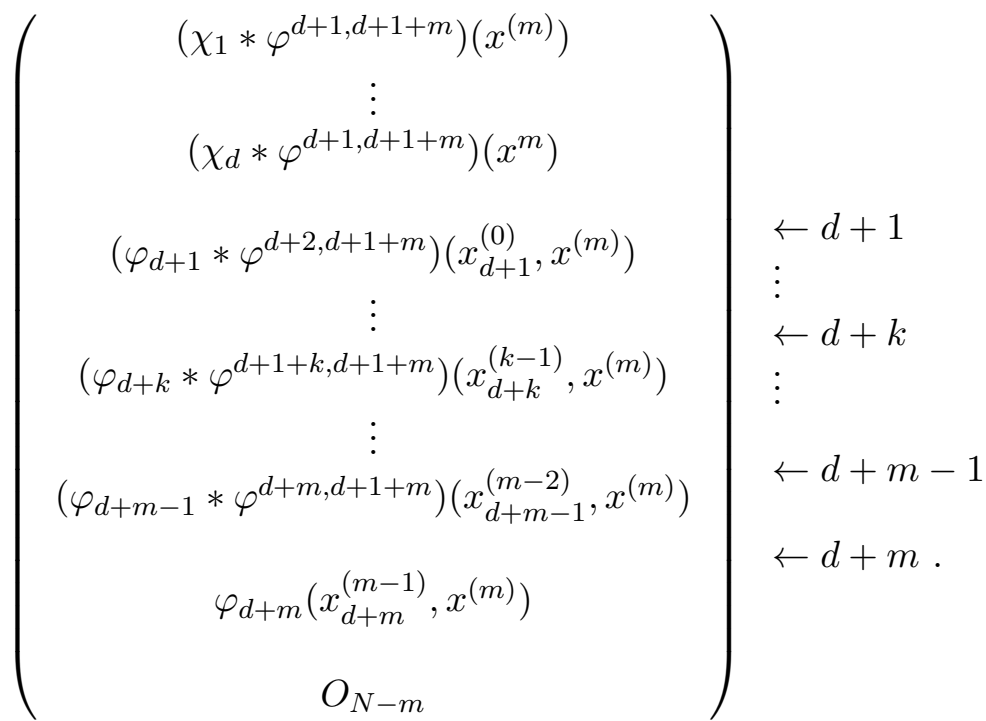

Notice that in the matrix above, we have that the arguments $x_{d+k}^{(k-1)}=$ virt. Moreover

$$
D^{-1} C=\left(\begin{array}{c}
W_{[0, N)} \Psi^{d+N} \\
\vdots \\
W_{[k, N)} \Psi^{d+N} \\
\vdots \\
W_{[N-1, N)} \Psi^{d+N} \\
\Psi^{d+N}
\end{array}\right)=\left(\begin{array}{l}
\psi_{j}^{(1)}\left(x^{(0)}\right) \\
\vdots \\
\psi_{j}^{(k+1)}\left(x^{(k)}\right) \\
\vdots \\
\psi_{j}^{(N)}\left(x^{(N-1)}\right) \\
\psi_{j}^{(N+1)}\left(x^{(N)}\right)
\end{array}\right)_{1 \leq j \leq d+N}, \text { with } \psi_{j}^{(N+1)}=\psi_{j}
$$


because, for $0 \leq k \leq N-1$ and $1 \leq j \leq d+N$, we have, using the notation of 60 ,

$$
\begin{aligned}
\left(W_{[k, N)} \Psi^{d+N}\right)\left(x^{(k)}, j\right) & =W_{[k, N)}\left(x^{(k)}, x^{(N)}\right) \Psi^{d+N}\left(x^{(N)}, j\right) \\
& =\varphi^{d+1+k, d+1+N}\left(x^{(k)}, \circ\right) * \psi_{j}(\circ)=\psi_{j}^{(k+1)}\left(x^{(k)}\right),
\end{aligned}
$$

and $\Psi^{d+N}\left(x^{(N)}, j\right)=\psi_{j}^{(N+1)}\left(x^{(N)}\right)=\psi_{j}\left(x^{(N)}\right)$. Hence the $(m+1, n+1)^{t h}$ block of $\left(D^{-1} C\right) M^{-1}\left(B D^{-1}\right)$ reads for ${ }^{10} 0 \leq m, n \leq N$,

$$
\begin{aligned}
& \sum_{\substack{1 \leq k \leq d+N \\
1 \leq \ell \leq d}} \psi_{k}^{(m+1)}\left(x^{(m)}\right)\left(M^{-1}\right)_{k \ell}\left(\chi_{\ell} * \varphi^{d+1, d+1+n}\right)\left(x^{(n)}\right) \\
+ & \sum_{\substack{1 \leq k \leq d+N \\
d+1 \leq \ell \leq d+n}} \psi_{k}^{(m+1)}\left(x^{(m)}\right)\left(M^{-1}\right)_{k \ell}\left(\varphi_{\ell} * \varphi^{\ell+1, d+1+n}\right)\left(x_{\ell}^{(\ell-d-1)}, x^{(n)}\right),
\end{aligned}
$$

with $x_{\ell}^{(\ell-d-1)}=$ virt and the $(m+1, n+1)^{t h}$ block of $\left(\mathbb{1}_{\mathfrak{Y}}-D^{-1}\right)$ reads

$$
-W_{m, n}=\varphi^{d+1+m, d+1+n}\left(x^{(m)}, x^{(n)}\right) .
$$

Then upon setting $x=x^{(m)}$ and $y=x^{(n)}$ as running variables, and combining the two last equations, we conclude that for $0 \leq m, n \leq N$,

$$
\tilde{\mathbb{K}}(m, x ; n, y)=\left(\mathbb{1}_{\mathfrak{Y}}-D^{-1}+D^{-1} C M^{-1} B D^{-1}\right)_{m+1, n+1}
$$

gives indeed formula (61), using the notation 64 for $\varphi^{n, m}$. Finally, we have $M=\left(M_{i j}\right)_{1 \leq i, j \leq d+N}=B D^{-1} C=\left(B D^{-1}\right) C$, whose entries, upon using 69 , are given by

$$
M_{i, j}= \begin{cases}\left(\chi_{i} * \varphi^{d+1, d+1+N}\right)(\bullet, \circ) * \psi_{j}(\circ), & \text { for } 1 \leq i \leq d, \\ \left(\varphi_{i} * \varphi^{i+1, d+1+N}\right)(v i r t, \circ) * \psi_{j}(\circ), & \text { for } d+1 \leq i \leq d+N-1, \\ \varphi_{d+N}(\text { virt }, \circ) * \psi_{j}(\circ) & \text { for } i=d+N .\end{cases}
$$

This ends the proof of Proposition 4.2 .

Remark: For each each $0 \leq m \leq N$, consider the linear space $V_{m}$,

$$
V_{m}:=\operatorname{span}\{\text { functions in brackets of }(69)\}=\operatorname{span}\left\{\phi_{j}^{(m)}, 1 \leq j \leq d+m\right\},
$$

where $\phi_{j}^{(m)}, 1 \leq j \leq d+m$ is a new basis of $V_{m}$, defined by the conditions $\phi_{i}^{(m)} * \psi_{i}^{(m)}=\mathbb{1}_{i=j}$ for $1 \leq i, j \leq d+m$. Assuming this new basis is such that

\footnotetext{
${ }^{10}$ In the second summation below, we also have $\varphi^{d+1+n, d+1+n}=1$.
} 
$\varphi_{d+m}(\operatorname{virt}, x)=c_{m} \phi_{d+m}^{(m)}(x), c_{m} \neq 0,0 \leq m \leq N$ (Assumption A in [9, 7]), then it is shown that the kernel (68) has the following simple form:

$$
\mathbb{K}_{q}(m, x ; n, y)=-\varphi^{d+1+m, d+1+n}(x, y)+\sum_{1}^{d+n} \psi_{j}^{(m)}(x) \phi_{j}^{(n)}(y) .
$$

Assumption A can in our case indeed be achieved for some appropriate choice of basis, which at the end of the day leads to a representation of the kernel in terms of $(2,3)$-mops on the circle.

\section{Transforming $M$ into $\tilde{M}$ and computing its inverse}

The purpose of this section is to transform the matrix $M$, which appears in the kernel 61) for the multi-cut case, into a new matrix $\tilde{M}$, which is easily invertible. To do so, one needs to define $D_{n}:=\operatorname{diag}\left(e_{n-1}, \ldots, e_{0}\right), \Delta_{n}^{(q)}$ a Vandermonde and $\Pi_{n}$ a permutation matrix, $\left(\right.$ recall $\left.\mathcal{P}_{N-1}(z)=\sum_{i=1}^{N} e_{i-1} z^{i}\right)$

$\Delta_{n}^{(q)}:=\left(\begin{array}{cccc}1 & 1 & \ldots & 1 \\ \left(q^{-1}\right)^{n-1} & \left(q^{-1}\right)^{n-2} & \ldots & 1 \\ \left(q^{-2}\right)^{n-1} & \left(q^{-2}\right)^{n-2} & \ldots & 1 \\ \vdots & \vdots & & \vdots \\ \left(q^{-(n-1)}\right)^{n-1} & \left(q^{-(n-1)}\right)^{n-2} & \ldots & 1\end{array}\right), \Pi_{n}:=\left(\begin{array}{cccc}O & & & \\ & & 1 & \\ & & 1 & \\ & 1 & & O \\ 1 & & & \end{array}\right)$.

Proposition 5.1 The inverse of the matrix $\widetilde{M}:=M T^{\top-1}$ reads as follows:

$$
\widetilde{M}^{-1}=\left(\begin{array}{c|c}
\mathbb{1}_{d} & O \\
\hline O & \left(\delta_{2 d+N-i+1, j}(q ; q)_{i-d-1}\right)_{d+1 \leq i, j \leq d+N}
\end{array}\right)
$$

where $T$ is a $x$ and $y$ independent square matrix of size $d+N$,

$$
T=\left(\begin{array}{c|c}
\mathbb{1}_{d} & O \\
\hline O & T_{N}
\end{array}\right)
$$

with

$$
T_{N}:=\operatorname{diag}\left(q^{(N-j) d}\right)_{1 \leq j \leq N} \Delta_{N}^{(q)-1} \Pi_{N} D_{N}^{-1} \operatorname{diag}\left(q^{-(d+N)(d+N-j)}\right)_{1 \leq j \leq N} .
$$

Before giving the proof of Proposition 5.1, we need Lemma 5.2 below. It is useful to modify the matrix $H$ of size $d+N$ (as in (37) ) to $\widetilde{H}$, by an $x$ and $y$ independent transformation $T$ of block form, so as to be close to the Schur case rather than the skew-Schur case; that is, so that the $(d+N, N)$-block of the matrix $\widetilde{H}$ are pure powers of $q$. 
Lemma 5.2 Multiplying the block matrix $H$ with the matrix $T$ above (72), yields $\left(O_{n, m}\right.$ denotes a $n \times m$-matrix of zeros $)$ :

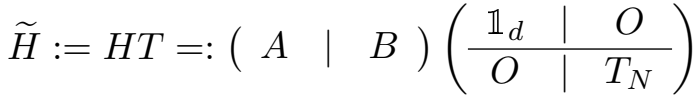

$$
\begin{aligned}
& =\left(\left[h_{x_{i}-y_{j}}\left(q^{d}, \ldots, q^{d+N-1}\right)\right]_{\substack{1 \leq i \leq d+N \\
1 \leq j \leq d}} \mid\left[q^{x_{i}(d+N-j)}\right]_{\substack{1 \leq i \leq d+N \\
1 \leq j \leq N}}\right) \\
& =\left(\begin{array}{c|c}
{\left[q^{d\left(x_{i}-y_{j}\right)} \mathcal{P}_{N-1}\left(q^{x_{i}-y_{j}}\right)\right]_{\substack{1 \leq i \leq c \\
1 \leq j \leq d}}} & {\left[q^{d x_{i}} q^{x_{i}(N-j)}\right]_{\substack{1 \leq i \leq d+N \\
1 \leq j \leq N}}} \\
O_{d+b, d} & \substack{1 \leq j \leq d\\
}
\end{array}\right.
\end{aligned}
$$

Proof: At first, we have the following identity (Remember: $\mathcal{P}_{n-1}(z)=$ $\sum_{i=1}^{n} e_{i-1} z^{i-1} ;$ see formula 34 )

$$
\left(z^{n-1}, \ldots, z^{0}\right)=\left(\mathcal{P}_{n-1}\left(z\left(q^{-1}\right)^{n-1}\right), \ldots, \mathcal{P}_{n-1}(z)\right) \widetilde{T}, \quad \text { with } \quad \widetilde{T}_{n}=\Delta_{n}^{(q)^{-1}} \Pi_{n} D_{n}^{-1} .
$$

Indeed, $\widetilde{T}$ is such that:

$$
\begin{aligned}
z^{\ell-1} & =\sum_{k=1}^{n} \mathcal{P}_{n-1}\left(z\left(q^{-1}\right)^{n-k}\right) \widetilde{T}_{k, n-\ell+1} \\
& =\sum_{i=1}^{n} z^{i-1} e_{i-1} \sum_{k=1}^{n}\left(q^{-(i-1)}\right)^{(n-k)} \widetilde{T}_{k, n-\ell+1},
\end{aligned}
$$

implying, for all $1 \leq \ell, i \leq n$, that $e_{i-1}^{-1} \delta_{i, \ell}=\sum_{k=1}^{n}\left(q^{-(i-1)}\right)^{(n-k)} \widetilde{T}_{k, n-\ell+1}$, which is statement $(74)$ in matrix notation.

Apply this to the matrix $H$ and $n=N$,

$$
H=(\overbrace{A}^{d} \mid \overbrace{B}^{N}),
$$

where the matrix $A$ of size $(d+N, d)$ consists of the $d$ columns corresponding to the points in the lower-cut $y_{1}, \ldots, y_{d}$ and the matrix $B$ of size $(d+N, N)$ to the points $y_{d+1}, \ldots, y_{d+N}$ to the left of $\mathbf{P} \cap\{\mathbf{y}=\mathbf{0}\}$. One now uses formula (35) and notices that $N-1+x_{i}-y_{d+j} \geq 0$ for $i, j \geq 1$; this enables us to omit the indicator in the formula for the matrix $B=\left(B_{i j}\right)_{\substack{1 \leq i \leq d+N \\ 1 \leq j \leq N}}$ :

$$
\begin{aligned}
B_{i j} & =\left(h_{x_{i}-y_{d+j}}\left(q^{d}, \ldots, q^{d+N-1}\right)\right. \\
& =q^{d\left(x_{i}+d+j\right)} \mathcal{P}_{N-1}\left(q^{x_{i}+d+j}\right) \mathbb{1}_{N-1+x_{i}-y_{d+j} \geq 0} \\
& =\left.q^{d\left(x_{i}+d+N\right)} \mathcal{P}_{N-1}\left(z\left(q^{-1}\right)^{N-j}\right)\right|_{z=q^{x_{i}+d+N}}\left(q^{-1}\right)^{(N-j) d},
\end{aligned}
$$


and thus, in matrix notation,

$$
\operatorname{diag}\left(q^{-d\left(x_{i}+d+N\right)}\right) B \operatorname{diag}\left(q^{(N-j) d}\right)=\left.\left(\left.\mathcal{P}_{N-1}\left(z\left(q^{-1}\right)^{N-j}\right)\right|_{z=q^{x_{i}+d+N}}\right)\right|_{\substack{1 \leq i \leq d+N \\ 1 \leq j \leq N}}
$$

From 74 , it follows that for $\widetilde{T}_{N}=T$, as in 72 ,

$$
\operatorname{diag}\left(q^{-d\left(x_{i}+d+N\right)}\right) B \operatorname{diag}\left(q^{(N-j) d}\right) \widetilde{T}_{N}=\left(\left.\left(z^{N-j}\right)\right|_{z=q^{x_{i}+d+N}}\right) \underbrace{}_{\substack{1 \leq i \leq d+N \\ 1 \leq j \leq N}}
$$

and so

$$
\begin{aligned}
B \operatorname{diag}\left(q^{(N-j) d}\right) \widetilde{T}_{N} & =\left(q^{\left(d+N+x_{i}\right)(d+N-j)}\right)_{\substack{1 \leq i \leq d+N \\
1 \leq j \leq N}} \\
& =\left(q^{x_{i}(d+N-j)}\right)_{\substack{1 \leq i \leq d+N \\
1 \leq j \leq N}} \cdot \operatorname{diag}\left(q^{(d+N)(d+N-j)}\right)_{1 \leq j \leq N},
\end{aligned}
$$

which we rewrite as

$$
B T_{N}=\left(q^{x_{i}(d+N-j)}\right) \underset{\substack{1 \leq i \leq d+N \\ 1 \leq j \leq N}}{\substack{1 \\ \text { ind }}}
$$

for $T_{N}$ given in terms of $\widetilde{T}_{N}$, as defined in 74 ,

$$
T_{N}:=\operatorname{diag}\left(q^{(N-j) d}\right) \widetilde{T}_{N} \operatorname{diag}\left(q^{-(d+N)(d+N-j)}\right)_{1 \leq j \leq N}
$$

This corresponds to the matrix $T_{N}$ in 72 . The multiplication by $T$ leaves the first $d$ columns unchanged. Also the last equality in 73 is an immediate consequence of formula (35) which includes the indicator function, since by hypothesis $N-$ $1+x_{c}-y_{1} \geq 0$. This ends the proof of Lemma 5.2 .

Proof of Proposition 5.1. Inserting (60) in the matrix $M$, as defined in (62), one sees that $M$ can be written, for appropriate choices of $\Phi_{i}$ 's, as:

$$
M_{i j}=\Phi_{i}(\cdot, \circ) * \psi_{j}(\circ)=\Phi_{i}(\cdot, \circ) * \sum_{\alpha=1}^{d+N} H_{j \alpha}^{-1} \mathbb{1}_{z=x_{\alpha}}(\circ), \quad 1 \leq i, j \leq N+d .
$$

Since $\widetilde{H}=H T$ in Lemma 5.2 , this suggests transforming the $\psi_{k}$ into a new expression $\tilde{\psi}_{\alpha}$, defined by

$$
\widetilde{\psi}_{j}=\sum_{\alpha=1}^{d+N} T_{j \alpha}^{-1} \psi_{\alpha}=\sum_{\alpha=1}^{d+N} \widetilde{H}_{j \alpha}^{-1} \mathbb{1}_{z=x_{\alpha}}
$$


and consequently $\psi_{k}^{(m+1)}$ into a new $\widetilde{\psi}_{k}^{(m+1)}$ as defined in 60 and 65 ,

$$
\widetilde{\psi}_{k}^{(m+1)}(x):=\sum_{\ell=1}^{d+N}\left(\widetilde{H}^{-1}\right)_{k \ell} h_{x_{\ell}-x}\left(q^{d+m}, \ldots, q^{d+N-1}\right) .
$$

From (61), 62 and (60), this in turn induces a transformation on $M$, preserving the kernel (61):

$$
M_{i j}=\left(\Phi_{i} * \sum_{\alpha} T_{j \alpha} \widetilde{\psi}_{\alpha}\right)=\sum_{\alpha} T_{j \alpha}\left(\Phi_{i} * \widetilde{\psi}_{\alpha}\right)=\sum_{\alpha}\left(\Phi_{i} * \widetilde{\psi}_{\alpha}\right) T_{\alpha j}^{\top}=\left(\widetilde{M} T^{\top}\right)_{i j},
$$

where $\widetilde{M}=M T^{\top-1}$ is the matrix $M$ as in 75 , but with $\psi_{\alpha}$ replaced by $\tilde{\psi}_{\alpha}$. In view of the form of the kernel (61), we check, using the previous expression, that the kernel is indeed preserved. It suffices by (61) and (60) to check the identity

$$
\begin{aligned}
\sum_{k} \psi_{k} M_{k \ell}^{-1} & =\sum_{k} \sum_{j} H_{k j}^{-1} \mathbb{1}_{z=x_{j}} \sum_{\alpha} T_{k \alpha}^{\top-1} \widetilde{M}_{\alpha, \ell}^{-1} \\
& =\sum_{\alpha, j} \mathbb{1}_{z=x_{j}} \widetilde{M}_{\alpha, \ell}^{-1} \sum_{k} H_{k j}^{-1} T_{k \alpha}^{\top-1} \\
& =\sum_{\alpha, j}(H T)_{\alpha, j}^{-1} \mathbb{1}_{z=x_{j}} \widetilde{M}_{\alpha, \ell}^{-1} \\
& =\sum_{\alpha, j}(\tilde{H})_{\alpha, j}^{-1} \mathbb{1}_{z=x_{j}} \widetilde{M}_{\alpha \ell}^{-1}=\sum_{\alpha} \tilde{\psi}_{\alpha} \widetilde{M}_{\alpha \ell}^{-1},
\end{aligned}
$$

inducing a similar relation $\sum_{k} \psi_{k}^{(m+1)} M_{k \ell}^{-1}=\sum_{\alpha} \tilde{\psi}_{\alpha}^{(m+1)} \widetilde{M}_{\alpha \ell}^{-1}$.

We now compute explicitly $\widetilde{M}$ :

For $1 \leq i \leq d$ and $1 \leq j \leq d+N$, setting $n=d$ and $m=d+N$ in (64)

$$
\begin{aligned}
M_{i j}=\chi_{i} * \varphi_{d+1} * \ldots * \varphi_{d+N} * \psi_{j} & =\left(\varphi_{d+1} \ldots \varphi_{d+N}\right)\left(y_{i}, \cdot\right) * \psi_{j}(\cdot) \\
& =\sum_{k=1}^{N+d}\left(H^{-1}\right)_{j, k} h_{x_{k}-y_{i}}\left(q^{d}, \ldots, q^{N+d-1}\right) \\
& =\sum_{k=1}^{N+d}\left(H^{-1}\right)_{j, k} H_{k, i}=\mathbb{I}_{i j} \\
\widetilde{M}_{i j}=\left(M T^{\top-1}\right)_{i j}=\mathbb{I}_{i j}, &
\end{aligned}
$$

since the left $(d+N) \times d$-block of $T$ is $\left(\begin{array}{c}\mathbb{1}_{d} \\ O\end{array}\right)$. From this discussion it follows that the $\widetilde{\psi}_{j}, \widetilde{H}, \widetilde{M}$ are related to each other in the same way as the $\psi_{j}, H, M$, via the transformation $\psi_{j} \mapsto \widetilde{\psi}_{j}, H \mapsto \widetilde{H}, M \mapsto \widetilde{M}$. 
For $d+1 \leq i \leq d+N$ and $1 \leq j \leq d+N$, one has, using the same identity for $\varphi_{i}($ virt,$\cdot) *\left(\varphi_{i+1} * \ldots * \varphi_{d+N}\right)(\cdot, \circ) * \psi_{j}(\circ)$ as in 64 , but for $\psi_{j}$ replaced by $\widetilde{\psi}_{j}$, one checks that

$$
\begin{aligned}
\widetilde{M}_{i j} & =\varphi_{i}(\text { virt }, \cdot) *\left(\varphi_{i+1} * \ldots * \varphi_{d+N}\right)(\cdot, \circ) * \widetilde{\psi}_{j}(\circ) \\
& =\frac{1}{(1-q) \ldots\left(1-q^{N-i+d}\right)} \sum_{\ell=1}^{N+d}\left(\widetilde{H}^{-1}\right)_{j \ell} q^{(i-1) x_{\ell}} \\
& =\frac{1}{(1-q) \ldots\left(1-q^{N-i+d}\right)} \sum_{\ell=1}^{N+d}\left(\widetilde{H}^{-1}\right)_{j \ell} \widetilde{H}_{\ell, 2 d+N-i+1} \\
& =\frac{1}{(1-q) \ldots\left(1-q^{N-i+d}\right)} \delta_{j, 2 d+N-i+1}=\frac{\delta_{j, 2 d+N-i+1}}{(q ; q)_{d+N-i}} .
\end{aligned}
$$

So, the matrix $\widetilde{M}$ reads:

$$
\widetilde{M}=\left(\begin{array}{c|c}
\mathbb{1}_{d} & O \\
\hline O & \left(\frac{\delta_{2 d+N-i+1, j}}{(q ; q)_{d+N-i}}\right)_{d+1 \leq i, j \leq d+N}
\end{array}\right)
$$

and so

$$
\widetilde{M}^{-1}=\left(\begin{array}{c|ccc}
\mathbb{1}_{d} & \multicolumn{3}{|c}{O} \\
\hline & & & \\
& & & \\
O & O & \cdot & \\
& & . & \\
& & & \\
& (q ; q)_{N-1} & &
\end{array}\right),
$$

establishing Proposition 5.1.

\section{The integral representation of the $\mathbb{K}_{q}$-kernel}

The kernel will be given in terms of multiple contour integrals, one of them being a $d+2$-fold integral. But at first, we express the kernel in terms of the basic functions $h_{r}$ and $\widetilde{\psi}_{k}^{(m+1)}(x)$, as in 76 . 
Proposition 6.1 For $0 \leq m, n \leq N$, the q-kernel reads as follows:

$$
\begin{aligned}
& \mathbb{K}_{q}(m, x ; n, y) \\
& =-h_{y-x}\left(q^{d+m}, \ldots, q^{d+n-1}\right) \mathbb{1}_{n>m} \\
& \quad+q^{d y} \sum_{k=1}^{n} \widetilde{\psi}_{d+N-k+1}^{(m+1)}(x) q^{(k-1) y} \prod_{i=n+1}^{N}\left(1-q^{i-k}\right) \\
& \quad+\sum_{k=1}^{d} \widetilde{\psi}_{k}^{(m+1)}(x) h_{y-y_{k}}\left(q^{d}, \ldots, q^{d+n-1}\right) .
\end{aligned}
$$

Proof: We use the kernel in Proposition 4.2. Equality $\stackrel{*}{=}$ follows from the inverse (77) of the matrix $\widetilde{M}$. Using Lemma 4.3, we then have:

$$
\begin{aligned}
& \mathbb{K}_{q}(m, x ; n, y) \\
= & -\varphi_{d+m+1} * \ldots * \varphi_{d+n}(x, y) \\
& +\sum_{\substack{1 \leq k \leq d+N \\
1 \leq \ell \leq d}} \widetilde{\psi}_{k}^{(m+1)}(x)\left(\widetilde{M}^{-1}\right)_{k \ell}\left(\chi_{\ell} * \varphi_{d+1} * \ldots * \varphi_{d+n}\right)(y) \\
& +\sum_{\substack{1 \leq k \leq d+N \\
d+1 \leq \ell \leq d+n}} \widetilde{\psi}_{k}^{(m+1)}(x)\left(\widetilde{M}^{-1}\right)_{k \ell}\left(\varphi_{\ell}(\operatorname{virt}, \cdot) *\left(\varphi_{\ell+1} * \cdots * \varphi_{d+n}\right)(\cdot, y)\right) \\
* & -\varphi_{d+m+1} * \ldots * \varphi_{d+n}(x, y) \\
& +\sum_{1 \leq k \leq d} \widetilde{\psi}_{k}^{(m+1)}(x)\left(\chi_{k} * \varphi_{d+1} * \ldots * \varphi_{d+n}\right)(y) \\
& +\sum_{\substack{d+1 \leq k \leq d+N \\
d+1 \leq \ell \leq d+n}} \widetilde{\psi}_{k}^{(m+1)}(x) \delta_{2 d+N-k+1, \ell}(q ; q)_{k-d-1}\left(\varphi_{\ell}(\operatorname{virt}, \cdot) *\left(\varphi_{\ell+1} * \cdots * \varphi_{d+n}\right)(\cdot, y)\right) \\
& =-h_{y-x}\left(q^{d+m}, \ldots, q^{d+n-1}\right) \mathbb{1}_{n>m}+\sum_{1 \leq k \leq d} \widetilde{\psi}_{k}^{(m+1)}(x) h_{y-y_{k}}\left(q^{d}, \ldots, q^{d+n-1}\right) \\
& +\sum_{d+N-(n-1) \leq k \leq d+N} \widetilde{\psi}_{k}^{(m+1)}(x) q^{(2 d+N-k) y} \frac{(q ; q)_{k-d-1}}{(q ; q)_{k-d-(N-n+1)}} .
\end{aligned}
$$


Then relabeling indices, we find:

$$
\begin{aligned}
\mathbb{K}_{q}(m, x ; n, y) \\
=-h_{y-x}\left(q^{d+m}, \ldots, q^{d+n-1}\right) \mathbb{1}_{n>m}+\sum_{1 \leq k \leq d} \widetilde{\psi}_{k}^{(m+1)}(x) h_{y-y_{k}}\left(q^{d}, \ldots, q^{d+n-1}\right) \\
\quad+\sum_{0 \leq k \leq n-1} \widetilde{\psi}_{d+N-k}^{(m+1)}(x) q^{(d+k) y} \frac{(q ; q)_{N-k-1}}{(q ; q)_{n-k-1}} \\
=-h_{y-x}\left(q^{d+m}, \ldots, q^{d+n-1}\right) \mathbb{1}_{n>m} \\
\quad+q^{d y} \sum_{k=1}^{n} \widetilde{\psi}_{d+N-k+1}^{(m+1)}(x) q^{(k-1) y} \prod_{n+1}^{N}\left(1-q^{i-k}\right) \\
\quad+\sum_{k=1}^{d} \widetilde{\psi}_{k}^{(m+1)}(x) h_{y-y_{k}}\left(q^{d}, \ldots, q^{d+n-1}\right),
\end{aligned}
$$

establishing (78).

Theorem 6.2 below gives an integral representation of the kernel for the $\mathbb{K}_{q^{-}}$ process. To do so, recall from (34), (35), the notation $q_{i}=q^{x_{i}}$ and the identities and definitions:

$$
h_{r}\left(q^{d}, \ldots, q^{d+n}\right)=q^{r d} \mathcal{P}_{n}\left(q^{r}\right) \mathbb{1}_{n+r \geq 0} \quad \text { and } \quad \tilde{\mathcal{P}}_{n-1}^{y}(z):=q^{-d y} \mathcal{P}_{n-1}\left(z q^{-y}\right) .
$$

and also that for $1 \leq r \leq d, 0 \leq m \leq N-1$ and $n \geq 1$,

$$
\begin{aligned}
h_{x_{k}-x}\left(q^{d+m}, \ldots, q^{d+N-1}\right) & =q^{\left(x_{k}-x\right)(d+m)} \mathcal{P}_{N-m-1}\left(q^{x_{k}-x}\right) \mathbb{1}_{x_{k} \geq x} \\
h_{y-y_{r}}(\bullet)=h_{y-y_{r}}\left(q^{d}, \ldots, q^{d+n-1}\right) & =\frac{1}{2 \pi \mathrm{i}} \oint_{\Gamma_{0}} \frac{d u}{u^{y-y_{r}+1}} \prod_{\ell=1}^{n} \frac{1}{1-u q^{d-1+\ell}} .
\end{aligned}
$$

The reader is also reminded of the expression, considered in Lemma 2.1.

$$
\Phi_{q}\left(z^{-1}\right):=\left(q^{N-1} ; q^{-1}\right)_{N-n 2} \phi_{1}\left(q^{-1}, q^{n-1} ; q^{N-1} \mid q^{-1}, z^{-1}\right) .
$$

In the next Theorem, one will need $q$-analogues of the three determinants (51), depending on the points $\mathbf{y}_{\text {cut }}=\left(y_{1}, \ldots, y_{d}\right)$ in the lower-cuts. The second will involve the $q$-analogue $h_{y-y_{r}}(\bullet):=h_{y-y_{r}}\left(q^{d}, \ldots, q^{d+n-1}\right)$ of the function $h_{y-y_{r}}\left(1^{n}\right)$ which has the integral representation 80 . The definitions are:

$$
\Delta_{q, d}^{\left(\mathbf{y}_{\text {cut }}\right)}\left(u_{1}, \ldots, u_{d}\right):=\operatorname{det}\left(\tilde{\mathcal{P}}_{N-1}^{y_{\beta}}\left(u_{\alpha}\right)\right)_{1 \leq \alpha, \beta \leq d}
$$




$$
\begin{aligned}
& \widetilde{\Delta}_{q, d}^{\left(\mathbf{y}_{\text {cut }}\right)}\left(w ; u_{2}, \ldots, u_{d}\right)=\operatorname{det}\left(\begin{array}{ccc}
w^{y_{1}} & \ldots & w^{y_{d}} \\
\tilde{\mathcal{P}}_{N-1}^{y_{1}}\left(u_{2}\right) & \ldots & \tilde{\mathcal{P}}_{N-1}^{y_{d}}\left(u_{2}\right) \\
\vdots & & \vdots \\
\tilde{\mathcal{P}}_{N-1}^{y_{1}}\left(u_{d}\right) & \ldots & \tilde{\mathcal{P}}_{N-1}^{y_{d}}\left(u_{d}\right)
\end{array}\right) \\
& \widetilde{\Delta}_{q, d, n}^{\left(\mathbf{y}_{\text {cut }}\right)}\left(y ; u_{2}, \ldots, u_{d}\right):=\oint_{\Gamma_{0}} \frac{d w}{2 \pi \mathrm{i} w^{y+1} \prod_{k=1}^{n}\left(1-w q^{d+k-1}\right)} \widetilde{\Delta}_{q, d}^{\left(\mathbf{y}_{\text {cut }}\right)}\left(w, u_{2}, \ldots, u_{d}\right) \\
& =\operatorname{det}\left(\begin{array}{ccc}
h_{y-y_{1}}(\bullet) & \ldots & h_{y-y_{d}}(\bullet) \\
\tilde{\mathcal{P}}_{N-1}^{y_{1}}\left(u_{2}\right) & \ldots & \tilde{\mathcal{P}}_{N-1}^{y_{d}}\left(u_{2}\right) \\
\vdots & & \vdots \\
\tilde{\mathcal{P}}_{N-1}^{y_{1}}\left(u_{d}\right) & \ldots & \tilde{\mathcal{P}}_{N-1}^{y_{d}}\left(u_{d}\right)
\end{array}\right) \text {. }
\end{aligned}
$$

The last expression is obtained by performing the $w$-integration on the first row of the matrix in $\widetilde{\Delta}_{q, d}^{\left(\mathbf{y}_{\text {cut }}\right)}$. The expression $\widetilde{\Delta}_{q, d, n}^{\left(\mathbf{y}_{\text {cut }}\right)}\left(y ; u_{2}, \ldots, u_{d}\right)$ clearly vanishes, when $y \leq y_{d}-1$, or what is the same, when $y$ is strictly to the left of the lower-cuts.

Theorem 6.2 For $0 \leq m, n \leq N$, the kernel for the $\mathbb{K}_{q}$-process reads as follows:

$$
\begin{aligned}
& q^{(d+m)(x-y)} \mathbb{K}_{q}(m, x ; n, y) \\
& =\left.\frac{(z q ; q)_{n-m-1}}{(q ; q)_{n-m-1}}\right|_{z=q^{y-x}} \mathbb{1}_{n>m} \mathbb{1}_{y \geq x} \\
& +\oint_{\Gamma\left(q^{x-y+\mathbb{N}}\right)} \frac{v^{m} d v}{2 \pi \mathrm{i}} \oint_{\Gamma_{\infty}} \frac{\Phi_{q}\left(z^{-1}\right) d z}{2 \pi \mathrm{i} z} \frac{\mathcal{P}_{N-m-1}\left(v q^{y-x}\right)}{z-v} \frac{Q_{q}\left(z q^{y}\right)}{Q_{q}\left(v q^{y}\right)} \frac{\Omega_{q}\left(v q^{y}, z q^{y}\right)}{\Omega_{q}(0,0)} \\
& +\frac{d}{q^{(d-1) y}} \oint_{\Gamma_{\Gamma\left(q^{x-y+\mathbb{N}}\right)}} \frac{v^{m} d v}{2 \pi i} \frac{\mathcal{P}_{N-m-1}\left(v q^{y-x}\right)}{Q_{q}\left(v q^{y}\right)} \frac{\widetilde{\Omega}_{q}\left(v q^{y}, y\right)}{\Omega_{q}(0,0)} \\
& =:\left(\mathbb{K}_{q}^{(0)}+\mathbb{K}_{q}^{(1)}+\mathbb{K}_{q}^{(2)}\right)(m, x ; n, y),
\end{aligned}
$$

where 11

$$
\begin{aligned}
\Omega_{q}(v, z):=\left(\prod_{j=1}^{d} \oint_{\Gamma\left(q_{1}, \ldots, q_{c}\right)}\right. & \left.\frac{d u_{j}}{2 \pi \mathrm{i} Q_{q}\left(u_{j}\right)}\right) \prod_{1}^{d} \frac{v-u_{i}}{z-u_{i}} \\
& \times \Delta_{d}\left(u_{1}, u_{2}, \ldots, u_{d}\right) \Delta_{q, d}^{\left(\mathbf{y}_{\text {cut }}\right)}\left(u_{1}, u_{2}, \ldots, u_{d}\right),
\end{aligned}
$$

and

$$
{ }^{11} \Omega_{q}=1 \text { for } d=0 . \quad F \text { and } \widetilde{\Omega}_{q}=1 \text { for } \mathrm{d}=1 .
$$




$$
\begin{aligned}
\widetilde{\Omega}_{q}(v, y) & :=\left(\prod_{j=2}^{d} \oint_{\Gamma\left(q_{1}, \ldots, q_{c}\right)} \frac{d u_{j}}{2 \pi \mathrm{i} Q_{q}\left(u_{j}\right)}\right) \\
& \times \Delta_{d}\left(v, u_{2}, \ldots, u_{d}\right) \widetilde{\Delta}_{q, d, n}^{\left(\mathbf{y}_{c u t}\right)}\left(q^{y}, u_{2}, \ldots, u_{d}\right) \\
& =\sum_{\substack{1 \leq r \leq d \\
y_{r} \leq y}}(-1)^{r-1} h_{y-y_{r}}(\bullet) F_{r}(v),
\end{aligned}
$$

with $\bullet=\left(q^{d}, \ldots, q^{d+n-1}\right)$ as in 80$)$ and

$$
F_{r}(v):=\left(\prod_{j=1}^{d-1} \oint_{\Gamma\left(q_{1}, \ldots, q_{c}\right)} \frac{d u_{j}}{2 \pi \mathrm{i} Q_{q}\left(u_{j}\right)}\right) \operatorname{det}\left(\widetilde{\mathcal{P}}_{N-1}^{y_{\beta}}\left(u_{\alpha}\right)\right) \substack{1 \leq \alpha \leq d-1 \\ 1 \leq \beta \leq d, \beta \neq r}_{\substack{1 \leq \alpha \\ \Delta^{\prime}}}\left(v, u_{1}, \ldots, u_{d-1}\right) .
$$

Finally, $\mathbb{K}_{q}^{(2)}(m, x ; n, y)=0$, when $y \leq y_{d}-1$; when $y$ is to the left of the lowercuts.

Proof of Theorem 6.2 Expressed as sum of residues, the multiple contour integral $\Omega_{q}(v, z), \widetilde{\Omega}_{q}(v, z)$ and $F_{r}(v)$ are as follows:

$$
\begin{aligned}
& \Omega_{q}(v, z):=\sum_{1 \leq i_{1}, \ldots, i_{d} \leq c} \frac{\Delta_{d}\left(q^{x_{i_{1}}}, \ldots, q^{x_{i_{d}}}\right) \Delta_{q, d}^{\left(\mathbf{y}_{\text {cut }}\right)}\left(q^{x_{i_{1}}}, \ldots, q^{x_{i_{d}}}\right)}{\prod_{\alpha=1}^{d} Q_{q}^{\prime}\left(q^{x_{i_{\alpha}}}\right)} \prod_{\alpha=1}^{d} \frac{v-q^{x_{i_{\alpha}}}}{z-q^{x_{i_{\alpha}}}} \\
& \widetilde{\Omega}_{q}(v, z):=\sum_{1 \leq i_{1}, \ldots, i_{d-1} \leq c} \frac{\Delta_{d}\left(v, q^{x_{i_{1}}}, \ldots, q^{x_{i_{d-1}}}\right) \widetilde{\Delta}_{q, d, n}^{\left(\mathbf{y}_{\text {cut }}\right)}\left(q^{y}, q^{x_{i_{1}}}, \ldots, q^{x_{i_{d}-1}}\right)}{\prod_{\alpha=1}^{d-1} Q_{q}^{\prime}\left(q^{x_{i_{\alpha}}}\right)}
\end{aligned}
$$

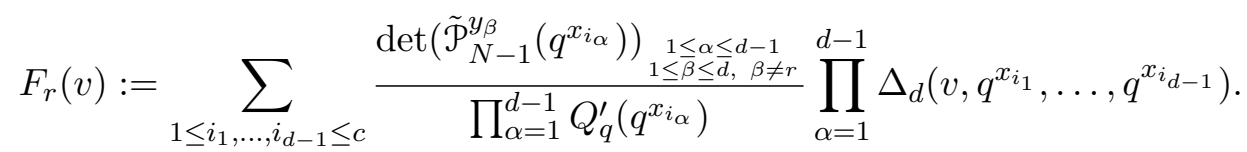

Expressing the determinant of $\widetilde{H}$ of size $(d+N)$ as a multiple contour integral, using the Laplace expansion for the determinant of a block matrix and using 73 for $\widetilde{H}$ and Lemma 3.4 in the third equality ${ }^{12}$, (remember $q_{i}:=q^{x_{i}}$ )

$$
\begin{aligned}
& \frac{\operatorname{det} \widetilde{H}}{\Delta_{d+N}\left(q_{1}, \ldots, q_{d+N}\right)} \\
= & \prod_{i=1}^{d+N} q^{d x_{i}} \frac{\operatorname{det}\left(\begin{array}{c}
\left(\tilde{\mathcal{P}}_{j-1}^{y_{j}}\left(q^{x_{i}}\right)\right)_{\substack{1 \leq i \leq c \\
1 \leq j \leq d}} \\
O_{d+b, d}
\end{array} \mid\left(q^{x_{i}(N-j)}\right)_{\substack{1 \leq i \leq d+N \\
1 \leq j \leq N}}\right)}{\Delta_{d+N}\left(q_{1}, \ldots, q_{d+N}\right)}
\end{aligned}
$$

\footnotetext{
${ }^{12}$ Given the set $(1, \ldots, M)$, and a subset $\left(i_{1}<\cdots<i_{d}\right)$, define

$$
\sigma_{M}\left(i_{1}, \ldots, i_{d}\right):=\#\left\{\begin{array}{c}
\text { transpositions needed to map } \\
\left(1<\cdots<i_{1}<\cdots<i_{d}<\cdots<M\right) \\
\text { into }\left(i_{1}, \ldots, i_{d}, 1,2, \ldots, \hat{i}_{1}, \ldots, \hat{i}_{d}, \ldots, M\right)
\end{array}\right\} .
$$
}




$$
\begin{aligned}
= & (-1)^{d(d-1) / 2} \prod_{i=1}^{d+N} q^{d x_{i}} \sum_{1 \leq i_{1}<\ldots<i_{d} \leq c}(-1)^{\sigma\left(i_{1}, \ldots, i_{d}\right)} \operatorname{det}\left(\begin{array}{c}
\tilde{\mathcal{P}}_{N-1}^{y_{j}}\left(q_{i_{1}}\right) \\
\vdots \\
\tilde{\mathcal{P}}_{N-1}^{y_{j}}\left(q_{i_{d}}\right)
\end{array}\right)_{1 \leq j \leq d} \\
& \times \frac{\Delta_{N}\left(q_{1}, \ldots, \widehat{q_{i_{1}}}, \ldots, \widehat{q_{i_{d}}}, \ldots, q_{d+N}\right)}{\Delta_{d+N}\left(q_{1}, \ldots, q_{d+N}\right)} \\
= & \frac{(-1)^{d(d-1) / 2}}{d !} \prod_{i=1}^{d+N} q^{d x_{i}} \sum_{1 \leq i_{1}, \ldots, i_{d} \leq c} \frac{\Delta_{q, d}^{\left(\mathbf{y}_{\text {cut }}\right)}\left(q_{i_{1}}, \ldots, q_{i_{d}}\right) \Delta_{d}\left(q_{i_{1}}, \ldots, q_{i_{d}}\right)}{\prod_{j-1}^{d} Q_{q}^{\prime}\left(q_{i_{j}}\right)} \\
= & \frac{(-1)^{d(d-1) / 2}}{d !}\left(\prod_{i=1}^{d+N} q^{d x_{i}}\right) \Omega_{q}(0,0),
\end{aligned}
$$

upon using the residue formula 86 for $\Omega_{q}(0,0)$ in the last equality.

Expressing the entries of inverse $\widetilde{H}^{-1}$ as integrals. Computing its entries $\widetilde{H}_{r, k}^{-1}$ will depend on whether $1 \leq r \leq d$ or $d+1 \leq r \leq d+N$. To do so, the determinant of the adjoint matrix will be expanded as a sum of the determinants of blocks taken from the first part of the matrix times the determinant of a complementary block of the second part. Then using in $\stackrel{*}{=}$ the second relation of Lemma 3.4 one finds for $1 \leq r \leq d$ and $1 \leq k \leq d+N$ :

$$
\begin{aligned}
& \widetilde{H}_{r, k}^{-1}=\frac{\prod_{\substack{i=1 \\
i \neq k}}^{d+N} q^{d x_{i}} \operatorname{adj}\left(\begin{array}{c}
\left(\tilde{\mathcal{P}}_{N-1}^{y_{j}}\left(q^{x_{i}}\right)\right)_{\substack{1 \leq i \leq c \\
1 \leq j \leq d}} \\
O_{d+b, d}
\end{array} \mid\left(q^{x_{i}(N-j)}\right)_{\substack{1 \leq i \leq d+N \\
1 \leq j \leq N}}\right)_{r, k}}{\operatorname{det} \widetilde{H}} \\
& =\frac{d !(-1)^{r+k+d(d-1) / 2}}{\Omega_{q}(0,0) q^{d x_{k}}} \sum_{1 \leq i_{1}<\cdots<i_{d-1} \leq c}(-1)^{\sigma} \\
& \times \operatorname{det}\left(\begin{array}{ccccc}
\tilde{\mathcal{P}}_{N-1}^{y_{1}}\left(q_{i_{1}}\right) & \ldots & \tilde{\mathcal{P}}_{N-1}^{y_{r}}\left(q_{i_{1}}\right) & \ldots & \tilde{\mathcal{P}}_{N-1}^{y_{d}}\left(q_{i_{1}}\right) \\
\vdots & & \vdots & & \vdots \\
\tilde{\mathcal{P}}_{N-1}^{y_{1}}\left(q_{i_{d-1}}\right) & \ldots & \left.\tilde{\mathcal{P}}_{N-1}^{y_{r}\left(q_{i_{d-1}}\right.}\right) & \ldots & \tilde{\mathcal{P}}_{N-1}^{y_{d}}\left(q_{i_{d-1}}\right)
\end{array}\right) \\
& \times \frac{\Delta_{N}\left(q_{1}, \ldots, \widehat{q_{k}}, \widehat{q_{i_{1}}}, \ldots, \widehat{q}_{i_{d-1}}, \ldots, q_{d+N}\right)}{(-1)^{k-1} \Delta_{d+N}\left(q_{k}, q_{1}, \ldots, \widehat{q}_{k}, \ldots, q_{d+N}\right)}
\end{aligned}
$$

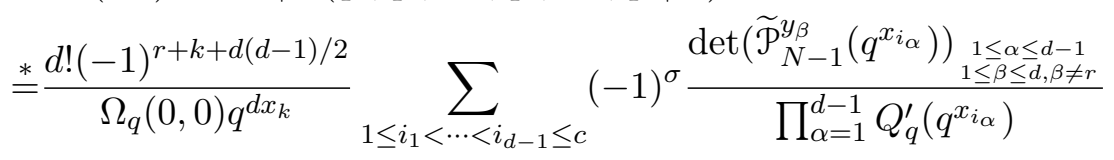

$$
\begin{aligned}
& \times \frac{(-1)^{\sigma+d(d-1) / 2} \Delta_{d}\left(q_{k}, q_{i_{1}}, \ldots, q_{i_{d-1}}\right)}{(-1)^{k-1} Q_{q}^{\prime}\left(q_{k}\right)} \\
& =\frac{d !(-1)^{r-1}}{(d-1) !} \frac{q^{-d x_{k}} F_{r}\left(q^{x_{k}}\right)}{Q_{q}^{\prime}\left(q^{x_{k}}\right) \Omega_{q}(0,0)}=(-1)^{r-1} d \oint_{\Gamma\left(q^{x_{k}}\right)} \frac{v^{-d} d v}{2 \pi \mathrm{i} Q_{q}(v)} \frac{F_{r}(v)}{\Omega_{q}(0,0)} \text {. }
\end{aligned}
$$


Also the last equality follows from replacing $\sum_{1 \leq i_{1}<\cdots<i_{d-1} \leq c}$ by $\sum_{1 \leq i_{1}, \ldots, i_{d-1} \leq c}$, which brings in the denominator $(d-1)$ !. Remember $F_{r}$ is the $d-1$-fold integral (85).

Using 88 above, we now show that for $1 \leq r \leq N$ the inverse $\widetilde{H}_{d+r, k}^{-1}$ has a multiple integral representation, as in the previous computation. Namely one uses in $\stackrel{*}{=}$ the first identity 54 (Lemma 3.4 and the definition 82 of $\Delta_{q, d}^{\left(\mathbf{y}_{\text {cut }}\right)}$. Also notice that the restriction $i_{\ell} \neq k$ in the sum appearing in $\stackrel{*}{=}$ can be removed, since $\Delta_{N-1}^{\widehat{N-r}}=0$ for $i_{\ell}=k$. In $\stackrel{* *}{=}$, one uses 55 (Lemma 3.4 and the terms containing $q^{x_{k}}$ can be rewritten as a contour integral about $q^{x_{k}}$, because of the presence of $Q_{q}^{\prime}\left(q^{x_{k}}\right)$ in the denominator. Finally, in $\stackrel{* * *}{=}$, one uses the definition 86) of $\Omega_{q}(v, z)$, thus yielding:

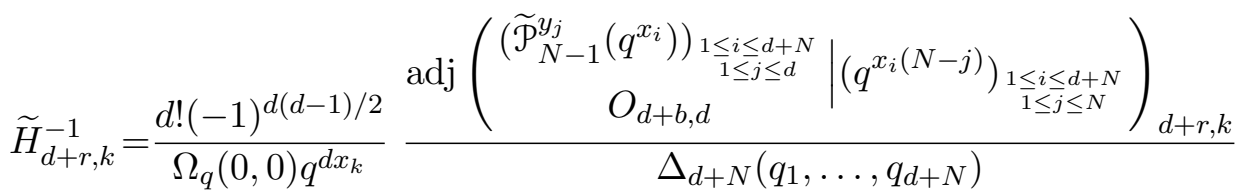

$$
\begin{aligned}
& \left.=\frac{d !(-1)^{d(d-1) / 2}}{\Omega_{q}(0,0) q^{d x_{k}}}(-1)^{d+r+k} \sum_{\substack{1 \leq i_{1}<\cdots<i_{d} \leq c \\
\text { all } \\
i_{\ell} \neq k}}(-1)^{\sigma} \operatorname{det}\left(\widetilde{\mathcal{P}}_{N-1}^{y_{\beta}}\left(q^{x_{i_{\alpha}}}\right)\right)\right)_{1 \leq \alpha, \beta \leq d} \\
& \times \frac{\Delta_{N-1}^{\widehat{N-r}}\left(q_{1}, \ldots, \widehat{q_{k}}, \widehat{q_{i_{1}}}, \ldots, \widehat{q_{i_{d}}}, \ldots, q_{d+N}\right)}{\Delta_{d+N}\left(q_{1}, \ldots, q_{d+N}\right)} \\
& \stackrel{*}{=} \frac{d !(-1)^{d(d+1) / 2+r+k}}{\Omega_{q}(0,0) q^{d x_{k}}} \sum_{1 \leq i_{1}<\cdots<i_{d} \leq c}(-1)^{\sigma} \Delta_{q, d}^{\left(\mathbf{y}_{\text {cut }}\right)}\left(q^{x_{i_{1}}}, \ldots, q^{x_{i_{d}}}\right) \\
& \times(-1)^{\frac{d(d+1)}{2}+\sigma+k-1} \frac{\Delta_{d+1}\left(q_{k}, q_{i_{1}}, \ldots, q_{i_{d}}\right) e_{r-1}\left(q_{1}, \ldots, \widehat{q_{k}}, \widehat{q_{i_{1}}}, \ldots, \widehat{q_{i_{d}}}, \ldots, q_{d+N}\right)}{\prod_{\alpha=1}^{d} Q_{q}^{\prime}\left(q^{x_{i_{\alpha}}}\right) Q_{q}^{\prime}\left(q_{k}\right)} \\
& \stackrel{* *}{=} \frac{1}{\Omega_{q}(0,0) q^{d x_{k}}} \sum_{1 \leq i_{1}<\cdots<i_{d} \leq c} \Delta_{q, d}^{\left(\mathbf{y}_{\text {cut }}\right)}\left(q^{x_{i_{1}}}, \ldots, q^{x_{i_{d}}}\right) \Delta_{d}\left(q^{x_{i_{1}}}, \ldots, q^{x_{i_{d}}}\right) \\
& \times \frac{1}{Q_{q}^{\prime}\left(q^{x_{k}}\right)} \prod_{\alpha=1}^{d} \frac{\left(q^{x_{k}}-q^{x_{i_{\alpha}}}\right)}{Q_{q}^{\prime}\left(q^{x_{i_{\alpha}}}\right)} \oint_{\Gamma_{\infty}} \frac{d z}{2 \pi \mathrm{i} z^{N-r+1}} \frac{Q_{q}(z)}{\left(z-q^{x_{k}}\right) \prod_{\ell=1}^{d}\left(z-q^{x_{i_{\ell}}}\right)} \\
& \stackrel{* * *}{=} \oint_{\Gamma_{q^{x} k}} \frac{d v}{2 \pi \mathrm{i} v^{d}} \oint_{\Gamma_{\infty}} \frac{d z}{2 \pi \mathrm{i} z^{N-r+1}} \frac{1}{(z-v)} \frac{Q_{q}(z)}{Q_{q}(v)} \frac{\Omega_{q}(v, z)}{\Omega_{q}(0,0)} \text {. }
\end{aligned}
$$

The computation of the $\widetilde{\psi}_{k}^{(m+1)}$-function, as defined in 76 of Proposition 6.1. comes in two parts. For $1 \leq r \leq N$, we have, using directly 90 and 80 ,

$$
\widetilde{\psi}_{d+r}^{(m+1)}(x)=\sum_{k=1}^{d+N}\left(\widetilde{H}^{-1}\right)_{d+r, k} h_{x_{k}-x}\left(q^{d+m}, \ldots, q^{d+N-1}\right) \mathbb{1}_{x_{k} \geq x}
$$




$$
\begin{aligned}
& =\left.q^{-x(d+m)} \sum_{k=1}^{d+N} \widetilde{H}_{d+r, k}^{-1} v^{d+m} \mathcal{P}_{N-m-1}\left(v q^{-x}\right)\right|_{v=q_{k}} \mathbb{1}_{x_{k} \geq x} \\
& =q^{-x(d+m)} \oint_{\Gamma\left(q^{x}, q^{x+1}, \ldots\right)} \frac{v^{m} d v}{2 \pi \mathrm{i}} \oint_{\Gamma_{\infty}} \frac{d z}{2 \pi \mathrm{i} z^{N-r+1}} \frac{\mathcal{P}_{N-m-1}\left(v q^{-x}\right)}{(z-v)} \frac{Q_{q}(z)}{Q_{q}(v)} \frac{\Omega_{q}(v, z)}{\Omega_{q}(0,0)}
\end{aligned}
$$

Similarly for $1 \leq r \leq d$, using (89), (76) and (85),

$$
\begin{aligned}
\widetilde{\psi}_{r}^{(m+1)}(x)= & \sum_{k=1}^{d+N}\left(\widetilde{H}^{-1}\right)_{r k} h_{x_{k}-x}\left(q^{d+m}, \ldots, q^{d+N-1}\right) \mathbb{1}_{x_{k} \geq x} \\
& =\frac{d(-1)^{r-1}}{q^{x(d+m)}} \sum_{\substack{1 \leq k \leq d+N \\
x_{k} \geq x}} q^{x_{k}(d+m)} \mathcal{P}_{N-m-1}\left(q^{x_{k}-x}\right) \oint_{\Gamma_{q} x_{k}} \frac{v^{-d} d v}{2 \pi \mathrm{i} Q_{q}(v)} \frac{F_{r}(v)}{\Omega_{q}(0,0)} \\
& =\frac{d(-1)^{r-1}}{q^{x(d+m)}} \oint_{\Gamma\left(q^{x+\mathbb{N}}\right)} \frac{v^{m} \mathcal{P}_{N-m-1}\left(v q^{-x}\right) d v}{2 \pi \mathrm{i} Q_{q}(v)} \frac{F_{r}(v)}{\Omega_{q}(0,0)}
\end{aligned}
$$

Computing each of the terms $\mathbb{K}_{q}^{(i)}$ in the kernel $(\mathbf{8 3})$ for $i=0,1,2$ and for $0 \leq m, n \leq N$. Indeed, using $(80)$, one finds for $(i)$ of $(78)$ :

$$
\begin{aligned}
\mathbb{K}_{q}^{(0)}(m, x ; n, y) & =h_{y-x}\left(q^{d+m}, \ldots, q^{d+n-1}\right) \mathbb{1}_{n>m} \mathbb{1}_{y \geq x} \\
& =\left.z^{d+m} \mathcal{P}_{n-m-1}(z)\right|_{z=q^{y-x}} \mathbb{1}_{n>m} \mathbb{1}_{y \geq x} \\
& =\left.z^{d+m} \prod_{i=0}^{n-m-2} \frac{1-z q^{i+1}}{1-q^{i+1}}\right|_{z=q^{y-x}} \mathbb{1}_{n>m} \mathbb{1}_{y \geq x} \\
& =\left.q^{(d+m)(y-x)} \frac{(z q ; q)_{n-m-1}}{(q ; q)_{n-m-1}}\right|_{z=q^{y-x}} \mathbb{1}_{n>m} \mathbb{1}_{y \geq x} .
\end{aligned}
$$

For $1 \leq k \leq N, m \geq 0$, using formula (91) for $r=N-k+1$ and using Petrov's expression (39), one checks for $(i i)$ of (78):

$$
\begin{aligned}
\mathbb{K}_{q}^{(1)}(m, x ; n, y)= & q^{d y} \sum_{k=1}^{n} \psi_{d+N-k+1}^{(m+1)}(x) q^{(k-1) y} \prod_{r=n+1}^{N}\left(1-q^{r-k}\right) \\
= & q^{d(y-x)-x m} \oint_{\Gamma\left(q^{x+\mathbb{N}}\right)} \frac{v^{m} d v}{2 \pi \mathrm{i}} \oint_{\Gamma_{\infty}} \frac{d z}{2 \pi \mathrm{i} z} \frac{\mathcal{P}_{N-m-1}\left(v q^{-x}\right)}{(z-v)} \frac{Q_{q}(z)}{Q_{q}(v)} \frac{\Omega_{q}(v, z)}{\Omega_{q}(0,0)} \\
& \times z \sum_{k=1}^{n} z^{-k} q^{(k-1) y} \prod_{r=n+1}^{N}\left(1-q^{r-k}\right)
\end{aligned}
$$




$$
\begin{aligned}
& =q^{d(y-x)-x m} \oint_{\Gamma\left(q^{x+\mathbb{N}}\right)} \frac{v^{m} d v}{2 \pi \mathrm{i}} \oint_{\Gamma_{\infty}} \frac{\Phi_{q}\left(q^{y} z^{-1}\right) d z}{2 \pi \mathrm{i} z} \frac{\mathcal{P}_{N-m-1}\left(v q^{-x}\right)}{(z-v)} \frac{Q_{q}(z)}{Q_{q}(v)} \frac{\Omega_{q}(v, z)}{\Omega_{q}(0,0)} \\
& \stackrel{*}{=} q^{(y-x)(d+m)} \oint_{\Gamma\left(q^{x-y+\mathbb{N}}\right)} \frac{v^{m} d v}{2 \pi \mathrm{i}} \oint_{\Gamma_{\infty}} \frac{\Phi_{q}\left(z^{-1}\right) d z}{2 \pi \mathrm{i} z} \frac{\mathcal{P}_{N-m-1}\left(v q^{y-x}\right)}{(z-v)} \frac{Q_{q}\left(z q^{y}\right)}{Q_{q}\left(v q^{y}\right)} \frac{\Omega_{q}\left(v q^{y}, z q^{y}\right)}{\Omega_{q}(0,0)} .
\end{aligned}
$$

This last equality $\stackrel{*}{=}$ is obtained by the substitution $v \mapsto v q^{y}$ and $z \mapsto z q^{y}$. Substituting 92 for $\widetilde{\psi}_{r}^{(m+1)}$ and 80 for $h_{y-y_{r}}$, and using (84), one finds for (iii) of $(78)$ :

$$
\begin{aligned}
\mathbb{K}_{q}^{(2)}(m, x ; n, y)= & \sum_{1 \leq r \leq d} \widetilde{\psi}_{r}^{(m+1)}(x) h_{y-y_{r}}\left(q^{d}, \ldots, q^{d+n-1}\right) \\
= & \frac{d}{q^{x(d+m)}} \oint_{\Gamma\left(q^{x+\mathbb{N}}\right)} \frac{v^{m} \mathcal{P}_{N-m-1}\left(v q^{-x}\right) d v}{2 \pi \mathrm{i} Q_{q}(v)} \\
& \times \sum_{1 \leq r \leq d}(-1)^{r-1} h_{y-y_{r}}(\bullet) \frac{F_{r}(v)}{\Omega_{q}(0,0)} \\
= & \frac{d}{q^{x(d+m)}} \oint_{\Gamma\left(q^{x+\mathbb{N}}\right)} \frac{v^{m} \mathcal{P}_{N-m-1}\left(v q^{-x}\right) d v}{2 \pi \mathrm{i} Q_{q}(v)} \frac{\widetilde{\Omega}_{q}(v, y)}{\Omega_{q}(0,0)}
\end{aligned}
$$

Expression $\mathbb{K}_{q}^{(2)}$ as in the kernel 83 follows by the substitution $v \mapsto v q^{y}$. This ends the proof of Theorem 6.2 .

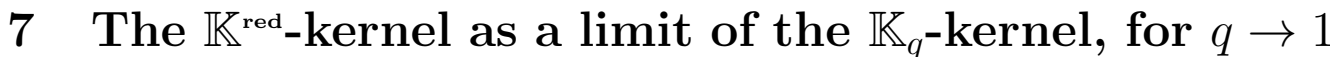

Before stating Proposition 7.1, we consider the limits for $q \rightarrow 1$ of the expressions (82), yielding by Lemma 2.1 the corresponding expressions (51):

$$
\begin{aligned}
\lim _{q \rightarrow 1} \Delta_{q, d}^{\left(\mathbf{y}_{\text {cut }}\right)}\left(q^{u_{1}}, \ldots, q^{u_{d}}\right) & =\Delta_{d}^{\left(\mathbf{y}_{\text {cut }}\right)}\left(u_{1}, \ldots, u_{d}\right) \\
\lim _{q \rightarrow 1} \widetilde{\Delta}_{q, d}^{\left(\mathbf{y}_{\text {cut }}\right)}\left(w ; u_{2}, \ldots, u_{d}\right) & =\widetilde{\Delta}_{d}^{\left(\mathbf{y}_{\text {cut }}\right)}\left(w ; u_{2}, \ldots, u_{d}\right) \\
\lim _{q \rightarrow 1} \widetilde{\Delta}_{q, d, n}^{\left(\mathbf{y}_{\text {cut }}\right)}\left(y ; u_{2}, \ldots, u_{d}\right) & =\widetilde{\Delta}_{d, n}^{\left(\mathbf{y}_{\text {cut }}\right)}\left(y ; u_{2}, \ldots, u_{d}\right) .
\end{aligned}
$$

The limiting kernel $\mathbb{K}^{\text {red }}$ in Proposition 7.1 is expressed as a $d+2$-fold integral, where -remember- $d$ is the sum of the sizes of the cuts below. Incidentally, the form (95) of the kernel in this proposition is the most convenient one to show that the kernel $\mathbb{K}^{\text {red }}$ is the inverse Kasteleyn matrix, up to some trivial conjugation; see [3]. 
Proposition 7.1 The limiting kernel for $q \rightarrow 1$ has the following form for $d \geq 0$, and $(m, x),(n, y) \in \mathbf{P}$ :

$$
\begin{aligned}
& \mathbb{K}^{r e d}(m, x ; n, y)=:\left(\mathbb{K}_{0}+\mathbb{K}_{1}+\mathbb{K}_{2}\right)(m, x ; n, y) \\
& =-\frac{(y-x+1)_{n-m-1}}{(n-m-1) !} \mathbb{1}_{n>m} \mathbb{1}_{y \geq x}+\oint_{\Gamma(x+\mathbb{N})} \frac{d v}{2 \pi \mathrm{i}}\left(\frac{(v-x+1)_{N-m-1}}{(N-m-1) ! Q(v)}\right) \\
& \quad \times\left(\oint_{\Gamma_{\infty}} \frac{d z}{2 \pi \mathrm{i}(z-v)}\left(\frac{(N-n) ! Q(z)}{(z-y)_{N-n+1}}\right) \frac{\Omega_{\mathcal{R}}(v, z)}{\Omega_{\mathcal{R}}(0,0)}\right. \\
& \left.\quad+d \oint_{\Gamma_{0}} \frac{d w}{2 \pi \mathrm{i} w^{y+1}(1-w)^{n}} \frac{\widetilde{\Omega}_{\mathcal{R}}^{(1)}(v, w)}{\Omega_{\mathcal{R}}(0,0)}\right),
\end{aligned}
$$

where, using the expressions $\Delta_{d}^{\left(\mathbf{y}_{\text {cut }}\right)}$ and $\widetilde{\Delta}_{d}^{\left(\mathbf{y}_{\text {cut }}\right)}$ in 51 ), (for later use $\widetilde{\Omega}_{\mathcal{R}}(v, w)$ is defined below with $P(z)$ as in (2) and for brevity, set $u=\left(u_{1}, \ldots, u_{d}\right)$ )

$$
\begin{gathered}
\Omega_{\mathcal{R}}(v, z):=\left(\prod_{\alpha=1}^{d} \oint_{\Gamma(\mathcal{R})} \frac{d u_{\alpha}}{2 \pi \mathrm{i} Q\left(u_{\alpha}\right)} \frac{v-u_{\alpha}}{z-u_{\alpha}}\right) \Delta_{d}(u) \Delta_{d}^{\left(\mathbf{y}_{c u t}\right)}(u) \\
\begin{aligned}
\widetilde{\Omega}_{\mathcal{R}}^{(1)}(v, z):=P(z) \widetilde{\Omega}_{\mathcal{R}}(v, z):=\left(\prod_{\alpha=2}^{d} \oint_{\Gamma(\mathcal{R})} \frac{d u_{\alpha}}{2 \pi \mathrm{i} Q\left(u_{\alpha}\right)}\right) \Delta_{d}\left(v, u_{2}, \ldots, u_{d}\right) \\
\times \widetilde{\Delta}_{d}^{\left(\mathbf{y}_{c u t}\right)}\left(z ; u_{2}, \ldots, u_{d}\right),
\end{aligned}
\end{gathered}
$$

where $\Omega_{\mathcal{R}}(v, z)=1$ for $d=0$, as before, and $\widetilde{\Omega}_{\mathcal{R}}^{(1)}(v, w)=w^{y_{1}}$ for $d=1$.

Corollary 7.2 Given an hexagon $\mathbf{P}$, with $\ell-1$ cuts on top and none at the bottom, the kernel $\mathbb{K}^{\text {red }}$ reads

$$
\begin{aligned}
& \mathbb{K}^{\text {red }}(m, x ; n, y)=-\frac{(y-x+1)_{n-m-1}}{(n-m-1) !} \mathbb{1}_{n>m} \mathbb{1}_{y \geq x} \\
& \quad+\frac{(N-n) !}{(N-m-1) !} \oint_{\Gamma(x+\mathbb{N})} \frac{d v}{2 \pi \mathrm{i}} \oint_{\Gamma_{\infty}} \frac{d z}{2 \pi \mathrm{i}(z-v)}\left(\frac{(v-x+1)_{N-m-1} Q(z)}{(z-y)_{N-n+1} Q(v)}\right),
\end{aligned}
$$

where (setting $a:=n_{1}+n_{2}$ )

(i) $Q(z)$ as in [2) for $\ell>1$. (Petrov [38])

(ii) $Q(z)=(z-a+1)_{c}(z+c+1)_{b}$, for $\ell=1$ (hexagon $\left.(a, b, c)\right)$.)(Johansson [20])

Proof of Proposition 7.1: Referring to the kernel (83) in Theorem 6.2, we first have $\lim _{q \rightarrow 1} \mathbb{K}_{q}^{(0)}=\mathbb{K}_{0}$. The other terms require some argumentation.

Next we prove $\lim _{q \rightarrow 1} \mathbb{K}_{q}^{(1)}=\mathbb{K}_{1}$. The term $\mathbb{K}_{q}^{(1)}$ can be expressed as follows, 
taking into account the roots $v=q^{x_{j}-y}$ of $Q_{q}\left(v q^{y}\right)$ and the integration contour $\Gamma\left(q^{x-y+\mathbb{N}}\right)$,

$$
\mathbb{K}_{q}^{(1)}(m, x ; n, y)=\oint_{\Gamma_{\infty}} \frac{d z}{2 \pi \mathrm{i} z} \sum_{\substack{1 \leq j \leq d+N \\ x_{j} \geq x}} \mathcal{R}_{j}(z),
$$

where

$$
\begin{aligned}
\mathcal{R}_{j}(z):= & \operatorname{Res}_{v=q^{x_{j}-y}} v^{m} \frac{\Phi_{q}\left(z^{-1}\right) \mathcal{P}_{N-m-1}\left(v q^{y-x}\right) Q_{q}\left(z q^{y}\right)}{(z-v) Q_{q}\left(v q^{y}\right)} \frac{\Omega_{q}\left(v q^{y}, z q^{y}\right)}{\Omega_{q}(0,0)} \\
= & q^{m\left(x_{j}-y\right)} \Phi_{q}\left(z^{-1}\right) \mathcal{P}_{N-m-1}\left(q^{x_{j}-x}\right) \prod_{\substack{1 \leq r \leq d+N \\
r \neq j}} \frac{z-q^{x_{r}-y}}{q^{x_{j}-y}-q^{x_{r}-y}} \frac{\Omega_{q}\left(q^{x_{j}}, z q^{y}\right)}{\Omega_{q}(0,0)} \\
\stackrel{*}{=} & \sum_{\substack{1 \leq i_{1}, \ldots, i_{d} \leq c \\
j \notin\left(i_{1}, \ldots, i_{d}\right)}} \frac{q^{m\left(x_{j}-y\right)} \mathcal{P}_{N-m-1}\left(q^{x_{j}-x}\right)}{\prod_{\substack{1 \leq r \leq d+N \\
r \neq j}}\left(q^{x_{j}-y}-q^{x_{r}-y}\right)}(\star) \\
& \times \Phi_{q}\left(z^{-1}\right) \prod_{\substack{1 \leq r \leq d+N \\
r \neq j, i_{1}, \ldots, i_{d}}}\left(z-q^{x_{r}-y}\right) \quad(\star \star) \\
& \times \frac{\Delta_{q, d}^{\left(\mathbf{y}_{\text {cut }}\right)}\left(q^{x_{i_{1}}}, \ldots, q^{x_{i}}\right) \Delta_{d}\left(q^{x_{i_{1}}}, \ldots, q^{x_{i_{d}}}\right)}{\Omega_{q}(0,0)} \prod_{\alpha=1}^{d} \frac{q^{x_{j}-y}-q^{x_{i_{\alpha}}-y}}{Q_{q}^{\prime}\left(q^{\left.x_{i_{\alpha}}\right)}\right.} \\
= & \sum_{\substack{1 \leq i_{1}, \ldots, i_{d} \leq c \\
j \notin\left(i_{1}, \ldots, i_{d}\right)}}((\star \star \star) \times(\star \star) \times(\star \star \star)) .
\end{aligned}
$$

Equality $\stackrel{*}{=}$ is obtained by inserting the expression $(86)$ for $\Omega_{q}(v, z)$ into the formula preceding $\stackrel{*}{=}$. One also notices that the term $\prod_{\alpha=1}^{d}\left(z-q^{x_{i_{\alpha}}-y}\right)$ in the denominator of $\Omega_{q}\left(q^{x_{j}}, z q^{y}\right)$ cancels $d$ terms in the product $\prod_{\substack{1 \leq r \leq d+N \\ r \neq j}}\left(z-q^{x_{r}-y}\right)$, yielding equality $(\stackrel{*}{=})$. Also $j$ can be taken different from $i_{1}, \ldots, i_{d}$, because if $j$ would figure in that set the term would vanish.

Now we let $q \rightarrow 1$. Since the terms $(\star)$ and $(\star \star \star)$ do not depend on $z$, the integration in (98) will act on term $(\star \star)$ only. So, at first for given $x_{j}$, we have, using Lemma 2.1, the estimate

$$
\begin{aligned}
(\star) & \simeq(q-1)^{1-N-d} \frac{\left(x_{j}-x+1\right)_{N-m-1}}{(N-m-1) ! \prod_{\substack{1 \leq r \leq d+N \\
r \neq j}}\left(x_{j}-x_{r}\right)} \\
& =(q-1)^{1-N-d} \frac{\left(x_{j}-x+1\right)_{N-m-1}}{(N-m-1) ! Q^{\prime}\left(x_{j}\right)} .
\end{aligned}
$$


Next, again using Lemma 2.1, we have for $q \sim 1$,

$$
\begin{aligned}
& \oint_{\Gamma_{\infty}} \frac{d z}{2 \pi \mathrm{i} z}(\star \star) \\
& =\oint_{\Gamma_{\infty}} \frac{d z}{2 \pi \mathrm{i} z} \Phi_{q}\left(z^{-1}\right) \prod_{\substack{1 \leq r \leq d+N \\
r \neq j, i_{1}, \ldots, i_{d}}}\left(z-q^{x_{r}-y}\right) \\
& \simeq(q-1)^{N-1}(N-n) ! \oint_{\Gamma_{\infty}} \frac{d z}{2 \pi \mathrm{i}(z-y)_{N-n+1}} \prod_{\substack{1 \leq r \leq d+N \\
r \neq j, i_{1}, \ldots, i_{d}}}\left(z-x_{r}\right) \\
& \simeq(q-1)^{N-1}(N-n) ! \oint_{\Gamma_{\infty}} \frac{d z Q(z)}{2 \pi \mathrm{i}(z-y)_{N-n+1}\left(z-x_{j}\right)} \frac{1}{\prod_{\alpha=1}^{d}\left(z-x_{i_{\alpha}}\right)} .
\end{aligned}
$$

Next we need to estimate $(\star \star \star)$. From (94), combined with some of the limits in Lemma 2.1, one finds the estimate

$$
\begin{aligned}
& \Delta_{d}\left(q^{x_{i_{1}}}, \ldots, q^{x_{i_{d}}}\right) \Delta_{q, d}^{\left(\mathbf{y}_{\text {cut }}\right)}\left(q^{x_{i_{1}}}, \ldots, q^{x_{i_{d}}}\right) \prod_{\alpha=1}^{d} \frac{q^{x_{j}-y}-q^{x_{i_{\alpha}}-y}}{Q_{q}^{\prime}\left(q^{x_{i_{\alpha}}}\right)} \\
& \simeq(q-1)^{-d\left(N+\frac{d-1}{2}-1\right)} \Delta_{d}\left(x_{i_{1}}, \ldots, x_{i_{d}}\right) \Delta_{d}^{\left(\mathbf{y}_{\mathrm{cut}}\right)}\left(x_{i_{1}}, \ldots, x_{i_{d}}\right) \prod_{\alpha=1}^{d} \frac{x_{j}-x_{i_{\alpha}}}{Q^{\prime}\left(x_{i_{\alpha}}\right)} .
\end{aligned}
$$

and thus, when $q \rightarrow 1$, the residue version of expression $\Omega_{\mathcal{R}}(0,0)$ defined in 86 tends to the residue version of $(96)$, modulo a power of $(q-1)$ :

$$
\begin{aligned}
\Omega_{q}(0,0) & =\sum_{1 \leq i_{1}, \ldots, i_{d} \leq c} \frac{\Delta_{d}\left(q^{x_{i_{1}}}, \ldots, q^{x_{i}}\right) \Delta_{q, d}^{\left(\mathbf{y}_{\mathrm{cut}}\right)}\left(q^{x_{i_{1}}}, \ldots, q^{x_{i_{d}}}\right)}{\prod_{\alpha=1}^{d} Q_{q}^{\prime}\left(q^{x_{i_{\alpha}}}\right)} \\
& \simeq(q-1)^{-d\left(N+\frac{d-1}{2}\right)} \sum_{1 \leq i_{1}, \ldots, i_{d} \leq c} \frac{\Delta_{d}\left(x_{i_{1}}, \ldots, x_{i_{d}}\right) \Delta_{d}^{\left(\mathbf{y}_{\mathrm{cut}}\right)}\left(x_{i_{1}}, \ldots, x_{i_{d}}\right)}{d} \prod_{\alpha=1}^{d} Q^{\prime}\left(x_{i_{\alpha}}\right) \\
& =(q-1)^{-d\left(N+\frac{d-1}{2}\right)} \Omega_{\mathcal{R}}(0,0) .
\end{aligned}
$$

Upon using (94), it follows that the last line $(\star \star \star)$ of formula $(99)$ is estimated by

$$
\star \star \star \simeq \frac{(q-1)^{d}}{\Omega_{\mathcal{R}}(0,0)}\left(\prod_{\alpha=1}^{d} \frac{\left(x_{j}-x_{i_{\alpha}}\right)}{Q^{\prime}\left(x_{i_{\alpha}}\right)}\right) \Delta_{d}\left(x_{i_{1}}, \ldots, x_{i_{d}}\right) \Delta_{d}^{\left(\mathbf{y}_{\mathrm{cut}}\right)}\left(x_{i_{1}}, \ldots, x_{i_{d}}\right) .
$$

Multiply the three contributions (100), (101) and (104) together and do the summation, in which the requirement $j \notin\left(i_{1}, \ldots, i_{d}\right)$ can be removed; indeed 
whenever $j \in\left(i_{1}, \ldots, i_{d}\right)$, the sum below would automatically vanish. So we find for each $x_{j} \geq x$

$$
\begin{aligned}
& \lim _{q \rightarrow 1} \oint_{\Gamma_{\infty}} \frac{d z}{2 \pi \mathrm{i} z} \mathcal{R}_{j}(z) \\
&=\frac{(N-n) !}{(N-m-1) !} \frac{\left(x_{j}-x+1\right)_{N-m-1}}{Q^{\prime}\left(x_{j}\right)} \oint_{\Gamma_{\infty}} \frac{d z Q(z)}{2 \pi \mathrm{i}(z-y)_{N-n+1}\left(z-x_{j}\right)} \\
& \times \sum_{1 \leq i_{1}, \ldots, i_{d} \leq c}\left(\prod_{\alpha=1}^{d} \frac{\left(x_{j}-x_{i_{\alpha}}\right)}{\left(z-x_{i_{\alpha}}\right) Q^{\prime}\left(x_{i_{\alpha}}\right)}\right) \frac{\Delta_{d}\left(x_{i_{1}}, \ldots, x_{i_{d}}\right) \Delta_{d}^{\left(\mathbf{y}_{\mathrm{cut}}\right)}\left(x_{i_{1}}, \ldots, x_{i_{d}}\right)}{\Omega_{\mathcal{R}}(0,0)} \\
&= \frac{(N-n) !}{(N-m-1) !} \frac{\left(x_{j}-x+1\right)_{N-m-1}}{Q^{\prime}\left(x_{j}\right)} \oint_{\Gamma_{\infty}} \frac{d z Q(z)}{2 \pi \mathrm{i}(z-y)_{N-n+1}\left(z-x_{j}\right)} \frac{\Omega_{\mathcal{R}}\left(x_{j}, z\right)}{\Omega_{\mathcal{R}}(0,0)} .
\end{aligned}
$$

So we conclude, upon summing the $v$-residues,

$$
\begin{aligned}
\lim _{q \rightarrow 1} \mathbb{K}_{q}^{(1)}(m, x ; n, y)=\frac{(N-n) !}{(N-m-1) !} \oint_{\Gamma(x+\mathbb{N})} & \frac{d v}{2 \pi \mathrm{i}} \oint_{\Gamma_{\infty}} \frac{d z}{2 \pi \mathrm{i}} \frac{(v-x+1)_{N-m-1}}{(z-v)(z-y)_{N-n+1}} \\
& \times \frac{Q(z)}{Q(v)} \frac{\Omega_{\mathcal{R}}(v, z)}{\Omega_{\mathcal{R}}(0,0)}=\mathbb{K}_{1}(m, x ; n, y) .
\end{aligned}
$$

Consider now the $\mathbb{K}_{q}^{(2)}$ part of the kernel 83 , given in residue form as

$$
\begin{aligned}
\mathbb{K}_{q}^{(2)}(m, x ; n, y) & =\frac{d}{q^{y(2 d+N-1)}} \oint_{\Gamma_{\Gamma\left(q^{x-y+N}\right)}} \frac{d v v^{m} \mathcal{P}_{N-m-1}\left(v q^{y-x}\right)}{2 \pi i \prod_{1}^{d+N}\left(v-q^{x_{i}-y}\right)} \frac{\widetilde{\Omega}_{q}\left(v q^{y}, y\right)}{\Omega_{q}(0,0)} \\
& =\frac{d}{q^{y d}} \sum_{x_{i} \geq x} \frac{q^{m\left(x_{i}-y\right)} \mathcal{P}_{N-m-1}\left(q^{x_{i}-x}\right)}{\widetilde{\Omega}_{q}\left(q^{x_{i}}, y\right)} \\
Q_{q}^{\prime}\left(q^{x_{i}}\right) &
\end{aligned}
$$

with $\widetilde{\Omega}_{q}\left(q^{x_{i}}, y\right)$ given in 86, , from which it follows that, upon using 51, , 82 , (80), 91), Lemma 2.1 and (94),

$$
\begin{aligned}
\widetilde{\Omega}_{q}\left(q^{x_{j}}, y\right) & =\sum_{1 \leq i_{2}, \ldots, i_{d} \leq c} \frac{\Delta_{d}\left(q^{x_{j}}, q^{x_{i_{2}}}, \ldots, q^{x_{i}}\right) \widetilde{\Delta}_{q, d, n}^{\left(\mathbf{y}_{\text {cut }}\right)}\left(q^{y}, q^{x_{i_{2}}}, \ldots, q^{x_{i_{d}}}\right)}{\prod_{\alpha=2}^{d} Q_{q}^{\prime}\left(q^{\left.x_{i_{\alpha}}\right)}\right.} . \\
= & \frac{1}{(q-1)^{(N-1+d / 2)(d-1)}}\left(\oint_{\Gamma_{0}} \frac{d w}{2 \pi \mathrm{i} w^{y+1}(1-w)^{n}}\right) \\
& \times\left(\prod_{\alpha=2}^{d} \oint_{\Gamma\left(x_{1}, \ldots, x_{c}\right)} \frac{d u_{\alpha}}{2 \pi \mathrm{i} Q\left(u_{\alpha}\right)}\right) \Delta_{d}\left(x_{j}, u_{2}, \ldots, u_{d}\right) \widetilde{\Delta}_{d}^{\left(\mathbf{y}_{\text {cut }}\right)}\left(w ; u_{2}, \ldots, u_{d}\right) \\
= & \frac{1}{(q-1)^{(N-1+d / 2)(d-1)}} \oint_{\Gamma_{0}} \frac{d w \widetilde{\Omega}_{\mathcal{R}}^{(1)}\left(x_{j}, w\right)}{2 \pi \mathrm{i} w^{y+1}(1-w)^{n}},
\end{aligned}
$$


and thus, combined with the estimate 103 , this gives

$$
\frac{\widetilde{\Omega}_{q}\left(q^{x_{j}}, y\right)}{\Omega_{q}(0,0)}=(q-1)^{d+N-1} \oint_{\Gamma_{0}} \frac{d w}{2 \pi \mathrm{i} w^{y+1}(1-w)^{n}} \frac{\widetilde{\Omega}_{\mathcal{R}}^{(1)}\left(x_{j}, w\right)}{\Omega_{\mathcal{R}}(0,0)} .
$$

Inserting this formula into (105), and using the limits (40), we find

$$
\begin{aligned}
\lim _{q \rightarrow 1} \mathbb{K}_{q}^{(2)}(m, x ; n, y)= & \frac{d}{(N-m-1) !} \oint_{\Gamma(x+\mathbb{N})} \frac{d v}{2 \pi \mathrm{i} Q(v)}(v-x+1)_{N-m-1} \\
& \quad \times \oint_{\Gamma_{0}} \frac{d w}{2 \pi \mathrm{i} w^{y+1}(1-w)^{n}} \frac{\widetilde{\Omega}_{\mathcal{R}}^{(1)}(v, z)}{\Omega_{\mathcal{R}}(0,0)}=\mathbb{K}_{2}(m, x ; n, y),
\end{aligned}
$$

yielding the third part $\mathbb{K}_{2}$ of the kernel (95), thus proving Proposition 7.1 .

Proof of Corollary 7.2. Setting $d=0$ in the kernel (95) leads to formulas (97). Expanding $1 /(z-v)=\sum v^{j} / z^{j+1}$ formula (97) can be written as a finite sum $\sum p_{j}(x) q_{j}(y)$, with $p, q$ polynomials. It is unclear in case (ii) how this relates to the extended Hahn kernel in [20].

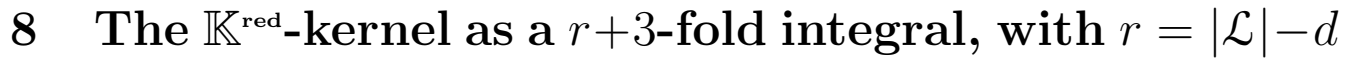

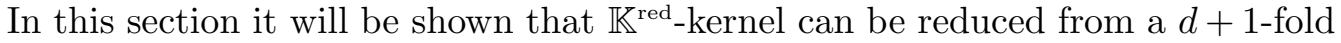
integral (95) to a $r+3$-fold integral (9), where (remember!) $d$ is the total sizes of the lower-cuts; this will establish Theorem 1.1 .

This reduction will be instrumental in performing the asymptotic analysis on $\mathbb{L}^{\text {blue }}$ for the two-cut model in [3], when all the sides and cuts of the polygon $\mathbf{P}$ tend to $\infty$, while $r$ and $\rho$ remain finite. Remember that in the two-cut model $r:=|\mathcal{L}|-d$ happens to be the number of blue dots on the oblique lines $\{\eta=$ $\left.m_{1}+k\right\}$ within the strip $\rho$.

Proposition 7.1 expresses the kernel in terms of two multiple integrals $\Omega_{\mathcal{R}}$ and $\widetilde{\Omega}_{\mathcal{R}}$ as in $(96)$, which become after substituting 52 and 53 for $\Delta_{d}^{\left(\mathbf{y}_{\text {cut }}\right)}$ and $\widetilde{\Delta}_{d}^{\left(\mathbf{y}_{\text {cut }}\right)}$ in these expressions,

$$
\begin{aligned}
\Omega_{\mathcal{R}}(v, z)= & C_{N, d}\left(\prod_{\alpha=1}^{d} \oint_{\Gamma(\mathcal{R})} \frac{d u_{\alpha} P\left(u_{\alpha}\right)}{2 \pi \mathrm{i} Q\left(u_{\alpha}\right)} \frac{v-u_{\alpha}}{z-u_{\alpha}}\right) E_{g}^{\left(\mathbf{y}_{\text {cut }}\right)}(u) \Delta_{d}(u)^{2} \\
\widetilde{\Omega}_{\mathcal{R}}(v, z):= & C_{N, d}\left(\prod_{\alpha=2}^{d} \oint_{\Gamma(\mathcal{R})} \frac{d u_{\alpha} P\left(u_{\alpha}\right)}{2 \pi \mathrm{i} Q\left(u_{\alpha}\right)} \frac{v-u_{\alpha}}{z-u_{\alpha}}\right) \\
& \times E_{g}^{\left(\mathbf{y}_{\text {cut }}\right)}\left(z, u_{2}, \ldots, u_{d}\right) \Delta_{d}\left(z, u_{2}, \ldots, u_{d}\right)^{2} .
\end{aligned}
$$


Define the same expressions, but with the contours $\Gamma(\mathcal{R})$ replaced by $\Gamma(\mathcal{L})$ :

$$
\Omega_{\mathcal{L}}(v, z) \text { and } \widetilde{\Omega}_{\mathcal{L}}(v, z),
$$

with $\Omega_{\mathcal{R}}(v, z)=1$ for $d=0$ and $\widetilde{\Omega}_{\mathcal{R}}(v, z)=1$ for $d=1$ and similarly for $\Omega_{\mathcal{L}}(v, z), \widetilde{\Omega}_{\mathcal{L}}(v, z)$. We now state :

Proposition 8.1 For $(m, x),(n, y) \in \mathbf{P}$, the kernel for the $\mathbb{K}^{\text {red }}$-process takes on two different forms, a first one involving (107) and a second one involving (108),

$$
\begin{aligned}
\mathbb{K}^{r e d}(m, x ; n, y) & \\
= & \mathbb{K}_{0}(m, x ; n, y)+\frac{(N-n) !}{(N-m-1) !} \oint_{\Gamma(x+\mathbb{N})} \frac{d v(v-x+1)_{N-m-1}}{2 \pi \mathrm{i} Q(v)} \\
& \times\left(\oint_{\Gamma_{\infty}} \frac{d z Q(z)}{2 \pi \mathrm{i}(z-v)(z-y)_{N-n+1}} \frac{\Omega_{\mathcal{R}}(v, z)+d(z-v) \frac{P(z)}{Q(z)} \widetilde{\Omega}_{\mathcal{R}}(v, z)}{\Omega_{\mathcal{R}}(0,0)}\right. \\
= & \left.\mathbb{K}_{0}(m, x ; n, y)+\frac{(N-n) !}{(N-m-1) !} \oint_{\Gamma(x+\mathbb{N})} \frac{d v(v-x+1)_{N-m-1}}{2 \pi \mathrm{i} Q(v)} \oint_{\Gamma_{\tau}} \frac{d z P(z)}{2 \pi \mathrm{i}(z-y)_{N-n+1}} \frac{\widetilde{\Omega}_{\mathcal{R}}(v, z)}{\Omega_{\mathcal{R}}(0,0)}\right) \\
& \times\left(\oint_{\Gamma_{\infty}} \frac{d z Q(z)}{2 \pi \mathrm{i}(z-v)(z-y)_{N-n+1}} \frac{\Omega_{\mathcal{L}}(v, z)}{\Omega_{\mathcal{L}}(0,0)}+d \oint_{\Gamma_{\tau}} \frac{d z P(z)}{2 \pi \mathrm{i}(z-y)_{N-n+1}} \frac{\widetilde{\Omega}_{\mathcal{L}}(v, z)}{\Omega_{\mathcal{L}}(0,0)}\right),
\end{aligned}
$$

with the $\Omega$ 's and $\widetilde{\Omega}$ 's defined in (108) and the contour $\Gamma_{\tau}$ defined in $(10)$. Contour $\Gamma_{\infty}=\Gamma(v ; y, \ldots, y-N+n)$ is a large circle about all the poles of the integrand.

To prove Proposition 8.1, we need the following Lemma:

Lemma 8.2 Given a rational function $R(u)$ with possibly poles within a contour $\Gamma$ and a point $z$ not within $\Gamma$, not a pole of $R(u)$ and given a symmetric polynomial $S(\mathbf{u})$, with $\mathbf{u}=\left(u_{1}, \ldots, u_{\ell}\right)$. Then for $0 \leq k-1 \leq \ell$, we have (the notation $\Gamma \cup z$ refers to the contour $\Gamma$, deformed so as to contain $z \in \mathbb{C})$, setting $\mathbf{u}=\left(u_{1}, \ldots, u_{\ell}\right)$,

$$
\begin{aligned}
& \left(\prod_{\alpha=1}^{\ell} \oint_{\Gamma \cup z} \frac{d u_{\alpha} R\left(u_{\alpha}\right)}{2 \pi \mathrm{i}\left(u_{\alpha}-z\right)}\right) \Delta_{\ell}^{2}(\mathbf{u}) S(\mathbf{u}) \\
& =(k-1) R(z)\left(\prod_{\alpha=1}^{\ell-1} \oint_{\Gamma} \frac{d u_{\alpha}}{2 \pi \mathrm{i}} R\left(u_{\alpha}\right)\left(u_{\alpha}-z\right)\right) \Delta_{\ell-1}^{2}\left(u_{1}, \ldots, u_{\ell-1}\right) S\left(z, u_{1}, \ldots, u_{\ell-1}\right) \\
& \quad+\prod_{\alpha=1}^{k-1} \oint_{\Gamma} \frac{d u_{\alpha} R\left(u_{\alpha}\right)}{2 \pi \mathrm{i}\left(u_{\alpha}-z\right)} \prod_{\alpha=k}^{\ell} \oint_{\Gamma \cup z} \frac{d u_{\alpha} R\left(u_{\alpha}\right)}{2 \pi \mathrm{i}\left(u_{\alpha}-z\right)} \Delta_{\ell}^{2}(\mathbf{u}) S(\mathbf{u}) .
\end{aligned}
$$


In particular for $k=\ell+1$,

$$
\begin{aligned}
& \left(\prod_{\alpha=1}^{\ell} \oint_{\Gamma \cup z} \frac{d u_{\alpha} R\left(u_{\alpha}\right)}{2 \pi \mathrm{i}\left(u_{\alpha}-z\right)}\right) \Delta_{\ell}^{2}(\mathbf{u}) S(\mathbf{u})-\left(\prod_{\alpha=1}^{\ell} \oint_{\Gamma} \frac{d u_{\alpha} R\left(u_{\alpha}\right)}{2 \pi \mathrm{i}\left(u_{\alpha}-z\right)}\right) \Delta_{\ell}^{2}(\mathbf{u}) S(\mathbf{u}) \\
& \quad=\ell R(z)\left(\prod_{\alpha=1}^{\ell-1} \oint_{\Gamma} \frac{d u_{\alpha}}{2 \pi \mathrm{i}} R\left(u_{\alpha}\right)\left(u_{\alpha}-z\right)\right) \Delta_{\ell-1}^{2}\left(u_{1}, \ldots, u_{\ell-1}\right) S\left(z, u_{1}, \ldots, u_{\ell-1}\right) .
\end{aligned}
$$

Proof of Lemma 8.2. The identity (110) is obviously true for $k=1$. We now proceed by induction: let it be true for fixed $k \geq 1$; then we show its truth for $k+1$. In the second expression on the right hand side of equation 110 one computes the residue at $u_{k}=z$, yielding

$$
\begin{aligned}
& \prod_{\alpha=1}^{k-1} \oint_{\Gamma} \frac{d u_{\alpha} R\left(u_{\alpha}\right)}{2 \pi \mathrm{i}\left(u_{\alpha}-z\right)} \prod_{\alpha=k}^{\ell} \oint_{\Gamma \cup z} \frac{d u_{\alpha} R\left(u_{\alpha}\right)}{2 \pi \mathrm{i}\left(u_{\alpha}-z\right)} \Delta_{\ell}^{2}(\mathbf{u}) S(\mathbf{u}) \\
& =\prod_{\alpha=1}^{k-1} \oint_{\Gamma} \frac{d u_{\alpha} R\left(u_{\alpha}\right)}{2 \pi \mathrm{i}\left(u_{\alpha}-z\right)} \oint_{\Gamma \cup z} \frac{d u_{k} R\left(u_{k}\right)}{2 \pi \mathrm{i}\left(u_{k}-z\right)} \prod_{\alpha=k+1}^{\ell} \oint_{\Gamma \cup z} \frac{d u_{\alpha} R\left(u_{\alpha}\right)}{2 \pi \mathrm{i}\left(u_{\alpha}-z\right)} \Delta_{\ell}^{2}(\mathbf{u}) S(\mathbf{u}) \\
& =\prod_{\alpha=1}^{k} \oint_{\Gamma} \frac{d u_{\alpha} R\left(u_{\alpha}\right)}{2 \pi \mathrm{i}\left(u_{\alpha}-z\right)} \prod_{\alpha=k+1}^{\ell} \oint_{\Gamma \cup z} \frac{d u_{\alpha} R\left(u_{\alpha}\right)}{2 \pi \mathrm{i}\left(u_{\alpha}-z\right)} \Delta_{\ell}^{2}(\mathbf{u}) S(\mathbf{u}) \\
& +\prod_{\alpha=1}^{k-1} \oint_{\Gamma} \frac{d u_{\alpha} R\left(u_{\alpha}\right)}{2 \pi \mathrm{i}\left(u_{\alpha}-z\right)}\left(R(z) \prod_{\alpha=k+1}^{\ell} \oint_{\Gamma \cup z} \frac{d u_{\alpha} R\left(u_{\alpha}\right)}{2 \pi \mathrm{i}\left(u_{\alpha}-z\right)}\left(\Delta_{\ell}^{2} S\right)\left(u_{1}, \ldots, \begin{array}{c}
k \\
z
\end{array}, \ldots, u_{\ell}\right)\right) \\
& =\prod_{\alpha=1}^{k} \oint_{\Gamma} \frac{d u_{\alpha} R\left(u_{\alpha}\right)}{2 \pi \mathrm{i}\left(u_{\alpha}-z\right)} \prod_{\alpha=k+1}^{\ell} \oint_{\Gamma \cup z} \frac{d u_{\alpha} R\left(u_{\alpha}\right)}{2 \pi \mathrm{i}\left(u_{\alpha}-z\right)} \Delta_{\ell}^{2}(\mathbf{u}) S(\mathbf{u}) \\
& +R(z) \prod_{\alpha=1}^{k-1} \oint_{\Gamma} \frac{d u_{\alpha} R\left(u_{\alpha}\right)}{2 \pi \mathrm{i}}\left(\prod_{\alpha=k+1}^{\ell} \oint_{\Gamma \cup z} \frac{d u_{\alpha} R\left(u_{\alpha}\right)}{2 \pi \mathrm{i}}\right)
\end{aligned}
$$

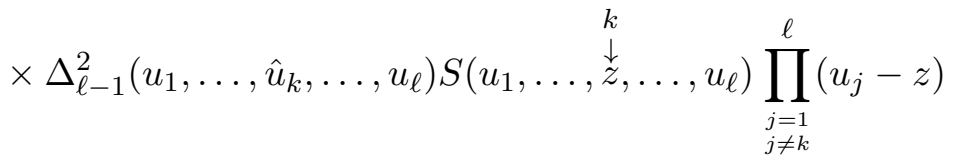

$$
\begin{aligned}
& =\prod_{\alpha=1}^{k} \oint_{\Gamma} \frac{d u_{\alpha} R\left(u_{\alpha}\right)}{2 \pi \mathrm{i}\left(u_{\alpha}-z\right)} \prod_{\alpha=k+1}^{\ell} \oint_{\Gamma \cup z} \frac{d u_{\alpha} R\left(u_{\alpha}\right)}{2 \pi \mathrm{i}\left(u_{\alpha}-z\right)} \Delta_{\ell}^{2}(\mathbf{u}) S(\mathbf{u}) \\
& +R(z) \prod_{\alpha=1}^{\ell-1} \oint_{\Gamma} \frac{d u_{\alpha}}{2 \pi \mathrm{i}} R\left(u_{\alpha}\right)\left(u_{\alpha}-z\right) \Delta_{\ell-1}^{2}\left(u_{1}, \ldots, u_{\ell-1}\right) S\left(z, u_{1}, \ldots, u_{\ell-1}\right) .
\end{aligned}
$$


The latter is obtained by renaming the variables $\left(u_{1}, \ldots, u_{k-1}, u_{k+1}, \ldots, u_{\ell}\right) \rightarrow$ $\left(u_{1}, \ldots, u_{\ell-1}\right)$ and upon noticing that in the second expression the integration over $\Gamma \cup z$ can be replaced by $\Gamma$, since the integrand has no residue at $z$. One also uses the symmetry of the function $S$. This ends the proof of Lemma 8.2.

Proof of Proposition 8.1: First expression in (109). The starting point is expression (95) for the kernel $\mathbb{K}^{\text {red }}$, and in particular its $\mathbb{K}_{2}$-piece. Use the expression 51 for $\widetilde{\Omega}_{\mathcal{R}}^{(1)}(v, w)$ in 96$)$, combined with expression 53 in Corollary 3.3 and (51), leading to:

$$
\begin{aligned}
d \oint_{\Gamma_{0}} & \frac{d w \widetilde{\Omega}_{\mathcal{R}}^{(1)}(v, w)}{2 \pi \mathrm{i} w^{y+1}(1-w)^{n}} \\
& =d\left(\prod_{\alpha=2}^{d} \oint_{\Gamma(\mathcal{R})} \frac{d u_{\alpha}}{2 \pi \mathrm{i} Q\left(u_{\alpha}\right)}\right) \Delta_{d}\left(v, u_{2}, \ldots, u_{d}\right) \widetilde{\Delta}_{d, n}^{\left(\mathbf{y}_{\mathrm{cut}}\right)}\left(y, u_{2}, \ldots, u_{d}\right) \\
& =d\left(\oint_{\Gamma(y, y-1, \ldots, y+n-N)}-\oint_{\Gamma_{\tau}}\right) \frac{(N-n) ! P(z) d z}{2 \pi \mathrm{i}(z-y)_{N-n+1}} \widetilde{\Omega}_{\mathcal{R}}(v, z),
\end{aligned}
$$

in terms of $\widetilde{\Omega}_{\mathcal{R}}(v, z)$, as in 107 . This establishes the first formula 109 .

Second expression in (109). The following identities holds:

$$
\frac{\Omega_{\mathcal{R}}(v, z)+d(z-v) \frac{P(z)}{Q(z)} \widetilde{\Omega}_{\mathcal{R}}(v, z)}{\Omega_{\mathcal{R}}(0,0)}=\frac{\Omega_{\mathcal{L}}(v, z)}{\Omega_{\mathcal{L}}(0,0)} \text { and } \frac{\widetilde{\Omega}_{\mathcal{R}}(v, z)}{\Omega_{\mathcal{R}}(0,0)}=-\frac{\widetilde{\Omega}_{\mathcal{L}}(v, z)}{\Omega_{\mathcal{L}}(0,0)} \text {. }
$$

It suffices to show that

$$
\begin{aligned}
(-1)^{d} \Omega_{\mathcal{L}}(v, z) & =\Omega_{\mathcal{R}}(v, z)+d(z-v) \frac{P(z)}{Q(z)} \widetilde{\Omega}_{\mathcal{R}}(v, z) \\
(-1)^{d-1} \widetilde{\Omega}_{\mathcal{L}}(v, z) & =\widetilde{\Omega}_{\mathcal{R}}(v, z) .
\end{aligned}
$$

Remembering the degrees of the polynomials $P$ and $Q$ in (21), we have that each integrand in the $d$-fold integral $\Omega_{\mathcal{L}}(v, z)$ and in the $d-1$-fold integral $\widetilde{\Omega}_{\mathcal{L}}(v, z)$ (as in Proposition 8.1 have degree (in a fixed $u_{\alpha}$ ) equal to

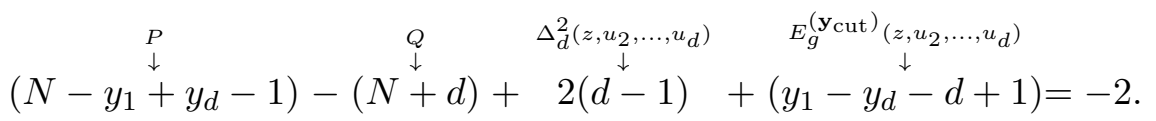

So the integrands have no pole at $\infty$. Thus the contour $-\Gamma(\mathcal{L})$ in $\Omega_{\mathcal{L}}$ (as in (113) ) can be deformed to a contour $\Gamma(\mathcal{R} \cup z)$; so we have $\left(C_{N, d}\right.$ is the constant in Proposition 3.1

$$
(-1)^{d} \frac{\Omega_{\mathcal{L}}(v, z)}{C_{N, d}}=\left(\prod_{\alpha=1}^{d} \oint_{\Gamma(\mathcal{R} \cup z)} \frac{d u_{\alpha} P\left(u_{\alpha}\right)}{2 \pi \mathrm{i} Q\left(u_{\alpha}\right)} \frac{u_{\alpha}-v}{u_{\alpha}-z}\right) E_{g}^{\left(\mathbf{y}_{\mathrm{cut}}\right)}(u) \Delta_{d}(u)^{2},
$$


which has the form of Lemma 8.2 , with $\ell=d$ and $R(u)=\frac{P(u)}{Q(u)}(u-v), S=E_{g}^{\left(\mathbf{y}_{\text {cut }}\right)}$, modulo a sign of $(-1)^{d}$. Then the right hand side of 114 equals, by formula 111) and 107,

$$
\begin{aligned}
\frac{\Omega_{\mathcal{R}}(v, z)}{C_{N, d}}+d(z-v) \frac{P(z)}{Q(z)} \prod_{2}^{d} \oint_{\Gamma(\mathcal{R})} \frac{d u_{\alpha} P\left(u_{\alpha}\right)}{2 \pi \mathrm{i} Q\left(u_{\alpha}\right)}\left(v-u_{\alpha}\right)\left(z-u_{\alpha}\right) \\
\quad \times \Delta_{d-1}^{2}\left(u_{2}, \ldots, u_{d}\right) E_{g}^{\left(\mathbf{y}_{\mathrm{cut}}\right)}\left(z, u_{2}, \ldots, u_{d}\right) \\
=\frac{1}{C_{N, d}}\left(\Omega_{\mathcal{R}}(v, z)+d(z-v) \frac{P(z)}{Q(z)} \widetilde{\Omega}_{\mathcal{R}}(v, z)\right),
\end{aligned}
$$

establishing the first identity (114). The second identity (113) is simpler, since the integrands have no pole about $u_{\alpha}=z$. Therefore the contour about $\mathcal{L}$ can be deformed to a contour about $\mathcal{R}$ in each of the $d-1$ integrals. This shows both identities 113$)$, upon using $\Omega_{\mathcal{L}}(0,0)=(-1)^{d} \Omega_{\mathcal{R}}(0,0)$, ending the proof of Proposition 8.1 .

Proof of Theorem 1.1. It suffices to prove:

$$
\frac{\Omega_{\mathcal{L}}(v, z)}{\Omega_{\mathcal{L}}(0,0)}=\frac{Q_{\mathcal{L}}(v)}{Q_{\mathcal{L}}(z)} \frac{\Omega_{r}^{+}(v, z)}{\Omega_{r}^{+}(0,0)}, \quad d \frac{\widetilde{\Omega}_{\mathcal{L}}(v, z)}{\Omega_{\mathcal{L}}(0,0)}=\frac{Q_{\mathcal{L}}(v) Q_{\mathcal{L}}(z)}{r+1} \frac{\Omega_{r+1}^{-}(v, z)}{\Omega_{r}^{+}(0,0)},
$$

$\Omega_{\mathcal{L}}, \widetilde{\Omega}_{\mathcal{L}}$ were defined in 108 , and $\Omega_{r}^{+}$and $\Omega_{r+1}^{-}$in 8 .

The proof will depend on the two identities below (116) and (117). Set $J:=$ $\left\{i_{1}<\cdots<i_{k}\right.$, with $\left.x_{i_{\alpha}} \in \mathcal{L}\right\}$, viewed as particles and the corresponding set of holes $j_{1}<\cdots<j_{\ell}$, also with $x_{j_{\alpha}} \in \mathcal{L}$ and $|\mathcal{L}|=k+\ell$. Let $\varphi(u)$ be a rational function with no roots or poles along $\mathcal{L}$. For the ease of notation set momentarily $\Delta(\mathcal{L}):=\left\{\right.$ vandermonde determinant of variables $x_{i}$ in $\left.\mathcal{L}\right\}$. Then

$$
\prod_{\alpha=1}^{k} \frac{\varphi\left(x_{i_{\alpha}}\right)}{Q_{\mathcal{L}}^{\prime}\left(x_{i_{\alpha}}\right)}=\prod_{x_{i} \in \mathcal{L}} \frac{\varphi\left(x_{i}\right)}{Q_{\mathcal{L}}^{\prime}\left(x_{i}\right)} \prod_{\alpha=1}^{\ell} \frac{Q_{\mathcal{L}}^{\prime}\left(x_{j_{\alpha}}\right)}{\varphi\left(x_{j_{\alpha}}\right)} .
$$

In formula (54) of Lemma 3.4. set $n=k, m=\ell$ and $n+m=k+\ell=|\mathcal{L}|$, and $\mathbf{x}=\left(x_{i_{1}}, \ldots, x_{i_{k}}\right)$ and $\mathbf{x}^{c}=\left(x_{j_{1}}, \ldots, x_{j_{\ell}}\right)$, and $Q:=Q_{\mathcal{L}}(z)$, leading to:

$$
\Delta_{k}(\mathbf{x})= \pm \frac{\Delta(\mathcal{L}) \Delta_{\ell}\left(\mathbf{x}^{c}\right)}{\prod_{\alpha=1}^{\ell} Q_{\mathcal{L}}^{\prime}\left(x_{j_{\alpha}}\right)} .
$$

Then, combining the three facts (116), (117) and (44) above, we have for $k, \ell \geq 0$ 
such that $k+\ell=|\mathcal{L}|$, with $\mathbf{x}$ and $\mathbf{x}^{c}$ as above:

$$
\begin{aligned}
& \left(\prod_{\alpha=1}^{k} \oint_{\Gamma(\mathcal{L})} \frac{d u_{\alpha} \varphi\left(u_{\alpha}\right)}{2 \pi \mathrm{i} Q_{\mathcal{L}}\left(u_{\alpha}\right)}\right)\left(S \Delta_{k}^{2}\right)(u)=\sum_{\substack{i_{1}<\cdots<i_{k} \\
x_{i_{\alpha}} \in \mathcal{L}}} k !\left(S \Delta_{k}^{2}\right)(\mathbf{x})\left(\prod_{\alpha=1}^{k} \frac{\varphi\left(x_{i_{\alpha}}\right)}{Q_{\mathcal{L}}^{\prime}\left(x_{i_{\alpha}}\right)}\right) \\
& =\frac{k !}{\ell !} \Delta^{2}(\mathcal{L}) \prod_{x_{i} \in \mathcal{L}} \frac{\varphi\left(x_{i}\right)}{Q_{\mathcal{L}}^{\prime}\left(x_{i}\right)} \ell ! \sum_{\substack{j_{1}<\cdots<j_{\ell} \\
x_{j_{\alpha}} \in \mathcal{L}}}\left(\prod_{\alpha=1}^{\ell} \frac{Q_{\mathcal{L}}^{\prime}\left(x_{j_{\alpha}}\right)}{\varphi\left(x_{j_{\alpha}}\right)\left(Q_{\mathcal{L}}^{\prime}\left(x_{j_{\alpha}}\right)\right)^{2}}\right)\left(\widetilde{S} \Delta_{\ell}^{2}\right)\left(\mathbf{x}^{c}\right) \\
& =\frac{k !}{\ell !} \Delta^{2}(\mathcal{L})\left(\prod_{x_{i} \in \mathcal{L}} \frac{\varphi\left(x_{i}\right)}{Q_{\mathcal{L}}^{\prime}\left(x_{i}\right)}\right)\left(\prod_{\alpha=1}^{\ell} \oint_{\Gamma(\mathcal{L})} \frac{d u_{\alpha}}{2 \pi \mathrm{i} \varphi\left(u_{\alpha}\right) Q_{\mathcal{L}}\left(u_{\alpha}\right)}\right)\left(\widetilde{S} \Delta_{\ell}^{2}\right)(u) .
\end{aligned}
$$

We now apply the formula above to two different $k, \ell$ and $\varphi(u)$ (themselves depending on $v, z)$ :

$$
\begin{aligned}
& \varphi(u):=\frac{P(u)(v-u)}{Q_{\mathcal{R}}(u) Q_{\mathfrak{C}}(u)(z-u)} \quad \varphi(u):=\frac{P(u)(v-u)(z-u)}{Q_{\mathcal{R}}(u) Q_{\mathfrak{C}}(u)} \\
& S\left(u_{1}, \ldots, u_{d}\right)=E_{g}^{\left(\mathbf{y}_{\text {cut }}\right)}\left(u_{1}, \ldots, u_{d}\right) \quad \text { or } \quad S\left(z, u_{2}, \ldots, u_{d}\right)=E_{g}^{\left(\mathbf{y}_{\text {cut }}\right)}\left(z ; u_{2}, \ldots, u_{d}\right) \\
& \widetilde{S}\left(u_{1}, \ldots, u_{r}\right)=\widetilde{E}_{g}^{\left(\mathbf{y}_{\text {cut }}\right)}\left(u_{1}, \ldots, u_{r}\right) \quad \widetilde{S}\left(z, u_{1}, \ldots, u_{r+1}\right)=\widetilde{E}_{g}^{\left(\mathbf{y}_{\text {cut }}\right)}\left(z ; u_{1}, \ldots, u_{r+1}\right) \\
& k=d, \ell=r \quad k=d-1, \quad \ell=r+1 .
\end{aligned}
$$

Te map $S \mapsto \widetilde{S}$ above refers to the operation on symmetric functions explained in (44). Using the expression (7) for $h(u)$, and remembering the definition s $^{13}$ (107), (108) and (8) of $\Omega_{\mathcal{L}}$ and $\Omega_{r}$, the first substitution 1119 in 118 leads to the following expression, where $C_{N, d}$ is the constant in $(42)$ and where $C_{\mathcal{L}}$ is a constant 14 depending on the geometry of $\mathbf{P}$ only:

$$
\begin{aligned}
\frac{\Omega_{\mathcal{L}}(v, z)}{C_{N, d} C_{N, d}^{\prime}} & =\left(\prod_{\alpha=1}^{d} \oint_{\Gamma(\mathcal{L})} \frac{d u_{\alpha} P\left(u_{\alpha}\right)}{2 \pi \mathrm{i} Q\left(u_{\alpha}\right)} \frac{v-u_{\alpha}}{z-u_{\alpha}}\right)\left(E_{g}^{\left(\mathbf{y}_{\text {cut }}\right)} \Delta_{d}^{2}\right)(u) \\
& =\frac{d !}{r !} C_{\mathcal{L}} \frac{Q_{\mathcal{L}}(v)}{Q_{\mathcal{L}}(z)}\left(\prod_{\alpha=1}^{r} \oint_{\Gamma(\mathcal{L})} \frac{d u_{\alpha} h\left(u_{\alpha}\right)}{2 \pi \mathrm{i}} \frac{z-u_{\alpha}}{v-u_{\alpha}}\right)\left(\widetilde{E}_{g}^{\left(\mathbf{y}_{\mathrm{cut}}\right)} \Delta_{r}^{2}\right)\left(u_{1}, \ldots, u_{r}\right) \\
& =\frac{d !}{r !} C_{\mathcal{L}} \frac{Q_{\mathcal{L}}(v)}{Q_{\mathcal{L}}(z)} \Omega_{r}^{+}(v, z),
\end{aligned}
$$

whereas the second substitution (119) in 1118 yields, remembering the definitions

\footnotetext{
${ }^{13}$ as in Proposition 8.1 .

${ }^{14}$ To be precise, $C_{\mathcal{L}}=\Delta(\mathcal{L})^{2} \prod_{x_{i} \in \mathcal{L}} \frac{P\left(x_{i}\right)}{Q_{\mathcal{L}}^{\prime}\left(x_{i}\right) Q_{\mathcal{R}}\left(x_{i}\right) Q_{\mathcal{C}}\left(x_{i}\right)}=\Delta(\mathcal{L})^{2} \prod_{x_{i} \in \mathcal{L}} \frac{P\left(x_{i}\right)}{Q^{\prime}\left(x_{i}\right)}$.
} 
107, 108 and 8 of $\widetilde{\Omega}_{\mathcal{L}}$ and $\widetilde{\Omega}_{r+1}$

$$
\begin{aligned}
\frac{\widetilde{\Omega}_{\mathcal{L}}(v, z)}{C_{N, d}}= & \left(\prod_{\alpha=2}^{d} \oint_{\Gamma(\mathcal{L})} \frac{d u_{\alpha} P\left(u_{\alpha}\right)}{2 \pi \mathrm{i} Q\left(u_{\alpha}\right)}\left(v-u_{\alpha}\right)\left(z-u_{\alpha}\right)\right) \\
& =\frac{(d-1) !}{(r+1) !} C_{\mathcal{L}}^{\left(\mathbf{y}_{\mathrm{cut}}\right)}\left(z ; u_{2}, \ldots, u_{d}\right) \Delta_{d-1}^{2}(v) Q_{\mathcal{L}}(z)\left(\prod_{\alpha=1}^{r+1} \oint_{\Gamma(\mathcal{L})} \frac{d u_{\alpha} h\left(u_{\alpha}\right)}{2 \pi \mathrm{i}\left(v-u_{\alpha}\right)\left(z-u_{d}\right)}\right) \\
& \widetilde{E}_{g}^{\left(\mathbf{y}_{\mathrm{cut}}\right)}\left(z ; u_{1}, \ldots, u_{r+1}\right) \Delta_{r+1}^{2}\left(u_{1}, \ldots, u_{r+1}\right) \\
= & \frac{(d-1) !}{(r+1) !} C_{\mathcal{L}} Q_{\mathcal{L}}(v) Q_{\mathcal{L}}(z) \Omega_{r+1}^{-}(v, z) .
\end{aligned}
$$

These two identities lead at once to the ratios (115). Substituting these ratios in the kernel 109 establishes Theorem 1.1.

Corollary 8.3 For $|\mathcal{L}|=d$, we have that

$$
\frac{\Omega_{\mathcal{L}}(v, z)}{\Omega_{\mathcal{L}}(0,0)}=\frac{Q_{\mathcal{L}}(v)}{Q_{\mathcal{L}}(z)}
$$

Proof of Corollary 8.3: Here $r=0$ and thus $\Omega_{r}(v, z)=1$ and the corollary follows from 115.

\section{References}

[1] Mark Adler, Sunil Chhita, Kurt Johansson and Pierre van Moerbeke: Tacnode GUE-minor processes and double Aztec diamonds, Probab. Theory Related Fields 162, no. 1-2, 275-325 (2015)

[2] Mark Adler, Kurt Johansson and Pierre van Moerbeke: Double Aztec diamonds and the tacnode process. Adv. Math. 252 (2014), 518-571.

[3] Mark Adler, Kurt Johansson and Pierre van Moerbeke: Lozenge tilings of hexagons with cuts and asymptotic fluctuations. To appear.

[4] Mark Adler and Pierre van Moerbeke: Coupled GUE-minor Processes. Intern Math Research Notices, 21 (2015) (arXiv:1312.3859)

[5] Dan Betea, J. Bouttier, P. Nejjar and M. Vuletic: The free boundary Schur process and applications. (arXiv:1704.05809) 
[6] A. Bufetov and A. Knizel: Asymptotics of random domino tilings of rectangular Aztec diamonds (arXiv: 1604.01491)

[7] Alexei Borodin, Patrik L. Ferrari, Michael Prähofer, Tomohiro Sasamoto: Fluctuation properties of the TASEP with periodic initial configuration J. Stat. Phys. 129 (2007) (arXiv:math-ph/0608056)

[8] A. Borodin, V. Gorin and E. M. Rains, q-Distributions on boxed plane partitions, Selecta Math. 16 (2010), 731-789.

[9] Alexei Borodin, Eric M. Rains: Eynard-Mehta theorem, Schur process, and their Pfaffian analogs J. Stat. Phys. 121 (2005), no. 3-4, 291-317. (arXiv:math-ph/0409059)

[10] Alexei Borodin: Determinantal point processes, The Oxford handbook of random matrix theory, 231249, Oxford Univ. Press, Oxford, 2011.

[11] Mihai Ciucu and Ilse Fischer Lozenge tilings of hexagons with arbitrary dents. Adv. in Appl. Math. 73, 1-22. (2016)

[12] H. Cohn, M. Larsen and J. Propp. The shape of a typical boxed plane partition, The New York Journal of Mathematics. 4 137-165 (1998)

[13] M. Defosseux: Orbit measures, random matrix theory and interlaced determinantal processes, Ann. Inst. H. Poincar Probab. Statist. 46, 209-249. (2010)

[14] Erik Duse, Kurt Johansson, Anthony Metcalfe The Cusp-Airy Process (arXiv:1510.02057)

[15] Erik Duse and Anthony Metcalfe: Asymptotic geometry of discrete interlaced patterns: Part I. Internat. J. Math. 26 (2015), 1550093.

[16] Erik Duse and Anthony Metcalfe: Asymptotic geometry of discrete interlaced patterns: Part II. (arXiv:1507.00467)

[17] Kurt Johansson, Discrete orthogonal polynomial ensembles and the Plancherel measure, Ann. of Math. 153, 259296. (2001)

[18] Kurt Johansson, Non-intersecting paths, random tilings and random matrices, Probab. Theory Related Fields 123 (2002), 225-280.

[19] Kurt Johansson, Discrete polynuclear growth and determinantal processes, Comm. Math. Phys. 242 (2003), 277-329. 
[20] Kurt Johansson, Non-intersecting, simple, symmetric random walks and the extended Hahn kernel, Ann. Inst. Fourier (Grenoble) 55, 2129-2145. (2005)

[21] Kurt Johansson, The arctic circle boundary and the Airy process, Ann. Probab. 33, 130. (2005)

[22] Kurt Johansson: Edge Fluctuations og Limit Shapes, Harvard Lectures (Nov. 2016) (arXiv:1704.06035)

[23] Kurt Johansson and Eric Nordenstam: Eigenvalues of GUE minors, Electron. J. Probab. 11, 13421371 (2006).

[24] Vadim E. Gorin: Nonintersecting paths and the Hahn orthogonal polynomial ensemble, Funct. Anal. Appl. 42, 180197 (2008).

[25] Vadim E. Gorin: Bulk universality for random lozenge tilings near straight boundaries and for tensor products, to appear in Communications in Mathematical Physics. (arXiv:1603.02707)

[26] Pieter W. Kasteleyn: Graph theory and crystal physics. Graph Theory and Theoretical Physics pp. 43110 Academic Press, London (1967).

[27] Richard Kenyon: Lectures on dimers Statistical mechanics, 191-230, IAS/Park City Math. Ser., 16, Amer. Math. Soc., Providence, RI, 2009. arXiv: 0910.3129

[28] Richard Kenyon and Andrei Okounkov: Limit shapes and the complex Burgers equation, Acta Math. 199, no. 2, 263-302 (2007)

[29] Christian Krattenthaler: Advanced determinantal calculus, Séminaire Lotharingien Combin. 42 (1999) (The Andrews Festschrift), paper B42q, $67 \mathrm{pp}$

[30] Christian Krattenthaler, Descending plane partitions and rhombus tilings of a hexagon with a triangular hole, European J. Combin. 27 no. 7, 1138-1146 (2006)

[31] I. Macdonald. Symmetric functions and Hall polynomials. Oxford Mathematical Monographs. (1995).

[32] P. A. MacMahon, Memoir on the theory of the partition of numbersPart $V$. Partitions in two-dimensional space, Phil. Trans. R. S., 1911, A.

[33] Anthony Metcalfe: Universality properties of GelfandTsetlin patterns, Probab. Theory Related Fields 155(1-2) 303-346 (2013). 
[34] Jonathan Novak, Lozenge tilings and Hurwitz numbers, Journal of Stat. Phys., 161 , 509-517 (2015) (arXiv:math/0309074)

[35] Andrei Okounkov and Nicolai Reshetikhin: Correlation function of Schur process with application to local geometry of a random 3-dimensional Young diagram J. of the American Math. Society 16, 581-603 (2003)

[36] Andrei Okounkov and Nicolai Reshetikhin: The birth of a random matrix Mosc. Math. J. 6 , 553-566, 588.(2006)

[37] Leonid Petrov: Asymptotics of uniformly random lozenge tilings of polygons. Gaussian free field, Ann. Probab. 43143 (2015).

[38] Leonid Petrov: Asymptotics of random lozenge tilings via Gelfand-Tsetlin schemes. Probability Theory and Related Fields. 160, 3-4 (2014), 429-487. 